\title{
\begin{tabular}{l|l} 
Mibraries & DSpace@MIT
\end{tabular}
}

\author{
MIT Open Access Articles
}

Development of a set of $C \cdot G-$ to-G $\bullet C$ transversion base editors from CRISPRi screens, target-library analysis, and machine learning

The MIT Faculty has made this article openly available. Please share how this access benefits you. Your story matters.

Citation: Koblan, Luke W. et al. "Development of a set of $C \bullet G-t o-G \bullet C$ transversion base editors from CRISPRi screens, target-library analysis, and machine learning." Nature Biotechnology (June 2021): dx.doi.org/10.1038/s41587-021-00938-z. (c) 2021 The Author(s)

As Published: http://dx.doi.org/10.1038/s41587-021-00938-z

Publisher: Springer Science and Business Media LLC

Persistent URL: https://hdl.handle.net/1721.1/131196

Version: Author's final manuscript: final author's manuscript post peer review, without publisher's formatting or copy editing

Terms of use: Creative Commons Attribution-Noncommercial-Share Alike 


\section{Editorial Summary:}

Transversion base editing is enhanced through CRISPRi screens, target-library analysis, and machine learning.

\section{Title:}

\section{Development of a set of $C \cdot G-t o-G \cdot C$ transversion base editors from CRISPRi screens, target-library analysis, and machine learning}

Luke W. Koblan ${ }^{1,2,3, \dagger}$, Mandana Arbab ${ }^{1,2,3, \dagger}$, Max W. Shen ${ }^{1,2,3,4, \dagger}$, Jeffrey A. Hussmann ${ }^{5-9}$, Andrew V. Anzalone ${ }^{1,2,3}$, Jordan L. Doman ${ }^{1,2,3}$, Gregory A. Newby ${ }^{1,2,3}$, Dian Yang ${ }^{5,7-9}$, Beverly Mok $^{1,2,3}$, Joseph M. Replogle ${ }^{5,7-11}$, Albert Xu ${ }^{5,7,10,12}$, Tyler A. Sisley ${ }^{2}$, Jonathan S.

Weissman $^{5,7-9,9,10 *}$, Britt Adamson ${ }^{5,7,13,14 *}$, David R. Liu ${ }^{1,2,3 *}$

\footnotetext{
${ }^{1}$ Merkin Institute of Transformative Technologies in Healthcare, Broad Institute of Harvard and MIT, Cambridge, MA, USA.

${ }^{2}$ Department of Chemistry and Chemical Biology, Harvard University, Cambridge, MA, USA.

${ }^{3}$ Howard Hughes Medical Institute, Harvard University, Cambridge, MA, USA.

${ }^{4}$ Computational and Systems Biology Program, Massachusetts Institute of Technology, Cambridge, MA, USA.

${ }^{5}$ Department of Cellular and Molecular Pharmacology, University of California, San Francisco, San Francisco, CA 94158, USA

${ }^{6}$ Department of Microbiology and Immunology, University of California, San Francisco, San Francisco, CA 94158, USA

${ }^{7}$ Howard Hughes Medical Institute, University of California, San Francisco, San Francisco, CA 94158, USA

${ }^{8}$ Present address: Whitehead Institute for Biomedical Research, Cambridge, MA, USA.

${ }^{9}$ Present address: Department of Biology, Massachusetts Institute of Technology, Cambridge, MA, USA.

${ }^{10}$ Medical Scientist Training Program, University of California, San Francisco, San Francisco, CA 94158, USA

${ }^{11}$ Tetrad Graduate Program, University of California, San Francisco, San Francisco, CA, USA

${ }^{12}$ Biomedical Sciences Graduate Program, University of California, San Francisco, San Francisco, CA, USA

${ }^{13}$ Lewis-Sigler Institute for Integrative Genomics, Princeton University, Princeton, NJ 08544, USA

${ }^{14}$ Department of Molecular Biology, Princeton University, Princeton, NJ 08544, USA
}

\section{${ }^{\dagger}$ Denotes equal contribution}

*Correspondence should be addressed to Jonathan Weissman (weissman@wi.mit.edu), Britt Adamson (badamson@princeton.edu), and David R. Liu (drliu@fas.harvard.edu) 
Programmable C·G-to-G・C base editors (CGBEs) have broad scientific and therapeutic potential, but their editing outcomes have been difficult to predict and their editing efficiency and product purity are often low. We describe a suite of engineered CGBEs paired with machine learning models to enable efficient, high-purity C-G-to$G \cdot C$ base editing. We performed a CRISPRi screen targeting DNA repair genes to identify factors that affect $C \cdot G-$ to-G.C editing outcomes and used these insights to develop CGBEs with diverse editing profiles. We characterized ten promising CGBEs on a library of 10,638 genomically integrated target sites in mammalian cells and trained machine learning models that accurately predict the purity and yield of editing outcomes $(R=0.90)$ using these data. These CGBEs enable correction to the wild-type coding sequence of $\mathbf{5 4 6}$ disease-related transversion single-nucleotide variants with $>90 \%$ precision (mean $96 \%$ ) and up to $70 \%$ efficiency (mean $14 \%$ ). Computational prediction of optimal CGBE-sgRNA pairs enables high-purity transversion base editing at $>4$-fold more target sites than can be achieved using any single CGBE variant. 
Single-nucleotide variants (SNVs) represent approximately half of currently known human pathogenic gene variants ${ }^{1}$. Base editors, fusions of programmable DNA-binding proteins with base-modifying enzymes, enable conversion of individual target nucleotides in the genome ${ }^{2-10}$. The two major classes of base editors are cytosine base editors (CBEs), which convert $C \cdot G$ to $T \cdot A$, and adenine base editors $(A B E s)$, which convert $A \cdot T$ to $G \cdot C^{2,3,8}$. CBEs and ABEs can install transition mutations with high efficiency and product purity (the fraction of all edited alleles that contain only the desired edit), but in general, cannot efficiently install transversion mutations including $C \cdot G$ to $G \cdot C^{2,5,11,12}$.

We previously demonstrated that CBE editing byproducts, including $\mathrm{C} \cdot \mathrm{G}-\mathrm{to}-\mathrm{G} \cdot \mathrm{C}$ or $\mathrm{C} \cdot \mathrm{G}-\mathrm{to}-\mathrm{A} \cdot \mathrm{T}$ transversion outcomes, are inhibited by knockout of cellular uracil DNA Nglycosylase (UNG) or by fusion of uracil glycosylase inhibitor (UGI) $2,7,8,11,12$, suggesting that transversion byproducts result from an abasic intermediate that is generated by UNGcatalyzed excision of deaminated target cytosines (Fig. 1a). Consistent with this model, firstgeneration $\mathrm{C} \cdot \mathrm{G}-\mathrm{to}-\mathrm{G} \cdot \mathrm{C}$ base editors (CGBEs) were CBE derivatives that lack UGI domains ${ }^{11}$. These CGBEs, including editors with fusions to UNG and other DNA-repair proteins ${ }^{13-16}$, can provide efficient $C \cdot G-$ to- $G \cdot C$ editing but only at a minority of tested target sites with few criteria to identify sites amenable to CGBE editing ${ }^{13-15}$.

Previously, we used libraries containing thousands of genomically integrated target sites and corresponding guide RNAs in mammalian cells to comprehensively characterize $\mathrm{CBE}$ and $\mathrm{ABE}$ base editing profiles. We used these data to train machine learning models (collectively named $B E-H i v e$ ) that learned the sequence determinants driving $\mathrm{CBE}$ and $\mathrm{ABE}$ base editing outcomes ${ }^{12,17}$. We envisioned that broad characterization of the sequence determinants of CGBE editing outcomes could enable accurate prediction of editing efficiencies and product purities, and thus facilitate the broader use of CGBEs.

Here, we performed a focused CRISPR interference (CRISPRi) screen to identify DNA repair genes that impact cytosine base editing efficiency and purity. Guided by these data, we constructed various fusions proteins containing deaminases and Cas proteins fused to DNA repair components to engineer novel CGBEs with promising $C \cdot G-$ to-G $\cdot C$ editing activities. We characterized ten such CGBEs with diverse editing profiles using a "comprehensive context library" of 10,638 genomically integrated, highly variable target sites in mouse embryonic stem cells (mESCs) ${ }^{12}$. We used the resulting data to train machine learning models that successfully predict CGBE editing efficiency, purity, and bystander editing patterns with high accuracy (CGBE-Hive), enabling reliable identification of CGBE variants and target sites that 
together support high-purity $\mathrm{C} \cdot \mathrm{G}-\mathrm{to}-\mathrm{G} \cdot \mathrm{C}$ editing. Moreover, we show that editing activity is predicted with substantially higher accuracy by deep learning models compared to simpler models, indicating that CGBE-Hive has learned complex sequence features that play important roles in determining C-to-G editing activity. Notably, 247 cytosines predicted by CGBE-Hive to be edited by a CGBE with $>80 \% \mathrm{C} \cdot \mathrm{G}-\mathrm{to}-\mathrm{G} \cdot \mathrm{C}$ editing purity were indeed edited in mammalian cell experiments with an average of $83 \%$ purity.

The panel of CGBEs in this study offer diverse editing profiles that collectively expand the sequence landscape amenable to high-quality $C \cdot G$-to-G.C editing by up to 4.1 -fold over the number predicted to be amenable to editing by any single CGBE. Finally, we demonstrate CGBE-mediated correction of 546 disease-associated single-nucleotide variants (SNVs) with $>90 \%$ precision among the resulting edited amino acid sequences. These findings advance our understanding of transversion base editing outcomes and provide new CGBEs that improve the scope and utility of base editing.

\section{Results}

Exploring the activity of DNA glycosylases in $C \cdot G-t o-G \cdot C$ transversion outcomes

Previous work suggested that excision of uracil from genomic DNA to generate an abasic lesion followed by error-prone polymerase activity on the strand opposite the abasic site results in C•G-to-G・C and C.G-to-A•T transversion outcomes (Fig. 1a) ${ }^{2,11,16}$. Motivated by this model, we sought to develop $\mathrm{C} \cdot \mathrm{G}-\mathrm{to}-\mathrm{G} \cdot \mathrm{C}$ base editors that enhanced uracil excision at CBE-edited nucleotides. We started with a CBE architecture lacking UGI (BE4B) (bpNLSAPOBEC1-Cas9 D10A-bpNLS; abbreviated AC), similar to other reported CGBEs ${ }^{13-15}$.

We fused a variety of known uracil excising and binding enzymes to the $\mathrm{C}$-terminus of the $B E 4 B(A C)$ scaffold and assessed the frequency of $C \cdot G-$ to- $G \cdot C$ edits across five genomic loci in HEK293T cells (Fig. 1b). Several glycosylases (i.e., SMUG1, MBD4, and TDG2) did not alter editing outcomes, and fusion to UNG led to a reduction of $\mathrm{C} \cdot \mathrm{G}-\mathrm{to}-\mathrm{G} \cdot \mathrm{C}$ editing yield and purity at three out of five targeted sites, consistent with a recent report ${ }^{13}$. Nevertheless, we found that fusion of a UNG orthologue from Mycobacterium smegmatis (UdgX) moderately improved $\mathrm{C} \cdot \mathrm{G}$-to-G.C product purity by 1.2 -fold on average ${ }^{18-20}$, with the largest improvement at the RNF2 locus $(56 \pm 0.8 \%$ with $\mathrm{BE} 4 \mathrm{~B}$ to $72 \pm 2.1 \%$ with $\mathrm{AC}-\mathrm{UdgX} ; \mathrm{p}=0.0002$, Student's two-sided t-test) and significant changes observed at HEK site 2 C6, HEK site 3 C5, and EMX1 C6 ( $p<0.01$, Student's two-sided t-test). However, we observed only modest changes to editing yield (1.1-fold relative to BE4B at the most efficiently edited $C$ across the 
five tested genomic loci). These observations suggested that fusion partners may enhance $\mathrm{C} \cdot \mathrm{G}-\mathrm{to}-\mathrm{G} \cdot \mathrm{C}$ transversion base editing outcomes.

Next, we asked whether the orientation of the glycosylase fusion impacts editing outcomes. We constructed BE4B (AC) fusion variants with either UdgX (abbreviated X) or GFP in three orientations: at either the $\mathrm{N}$ - or C-terminus (e.g., XAC or ACX) or between the deaminase and Cas9 (e.g., AXC). We observed that C.G-to-G•C editing was similar or slightly improved for UdgX fusions compared to $\mathrm{N}$ - and C-terminal GFP fusions (Fig. 1c). However, the editing efficiency and purity of AXC was modestly higher than that of the best GFP fusion at a majority of sites (four out of five sites for efficiency; three out of five sites for purity). We chose to advance the AXC architecture since it offered similar or better performance than the XAC and ACX variants at these test loci.

CRISPRi screen for determinants of base editing outcomes

Next, we investigated whether other DNA repair or translesion synthesis factors impact C.G-to-G.C editing outcomes of $A X C$. We observed no significant changes in editing purity of AXC in individual UNG, APE1/APEX1, MLH1, REV1 knockout cell lines, and direct AXC fusions to mammalian polymerase domains did not consistently improve editing outcomes (Supplementary Figs. 1-2; Supplementary Discussion 1). We thus performed a much broader search for modulators of cytosine transversion editing by performing two highthroughput genetic screens.

Using a recently developed screening platform ${ }^{21}$ capable of reading out DNA repair outcomes by DNA sequencing (Fig. 2a-b, Supplementary Fig. 3a), we investigated how knockdown of each of 476 genes, a set enriched for regulators of DNA repair, impacts the activity of BE1 (deaminase-dCas9) and BE4B (AC) editors. Briefly, we transduced an sgRNA library (1,513 gene-targeting sgRNAs and 60 non-targeting controls, Supplementary Table 1) into HeLa cells stably expressing the CRISPRi effector dSpCas9-KRAB ${ }^{22}$. After allowing 5 days for gene knockdown, we transfected the cells with plasmids encoding SaCas9-based CBEs (either SaCas9-BE1 or SaCas9-BE4B) and an SaCas9 sgRNA that targets a sequence adjacent to the genomically integrated SpCas9 sgRNA sequences. Notably, we used SaCas9-based CBEs to avoid guide RNA exchange between the base editors and CRISPRi machinery. A key aspect of this approach was that the proximity of the target site and CRISPRi sgRNA enabled these features to be read out together by paired-end DNA sequencing, thus linking editing outcomes to CRISPRi perturbation identities (Fig. 2a). To 
prepare samples for sequencing, we isolated genomic DNA from treated cells, affixed unique molecular identifiers (UMIs) to DNA fragments containing both the sgRNA expression cassettes and edited target sites, and sequenced the linked sgRNA, target sites, and UMI sequences. Comparing frequencies of editing outcomes from each CRISPRi sgRNA with those from non-targeting sgRNAs (examples in Fig. 2b, Supplementary Fig. 3a) then identified genes that promote or suppress various editing outcomes (Supplementary Table 2).

Consistent baseline activity of BE1 and BE4B in the screens enabled quantitation of editing differences driven by CRISPRi sgRNAs (Fig. 2, Supplementary Fig. 3, Supplementary Fig. 4). To evaluate differences in point mutations, we calculated the effects of all CRISPRi sgRNAs on the frequencies of two major categories: outcomes containing any C.G-to-T•A point mutation and outcomes containing any $\mathrm{C} \cdot \mathrm{G}-\mathrm{to}-\mathrm{G} \cdot \mathrm{C}$ point mutation (Fig. $\mathbf{2 c}$ ). For both classes, the effects of individual CRISPRi sgRNAs were consistent between replicates (Fig. 2c, upper left and lower right panels). Comparison between classes though revealed that some CRISPRi sgRNAs showed different effects on C•G-to-T•A versus C•G-to$\mathrm{G} \cdot \mathrm{C}$ outcomes (Fig. 2c, upper right panel), indicating that specific genes influence partitioning between these outcomes. In the BE4B screen, the clearest differential effects resulted from sgRNAs targeting UNG (Fig. 2b, c). Consistent with the effects of UGI fusions and UNG loss ${ }^{2,11}$, UNG knockdown increased frequencies of C•G-to-T•A editing while decreasing frequencies of $C \cdot G-t o-G \cdot C$ editing. Notably, the effects of $U N G$ repression on BE1 editing were not as significant or straightforward (Supplementary Fig. 3a,c), perhaps reflecting differences in how nicked versus unnicked target substrates are processed (Fig. 2b and Supplementary Fig. 3a).

One advantage to screening with sequencing-based readouts was that we could detect changes to a diverse range of editing products. For example, we also observed that CRISPRi-mediated depletion of double-strand breaks (DSB) repair genes affect the frequency of rare indels caused by base editing, though these pathway-phenotype relationships were not always straightforward (Supplementary Fig. 4a, Supplementary Table 2). Indeed, while knockdown of HDR factors BRCA1, BRCA2, and PALB2 increased AC-generated deletions, depletion of the HDR gene BLM decreased them. Interestingly, depletion of $B R C A 2$ was also among the strongest reducers of $\mathrm{C} \cdot \mathrm{G}$-to-T•A editing outcomes (Supplementary Fig. 4b). We also identified genes that affect the base editing window (Supplementary Figs. 4c, 5; Supplementary Discussion 2). 
To identify genes that specifically promoted $\mathrm{C} \cdot \mathrm{G}-\mathrm{to}-\mathrm{G} \cdot \mathrm{C}$ editing, we calculated the relative fraction of outcomes containing any $\mathrm{C} \cdot \mathrm{G}-\mathrm{to}-\mathrm{G} \cdot \mathrm{C}$ edit among outcomes containing any point mutation for each CRISPRi sgRNA (Fig. 2d and Supplementary Fig. 4d). The gene whose knockdown most significantly reduced the $\mathrm{C} \cdot \mathrm{G}-\mathrm{to}-\mathrm{G} \cdot \mathrm{C}$ editing fraction compared to non-targeting sgRNAs was RFWD3, an E3 ligase with multiple roles in DNA repair recently identified as required for successful translesion synthesis across a variety of genomic lesions $^{23}$. Other hits included UNG; multiple subunits of the replicative polymerase POLD and replicative clamp loader RFC; EXO1; translesion polymerases REV1 and REV3L; and $R A D 18$, an E3 ubiquitin ligase involved in translesion synthesis (Supplementary Table 2). The different phenotypes for REV1 knockdown versus our individual knockout cell line may arise from compensatory mechanisms that could alter DNA repair outcomes in cells lacking $R E V 1$. We also identified genes whose knockdown reduced frequencies of both $\mathrm{C} \cdot \mathrm{G}-\mathrm{to}-\mathrm{T} \cdot \mathrm{A}$ and $\mathrm{C} \cdot \mathrm{G}-$ to-G.C base editing for both BE1 and BE4B (Supplementary Fig. 4e), including ASCC3, which may act by affecting accessibility of the target locus, a known determinant of base editing efficiency ${ }^{2,3,8}$. Together, these screen results suggest important roles for DNA replication processes, especially translesion synthesis, in modulating $\mathrm{C} \cdot \mathrm{G}$-to-G・C base editing outcomes.

\section{CBE fusion proteins can alter $C \cdot G$-to-G・C transversion outcomes}

To further advance the development of CGBEs, we generated new CGBE candidates by fusing AXC, the prototype CGBE described above, to proteins nominated by our CRISPRi screens. These included those encoded by genes that reduced $\mathrm{C} \cdot \mathrm{G}-\mathrm{to}-\mathrm{G} \cdot \mathrm{C}$ editing following knockdown, including DDX1, EXO1, POLD1, POLD2, POLD3, RAD18, RBMX, REV1, RFWD3, and TIMELESS, and several additional genes involved in DNA polymerization, some of which also affected editing outcomes in the CRISPRi screen (PCNA, POLH, POLK, UBE2I, and UBE2T, Supplementary Table 2).

We fused each of these proteins to the $\mathrm{N}$ - or $\mathrm{C}$-terminus of $\mathrm{AXC}$ to assess their effect on $\mathrm{C} \cdot \mathrm{G}$-to-G.C editing efficiency or purity and assessed their editing performance at five genomic loci in HEK293T cells. Three proteins increased $\mathrm{C} \cdot \mathrm{G}-\mathrm{to}-\mathrm{G} \cdot \mathrm{C}$ editing purity when fused to the N-terminus of AXC (Supplementary Fig. 6a): DNA polymerase D2 (POLD2), exonuclease 1 (EXO1), and RNA binding motif protein X-linked (RBMX). Editing improvements for fused constructs varied by site. The most pronounced effects were observed at the RNF2 locus, where editing purity significantly improved from $54 \pm 1.4 \%$ with 
AXC to $73 \pm 0.4 \%$ with RBMX-AXC, $74 \pm 1.4 \%$ for EXO1-AXC, and $77 \pm 0.8 \%$ for POLD2-AXC $(p<0.001$, Student's two-sided t-test). Marginal improvements in purity were also observed at HEK site 2, HEK site 3, and HEK site 4 loci. At RNF2 we also observed a significant increase in editing yield from $43 \pm 2.4 \%$ with $A X C$ to $50 \pm 5.2 \%$ with RBMX-AXC, $53 \pm 3.6 \%$ with EXO1-AXC, and 55 $\pm 5.5 \%$ for POLD2-AXC ( $p<0.05$, Student's two-sided t-test). C-terminal fusions typically did not perform as well as $\mathrm{N}$-terminal fusions (Supplementary Data 1).

Encouraged by these improvements, we developed additional candidate CGBEs containing RBMX, EXO1, POLD2, and UdgX as fusions to AXC. We compared single and dual pairwise fusion architectures for these components, testing $\mathrm{N}$ - and $\mathrm{C}$-terminal dual fusions as well as tandem $\mathrm{N}$ terminal fusions $(\mathrm{N}-, \mathrm{N}-)$ using 32-residue linkers identified in a linker-testing experiment for these constructs (Supplementary Fig. 7). From a total of 28 single- and dual-fusion proteins tested, the four dual fusion architectures POLD2deaminase-UdgX-nCas9-RBMX, POLD2-deaminase-UdgX-nCas9-UdgX, UdgXdeaminase-UdgX-nCas9-UdgX, and UdgX-deaminase-UdgX-nCas9-RBMX further increased $C \cdot G-$ to-G.C editor yield and purity at some sites (on average, by $+10 \%$ and $+13 \%$, respectively) compared to single fusion architectures across nine cytosines in five genomic loci (Supplementary Fig. 6b).

Collectively, these results indicate that CGBEs, including fusions to proteins identified in the CRISPRi screen, can affect C•G-to-G・C editing outcomes in a site-dependent manner. Some base editing applications may prioritize protein size over other base editing characteristics. We therefore explored the use of trans-splicing split-inteins as a means to reduce the size of large CGBEs into two smaller protein components ${ }^{24}$, and observed no changes in editing outcomes of split-CGBEs compared to their full-length counterparts (Supplementary Fig. 8). When necessary, these split CGBE variants may support favorable cytosine transversion outcomes without requiring the expression of full-length proteins.

\section{Base editor deaminase and Cas9 domains bias repair outcomes}

We next sought to understand how different deaminase domains affect $C \cdot G-$ to-G・C editing in the AXC architecture. Since the base editing window may influence cytosine transversion outcomes ${ }^{2,11,12}$, we examined a panel of catalytically impaired deaminases that support different $\mathrm{CBE}$ editing windows ${ }^{25}$ and observed an increase in C.G-to-G.C editing purity at three of five tested loci (Fig. 3a). The APOBEC1 R126E R132E (EE) ${ }^{25}$ deaminase showed the greatest improvement, averaging 1.2-fold higher product purity at HEK site 2, 
HEK site 3, and RNF2. Editing yield with these deaminase alternatives varied by locus. We observed similar or reduced editing yield compared to $A X C$ at four out of five loci that is likely due to the lower catalytic activity of these deaminases, though reduced yield did not correlate with altered $\mathrm{C} \cdot \mathrm{G}-\mathrm{to}-\mathrm{G} \cdot \mathrm{C}$ purity. Editing yield by EE-AXC at the RNF2 locus significantly improved $(A X C=52 \pm 3.2 \%$ vs. EE-AXC $=66 \pm 3.5 \%, p=0.007$, Student's two-sided t-test).

We also hypothesized that changes to the Cas9 binding domain of CGBEs could alter editing windows and $\mathrm{C} \cdot \mathrm{G}$-to-G.C editing outcomes by altering the competition between Cas 9 and repair machinery for access to the target locus. We assessed AXC editors that use Cas 9 variants with different binding kinetics, including new variants with combinations of previously reported Cas9 mutations (Fig. 3 b) ${ }^{26-29}$. AX-HF-nCas9 substantially improved C $\cdot G-t o-G \cdot C$ editing at the $C 9$ position of the HEK site 3 locus, increasing yield $(A X C=34 \pm 1.9 \%$ vs. $A X-$ HF-nCas9 $=52 \pm 1.7 \%$, ) and purity ( $A X C=49 \pm 2.2 \%$ vs. AX-HF-nCas $9=60 \pm 1.2 \%)(p<0.005$ for both, Student's two-sided t-test) (Fig. 3b). AX-Hypa-nCas9 showed similar effects but AXHF-nCas9 typically performed modestly better. These results suggest Cas protein binding parameters can affect $C \cdot G-$ to-G.C editing yield and purity of CGBEs at some target loci.

The balance of editing yield and purity among candidate CGBEs and the variability in these two measures across different loci suggests that different target sites will be best edited by different CGBEs. Therefore, a suite of CGBEs with different kinetics and substrate preferences would likely enable efficient and high-purity $C \cdot G-$ to-G $\cdot C$ editing across a broader range of diverse target sequences than could be achieved by any single CGBE variant alone.

Combining deaminase, Cas9 domain, and DNA repair fusion proteins into new CGBEs We integrated the above findings from varying protein fusions, deaminases, and Cas domains into improved CGBEs. We evaluated the four most promising dual-fusion $A X C$ editors (POLD2-AXC-RBMX, POLD2-AXC-UdgX, UdgX-AXC-RBMX, and UdgX-AXCUdgX), four single-fusion AXC editors (POLD2-AXC, RBMX-AXC, EXO1-AXC, and UdgX$A X C), A X C s$ with deaminase variants of those same editors, and direct deaminase-nCas9 CGBEs without additional fusion proteins. The five cytidine deaminases tested in these 10 CGBE architectures included rAPOBEC1, EE, Anc689 (ancestrally-reconstructed APOBEC1 node $689^{30}$ ), eA3A, and eA3A-T31 $\mathrm{A}^{12}$. In addition, we tested both SpCas9 nickase and HFCas9 nickase variants. In total, we evaluated 95 candidate CGBEs at eight genomic loci in HEK293T cells. 
No single CGBE outperformed all other candidates at all sites (Fig. 4a). To identify a set of the most promising CGBEs, we selected 32 editors that demonstrated improved C.Gto-G.C editing outcomes at some sites for testing at eight additional genomic loci (Fig. 4b). We used these data to identify ten CGBEs with high purity, yield, and maximally distinct activities at different endogenous loci using quadratic programming and hierarchical clustering (Supplementary Methods): Anc689-nCas9, UdgX-Anc689-UdgX-nCas9-RBMX, eA3A-nCas9, RBMX-eA3A-UdgX-HF-nCas9, RBMX-eA3A-UdgX-nCas9, EE-nCas9, UdgX-EE-UdgX-nCas9-UdgX, APOBEC1-nCas9, UdgX-APOBEC1-UdgX-HF-nCas9, and POLD2-APOBEC1-UdgX-nCas9-UdgX.

To test how this set of CGBEs performed in human cell lines other than HEK293T cells, we assayed the ability of each of these CGBEs to edit five target genomic sites in K562, U2OS, and HeLa (Supplementary Fig. 9). We observed that while CGBE outcomes vary modestly by cell type, the top-performing CGBE variants for each tested site were generally the same in all three additional cell lines. These results indicate that deaminase, Cas protein, and DNA repair protein variants can improve $C \cdot G-t_{0}-G \cdot C$ editing in across different cell types.

\section{Target library characterization of CGBEs}

We observed that different target loci were best edited by different CGBEs, indicating that diverse CGBE sequence preferences may be strong determinants of $C \cdot G$-to-G.C editing efficiency and purity. Previously, we used high-throughput analysis of base editing outcomes at thousands of genomically integrated target sequences to better understand $C B E$ and $A B E$ sequence-activity relationships, and we used these data to train machine learning models that facilitate the selection of target sequences amenable to $\mathrm{C} \cdot \mathrm{G}$-to-G.C conversion by $\mathrm{CBEs}^{12}$. We envisioned that comprehensive characterization of our top ten promising and diverse CGBEs could similarly aid in the selection of targets amenable to efficient and highpurity $\mathrm{C} \cdot \mathrm{G}-$ to-G$\cdot \mathrm{C}$ editing by specific $\mathrm{CGBEs}$.

We characterized each of the ten CGBEs using a high-throughput genome-integrated library assay of 10,638 matched sgRNA and target pairs in mESCs, previously referred to as the "comprehensive context library"12. The target sequences in this library cover all possible sequence contexts surrounding the edited $C \cdot G$ with minimal sequence bias (Fig. 5a,

Supplementary Methods). To detect editing outcomes with high sensitivity, we maintained an average coverage of $\geq 300 x$ per library member throughout the course of the experiment and an average sequencing depth of $\geq 4,000 x$ per target. We collected two biological 
replicates per CGBE characterization experiment. We previously validated that the library assay data has strong consistency between biological replicates and is concordant with data from base editing endogenous genomic loci ${ }^{12,31}$.

We used the resulting library data to quantify editing windows and product purities for each CGBE (Fig. 5b, Supplementary Methods). CGBE editing activity was generally centered around protospacer position 6 with editing window widths ranging from $3 \mathrm{nt}$ (EEnCas9; positions 5-7) to $8 \mathrm{nt}$ (UdgX-APOBEC1-UdgX-HF-nCas9 nickase; positions 4-11). The editing windows of CGBEs with additional components beyond Cas and deaminase domains were shifted by up to 3 nt compared to direct deaminase-Cas fusions, indicating that CGBE protein fusions can affect editing window size and position.

Engineered CGBE architectures showed significant improvements in C.G-to-G•C product purity compared to simple deaminase-nCas 9 fusions. Across the 10,638 target sites in the comprehensive context library, the fusion CGBEs POLD2-APOBEC1-UdgX-nCas9UdgX, UdgX-EE-UdgX-nCas9-UdgX, and UdgX-Anc689-UdgX-nCas9-RBMX showed $25 \%$ higher mean $\mathrm{C} \cdot \mathrm{G}-\mathrm{to}-\mathrm{G} \cdot \mathrm{C}$ purity than their corresponding deaminase-nCas 9 counterparts within each editor's editing window $\left(P<5.1 \times 10^{-9}\right.$; Welch's t-test) (Fig. 5c). We observed large variation in CGBE editing efficiency, with mean efficiency ranging from $1.8 \%$ by UdgX-EE-UdgX-nCas9-UdgX to $23.0 \%$ by Anc689-nCas9 across the comprehensive context library within the same experimental batch. Notably, the protein fusion CGBEs exhibiting increased $C \cdot G-$ to-G.C purity also reduced editing yield by $1.4-$ to 1.6 -fold on average.

C.G-to-G.C editing purity exceeded $90 \%$ for at least one of the tested CGBEs at 895 cytosines across the comprehensive context library. Some cytosines edited with purities as high as $90-100 \%$ by some CGBEs were edited with purity as low as $0-10 \%$ by other CGBEs, indicating that these CGBEs indeed offer complementary editing characteristics, and confirming that a panel of diverse CGBEs maximizes the utility of $C \cdot G-$ to-G.C base editing compared to using any single CGBE (Fig. $\mathbf{5 d}$ ). We clustered CGBEs by C.G-to-G・C editing purity across the comprehensive context library and observed that engineered CGBEs did not cluster by deaminase (Fig. 5e), indicating that protein fusion engineering of CGBE architectures resulted in distinct sequence preferences governing $C \cdot G-t o-G \cdot C$ editing. 
C.G-to-G.C product purity of CGBEs varies substantially by sequence context (Fig. 5f). We observed $24.7 \pm 26.3 \%$ average $C \cdot G-$ to-G.C purity across all tested CGBEs for cytosines positioned near the center of the editing window, with substantial variation across target sequences: the top $5 \%$ had $>79.6 \%$ C.G-to-G.C purity while the bottom $5 \%$ had $<1.0 \%$. To decipher the sequence determinants that underly CGBE activity, we computed simple motifs for editing efficiency and transversion purity using a logistic regression model that considers each nucleotide independently (Fig. 5g, Supplementary Methods) ${ }^{12}$. These motifs revealed that $\mathrm{T} \underline{\mathrm{C}}$ is strongly favored while $\mathrm{G} \underline{\mathrm{C}}$ is disfavored for editing efficiency across the tested CGBEs. We further trained gradient-boosted regression trees to predict CGBE editing efficiency sequence context, which achieved good accuracy with $R=0.57-0.77$ at heldout target sites. Consistent with our previous characterization of BE4 variants ${ }^{12}$, we observed sequence motifs that associated RCTA with higher $C \cdot G-$ to-G.C purity $(R=A$ or $G)$ across all characterized CGBEs. Cytosines in an ACTA motif were edited with an average $\mathrm{C} \cdot \mathrm{G}-\mathrm{to}-\mathrm{G} \cdot \mathrm{C}$ purity of $68.7 \%(N=1,760)$ across CGBEs, substantially higher than the $24.7 \%$ average across all sequence contexts, indicating a major role for sequence context in determining C.G-to-G.C editing outcomes. These simple target sequence motifs predicted $27.0 \%-53.3 \%$ of the variation in $\mathrm{C} \cdot \mathrm{G}-\mathrm{to}-\mathrm{G} \cdot \mathrm{C}$ purity.

Next, we trained BE-Hive models for these ten CGBEs (termed CGBE-Hive) and evaluated the models' ability to predict $\mathrm{C} \cdot \mathrm{G}$-to-G・C editing purity at held-out sequence contexts not seen during training. These models explained $58.3 \%-76.3 \%$ of the variance in C.G-to-G.C purity in the held-out dataset, a substantial improvement over logistic regression described above (27.0\%-53.3\%) (Fig. 5h). This performance improvement highlights that while $C \cdot G-t o-G \cdot C$ purity can be predicted using a simple motif such as RETA that considers each nucleotide independently, higher-order interactions between nucleotides learned by deep neural networks substantially improve $C \cdot G-t o-G \cdot C$ editing purity predictions. Collectively, these observations establish that CGBE editing efficiency and purity can be accurately predicted by machine learning models.

To further investigate sequence determinants of CGBE editing outcomes, we calculated target sequence motifs for cytosines with the highest $C \cdot G-t o-G \cdot C$ efficiency for each CGBE (Supplementary Methods). While most CGBEs shared sequence preferences favoring T $\underline{C}$ for overall editing efficiency and RCTA for purity, different CGBEs had distinct motifs that correlated with C.G-to-G・C yield. POLD2-APOBEC1-UdgX-nCas9-UdgX

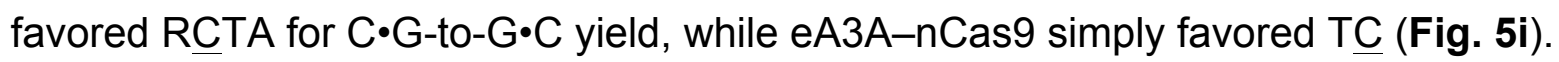


Interestingly, RBMX-eA3A-UdgX-nCas9 favored CTC, while UdgX-EE-UdgX-nCas9-UdgX favored TCT, and Anc689-nCas9 favored CTA (Fig. 5i). These observations reveal that different CGBEs show distinct sequence preferences that influence the yield of $C \cdot G$-to-G・C outcomes.

We provide machine learning models trained on up to 10,638 sgRNA-target pairs for these ten CGBEs in our online interactive web app (www.crisprbehive.design) ${ }^{12}$. Users can query sgRNAs and target sequences for data-driven predictions on editing outcomes of all CGBEs characterized in this study.

\section{Model-guided correction of pathogenic transversion SNVs}

To extend the applicability of these CGBEs, we assessed their compatibility with PAMvariant Cas9 proteins. We evaluated editing at eight loci by CGBEs using Cas9-NG, an engineered SpCas9 variant with broadened PAM compatibility ${ }^{32}$, and observed similar editing purities to SpCas9 CGBEs at NGG PAM substrates (Supplementary Fig. 10, 11). The best performing NG-CGBEs at each locus retained $>50 \%$ yield relative to SpCas 9 CGBEs at targets with NGG PAMs (Supplementary Fig. 10).

Given the broadened targeting scope of NG-CGBEs we sought to characterize their performance on the "transversion-enriched SNV library" ${ }^{12}$ in mESCs, which contains 3,400 sgRNA-target pairs selected by BE-Hive from 18,523 disease-related $G \cdot C$-to-C $\cdot G$ and A $\cdot T$-toC.G SNVs from the ClinVar and HGMD databases that are targetable by Cas9-NG ${ }^{1,33}$, predicted to be correctable by cytosine transversion base editing with high purity and yield. We generated the following NG-CGBEs based on their performance on the comprehensive context library: Anc689-nCas9-NG, APOBEC1-nCas9-NG, eA3A-nCas9-NG, UdgXAnc689-UdgX-nCas9-NG -RBMX, and UdgX-APOBEC1-UdgX-HF-nCas9-NG. As Cas9NG generally demonstrates reduced editing activity compared to wild-type SpCas $9^{32}$, similar to HF-Cas9, we included UdgX-APOBEC1-UdgX-nCas9-NG without the HF modifications as an alternative binding-impaired Cas9-fusion variant.

All six CGBEs tested on the transversion-enriched SNV library enabled high-purity C.G-to-G.C editing at disease-associated SNVs. At 247 cytosines predicted by CGBE-Hive to have $>80 \%$ C $\cdot G$-to-G.C editing purity, CGBEs demonstrated an average of $83 \%$ C.G-to-G・C editing purity (Fig. 6a). Each CGBE corrected $>200$ SNVs to their wild-type coding sequence with $>90 \%$ precision among edited amino acid sequences (amino acid correction precision; Fig. 6b), with a total of 546 unique SNVs across CGBEs. For example, in the genome- 
integrated library, eA3A-nCas9-NG corrected the G.C-to-C $\cdot G$ SNV in COL3A1 associated with Ehlers-Danlos syndrome ${ }^{34}$ with $71.4 \%$ yield and $92.8 \%$ purity, and corrected an SNV in BRCA2 associated with familial breast and ovarian cancer ${ }^{35}$ with $66.5 \%$ yield and $82.5 \%$ purity. The fusion CGBE UdgX-APOBEC1-UdgX-nCas9-NG corrected an SNV in NSD1 associated with Sotos syndrome ${ }^{36}$ with $40.0 \%$ yield and $73.4 \%$ purity and corrected an SNV in NIPBL associated with Cornelia de Lange syndrome ${ }^{37}$ with $38.8 \%$ yield and $76.9 \%$ purity. Collectively, these results reveal efficient and high-purity correction of hundreds of diseaserelated SNVs by CGBEs.

Notably, the UdgX-APOBEC1-UdgX-nCas9 CGBE maintained a similar high purity of C.G-to-G.C editing between HF-nCas9 and nCas9-NG variants. UdgX-APOBEC1-UdgXnCas9-NG, however, offered substantially better yield of genotype and coding sequence corrected G.C-to-C.G SNVs (Fig. 6a,b). These results suggest that fusion of CGBEs to Cas9-NG variants may obviate the need to use HF-variant Cas9-proteins to alter their binding kinetics to promote $\mathrm{C} \cdot \mathrm{G}-\mathrm{to}-\mathrm{G} \cdot \mathrm{C}$ editing outcomes.

The best-edited targets in the transversion-enriched SNV library varied greatly by CGBE. Some SNVs edited with $>90 \%$ purity by one CGBEs had purity below $5 \%$ for other CGBEs (Supplementary Fig. 12). CGBE-Hive models accurately accounted for this diversity in editing purity in the transversion-enriched SNV library, and accurately predicted the yield of exact genotype correction products and of alleles with corrected amino acid sequences ( $R=0.89-0.93$ and $R=0.91-0.94$, respectively, Fig. $6 \mathbf{c}$ ), as well as the DNA and amino acid correction precision ( $R=0.77-0.85$ and $R=0.82-0.90$, respectively, Fig. 6 d), including targets with multiple cytosines in the editing window. Since accurately predicting correction yield and precision requires accurate predictions for CGBE efficiency, $C \cdot G-$ to-G.C purity, and bystander editing patterns, these results establish that CGBE-Hive has learned important aspects of CGBE editing activity and can guide the use of CGBEs for high-purity correction of disease-related transversion SNVs.

Using CGBE-Hive to pick the best among the characterized CGBEs to correct each SNV should achieve greater $C \cdot G-$ to-G $\cdot C$ correction than applying any single CGBE to a set of targets. Indeed, we observed that using CGBE-Hive to choose the three CGBE variants predicted to best achieve the desired edit (top-3 performance) increased the number of targets corrected with $\geq 90 \%$ precision or to $\geq 40 \%$ efficiency by 4.1 - and 5.0 -fold, respectively, compared to the number of targets that are expected to be corrected with these precision and efficiency thresholds by picking any single CGBE (Fig. 6e). These improvements of 4.1- and 
5.0-fold by using the top three CGBE-Hive choices were nearly identical to the performance from picking the best CGBE out of all six options in hindsight. CGBE-Hive also displayed strong top-1 performance: Using CGBE-Hive to choose just a single CGBE increased the number of targets corrected with $\geq 90 \%$ precision or to $\geq 40 \%$ efficiency to 1.7 - and 4.0 -fold, respectively, compared to picking a single CGBE in expectation.

For correction precision, CGBE-Hive recovered the best performing CGBE variant in its top choice in $43.3 \%$ of targets and in its top three choices in $84.2 \%$ of target sequences. For correction yield, CGBE-Hive recovered the best-performing CGBE variant in its top choice in $67.5 \%$ of targets and in its top three choices in $97.2 \%$ of targets. These results collectively demonstrate that this panel of CGBEs have diverse editing activities that CGBEHive has learned to predict, to optimize selection of the most promising CGBE variant to use for a desired edit. These improvements were also observed at endogenous loci in HEK293T cells (Fig. 6f, Supplementary Discussion 3). Thus, CGBE-Hive enables researchers to reap the benefits of the diversity of CGBEs developed in this study without the need to test all CGBE variants.

Comparisons with recently reported CGBEs, prime editing, and off-target profiling

Next, we determined whether the CGBE variants described in this work extend the scope of $\mathrm{C} \cdot \mathrm{G}-\mathrm{to}-\mathrm{G} \cdot \mathrm{C}$ base editing beyond those accessible with recently described CGBEs or PE. We were encouraged to find that the CGBEs developed in this study extend the scope of C.G-to-G.C genome editing by enabling higher yields and product purities at a wider array of target sequences compared to the use of previously described CGBEs alone except at loci already edited with high yield and purity by deaminase-nCas9 constructs (Supplementary Fig. 13; Supplementary Discussion 4). Furthermore, we observed that these novel CGBEs complement prime editing (PE) technology ${ }^{38}$. We found PE typically offers higher product purities while editing with CGBEs offers higher editing yields at some loci (Supplementary Fig. 14; Supplementary Discussion 5), consistent with recent reports ${ }^{13-15,38}$. Notably, prime editing currently requires extensive optimization of pegRNA features to achieve highefficiency edits, while CGBE-Hive prediction obviates CGBE editor selection. CGBEs complement prime editing for efficient $C \cdot G-t o-G \cdot C$ editing, although additional optimization of both technologies may further improve their properties.

We also sought to characterize potential off-target editing outcomes of CGBEs. Since the genome-wide off-targets of base editors that use cytosine deaminase enzymes are 
known to be predominantly sgRNA dependent, we characterized Cas9-dependent off-target editing profiles of CGBEs by examining the activity of CGBEs at previously confirmed offtarget loci of corresponding Cas9:sgRNA complexes ${ }^{8}$. The architectural changes and protein fusions used to develop the CGBEs in this study resulted in lower Cas9-dependent off-target editing compared to corresponding CGBEs lacking protein fusions (Supplementary Fig. 11, 15), despite their generally higher on-target editing, perhaps because the more complex fusions or architectural changes introduce additional conformational requirements in editor:DNA complexes that are not met by some off-target loci (see Supplementary Discussion 6). While DNA repair protein CGBE components may result in additional Casindependent off-target effects, these are likely to differ by cell type and delivery method, and therefore are best assessed for each application.

\section{Discussion}

Understanding and controlling the outcomes of genome editing experiments are important challenges for achieving targeted, precise genome manipulation. We investigated molecular determinants of transversion base editing, including the effects of the deaminase and Cas effector domains, as well as many DNA repair proteins, and used these insights to engineer novel CGBEs. We characterized the editing outcomes and performance of these reagents using a high-throughput genome-integrated library assay in mammalian cells and identified sequence features that affect base editing outcomes of ten diverse CGBEs. We showed that C-to-G editing activity is predicted with substantially higher accuracy by deep learning models compared to simpler models, indicating that complex sequence features drive $\mathrm{C} \cdot \mathrm{G}$-to-G・C editing activity.

We provide trained CGBE-Hive machine learning models which accurately predict CGBE efficiency, $C \cdot G-t o-G \cdot C$ editing purity, and bystander editing patterns $(R=0.90)$ to enable predictable and consistently pure CGBE editing. We demonstrate a machine learning workflow using CGBE-Hive to identify optimal CGBE and sgRNA editing strategies to install a desired edit and show that this workflow expands high-efficiency and high-purity C.G-to-G・C editing to more loci than using any single CGBE by 5.0 -fold and 4.1 -fold with the top three CGBE-nominated choices. We demonstrate CGBE-mediated correction of the amino acid sequences of 546 disease-associated single nucleotide variants (SNVs) with $>90 \%$ precision. Furthermore, we demonstrated efficient and pure installation of four disease-relevant SNPs and tested the performance of these tools in other mammalian cell lines. Collectively, the 
base editor and computational tools presented in this work substantially improve the targeting scope, effectiveness, and utility of CGBE-mediated transversion base editing. 


\section{Data and code availability}

The target library sequencing data generated during this study are available at the NCBI Sequence Read Archive database under PRJNA631290. Data from the Repair-seq screens are available under PRJNA721212. Processed target library data used for training machine learning models have been deposited under the following DOls: 10.6084/m9.figshare.12275645 and 10.6084/m9.figshare.12275654.

\section{Code availability}

Code used for analyzing CRISPRi screens is available at https://github.com/jeffhussmann/repair-seq. Code used for target library data processing and analysis are available at https://github.com/maxwshen/lib-dataprocessing and https://github.com/maxwshen/lib-analysis. The machine learning models for CGBEs trained on target library data are available as a part of the BE-Hive interactive web application at https://crisprbehive.design and the BE-Hive Python package at https://github.com/maxwshen/be predict efficiency and https://github.com/maxwshen/be predict bystander.

\section{Acknowledgements}

This work was supported by U.S. NIH U01Al142756, UG3AI150551, RM1HG009490, R35GM118062, R35GM138167, P30CA072720, HHMI, and Princeton University. The authors acknowledge NSF Graduate Research Fellowships to L.W.K., M.W.S., and T.A.S.; a NWO Rubicon Fellowship to M.A.; a Jane Coffin Childs postdoctoral fellowship to A.V.A.; fellowship support from the NSF and Hertz Foundation to J.L.D.; a Helen Hay Whitney postdoctoral fellowship to G.A.N.; a Damon Runyon Postdoctoral Fellowship to D.Y.; a Singapore A*STAR NSS fellowship to B.M.; and NIH Ruth L. Kirschstein National Research Service Award F31NS115380 to J.M.R. J.A.H. was the Rebecca Ridley Kry Fellow of the Damon Runyon Cancer Research Foundation.

\section{Author contributions}

L.W.K, M.A., M.W.S., J.A.H., A.V.A., J.S.W., B.A., D.R.L. designed the research. L.W.K., M.A., M.W.S., J.A.H., A.V.A., J.L.D., G.A.N., D.Y., B.M., J.M.R., A.X., T.A.S., B.A. performed experiments. J.S.W., B.A., and D.R.L. supervised the project. L.W.K. and D.R.L. wrote the manuscript with input from all other authors.

\section{Author information}

The authors declare competing financial interests: J.A.H. is a consultant for Tessera Therapeutics. J.M.R. is a consultant for Maze Therapeutics. JSW is a consultant for and holds equity in Maze Therapeutics, Chroma Medicine, and KSQ Therapeutics. B.A. was a member of a ThinkLab Advisory Board for, and holds equity in, Celsius Therapeutics. D.R.L. is a consultant for, and holds equity in, Beam Therapeutics, Prime Medicine, Pairwise Plants, and Chroma Medicine. The remaining authors declare no competing interests. 


\section{References}

1. Landrum, M.J. et al. ClinVar: public archive of interpretations of clinically relevant variants. Nucleic Acids Res 44, D862-D868 (2016).

2. Komor, A.C., Kim, Y.B., Packer, M.S., Zuris, J.A. \& Liu, D.R. Programmable editing of a target base in genomic DNA without double-stranded DNA cleavage. Nature 533, 420-424 (2016).

3. Gaudelli, N.M. et al. Programmable base editing of A $\cdot T$ to $G \cdot C$ in genomic DNA without DNA cleavage. Nature 551, 464-471 (2017).

4. Gehrke, J.M. et al. An APOBEC3A-Cas9 base editor with minimized bystander and off-target activities. Nature Biotechnology 36, 977-982 (2018).

5. Nishida, K. et al. Targeted nucleotide editing using hybrid prokaryotic and vertebrate adaptive immune systems. Science 353, aaf8729-aaf8729 (2016).

6. Richter, M.F. et al. Phage-assisted evolution of an adenine base editor with improved Cas domain compatibility and activity. Nature Biotechnology 38, 883-891 (2020).

7. Rees, H.A. \& Liu, D.R. Base editing: precision chemistry on the genome and transcriptome of living cells. Nature Reviews Genetics 19, 770-788 (2018).

8. Anzalone, A.V., Koblan, L.W. \& Liu, D.R. Genome editing with CRISPR-Cas nucleases, base editors, transposases and prime editors. Nature Biotechnology 38, 824-844 (2020).

9. Gaudelli, N.M. et al. Directed evolution of adenine base editors with increased activity and therapeutic application. Nature Biotechnology 38, 892-900 (2020).

10. Mok, B.Y. et al. A bacterial cytidine deaminase toxin enables CRISPR-free mitochondrial base editing. Nature 583, 631-637 (2020).

11. Komor, A.C. et al. Improved base excision repair inhibition and bacteriophage $\mathrm{Mu}$ Gam protein yields C:G-to-T:A base editors with higher efficiency and product purity. Science Advances 3, eaao4774 (2017).

12. Arbab, M. et al. Determinants of Base Editing Outcomes from Target Library Analysis and Machine Learning. Cell 182, 463-480.e430 (2020).

13. Kurt, I.C. et al. CRISPR C-to-G base editors for inducing targeted DNA transversions in human cells. Nature Biotechnology 39, 41-46 (2020).

14. Zhao, D. et al. Glycosylase base editors enable C-to-A and C-to-G base changes. Nature Biotechnology 39, 35-40 (2020).

15. Chen, L. et al. Programmable C:G to G:C genome editing with CRISPR-Cas9-directed base excision repair proteins. Nature Communications 12 (2021).

16. Liu, D.R. \& Koblan, L.W. Cytosine to Guanine Base Editor. World Intellectual Property Organization (2018).

17. Marquart, K.F. et al. Predicting base editing outcomes with an attention-based deep learning algorithm trained on high-throughput target library screens. bioRxiv (2020).

18. Sang, P.B., Srinath, T., Patil, A.G., Woo, E.-J. \& Varshney, U. A unique uracil-DNA binding protein of the uracil DNA glycosylase superfamily. Nucleic Acids Res 43, 84528463 (2015).

19. Ahn, W.-C. et al. Covalent binding of uracil DNA glycosylase UdgX to abasic DNA upon uracil excision. Nat Chem Biol 15, 607-614 (2019).

20. Tu, J., Chen, R., Yang, Y., Cao, W. \& Xie, W. Suicide inactivation of the uracil DNA glycosylase UdgX by covalent complex formation. Nat Chem Biol 15, 615-622 (2019).

21. Hussmann, J.A. et al. Mapping the Genetic Landscape of DNA Double-strand Break Repair. BioRxiv (2021).

22. Gilbert, L.A. et al. CRISPR-mediated modular RNA-guided regulation of transcription in eukaryotes. Cell 154, 442-451 (2013). 
23. Gallina, I., Hendriks, I.A., Hoffmann, S., Larsen, N.B., Johansen, J., ColdingChristensen, C.S., Schubert, L., Sellés-Baiget, S., Fábián, Z., Kühbacher, U., Gao, A.O., Räschle, M., Rasmussen, S., Nielsen, M.L., Mailand, N., Duxin, J.P. The ubiquitin ligase RFWD3 is required for translesion DNA synthesis. Molecular Cell 81, 1-17 (2020).

24. Levy, J.M. et al. Cytosine and adenine base editing of the brain, liver, retina, heart and skeletal muscle of mice via adeno-associated viruses. Nat Biomed Eng 4, 97-110 (2020).

25. Kim, Y.B. et al. Increasing the genome-targeting scope and precision of base editing with engineered Cas9-cytidine deaminase fusions. Nature Biotechnology 35, 371-376 (2017).

26. Kleinstiver, B.P. et al. High-fidelity CRISPR-Cas9 nucleases with no detectable genome-wide off-target effects. Nature 529, 490-495 (2016).

27. Slaymaker, I.M. et al. Rationally engineered Cas9 nucleases with improved specificity. Science 351, 84-88 (2015).

28. Chen, J.S. et al. Enhanced proofreading governs CRISPR-Cas9 targeting accuracy. Nature 550, 407-410 (2017).

29. Lee, J.K. et al. Directed evolution of CRISPR-Cas9 to increase its specificity. Nature Communications 9, 3048 (2018).

30. Koblan, L.W. et al. Improving cytidine and adenine base editors by expression optimization and ancestral reconstruction. Nature Biotechnology 36, 843-846 (2018).

31. Shen, M.W. et al. Predictable and precise template-free CRISPR editing of pathogenic variants. Nature 563, 646-651 (2018).

32. Nishimasu, $\mathrm{H}$. et al. Engineered CRISPR-Cas9 nuclease with expanded targeting space. Science 361, 1259-1262 (2018).

33. Stenson, P.D. et al. Human Gene Mutation Database: towards a comprehensive central mutation database. Journal of Medical Genetics 45, 124-126 (2007).

34. Frank, M. et al. The type of variants at the COL3A1 gene associates with the phenotype and severity of vascular Ehlers-Danlos syndrome. European Journal of Human Genetics 23, 1657-1664 (2015).

35. Petrucelli, N., Daly, M.B. \& Feldman, G.L. Hereditary breast and ovarian cancer due to mutations in BRCA1 and BRCA2. Genetics in Medicine 12, 245-259 (2010).

36. Douglas, J. et al. NSD1 mutations are the major cause of Sotos syndrome and occur in some cases of Weaver syndrome but are rare in other overgrowth phenotypes. American journal of human genetics 72, 132-143 (2003).

37. Luna-Peláez, N. et al. The Cornelia de Lange Syndrome-associated factor NIPBL interacts with BRD4 ET domain for transcription control of a common set of genes. Cell Death Dis 10 (2019).

38. Anzalone, A.V. et al. Search-and-replace genome editing without double-strand breaks or donor DNA. Nature 576, 149-157 (2019).

39. Clement, K. et al. CRISPResso2 provides accurate and rapid genome editing sequence analysis. Nature Biotechnology 37, 224-226 (2019).

40. Horlbeck, M.A. et al. Compact and highly active next-generation libraries for CRISPRmediated gene repression and activation. eLife 5 (2016).

41. Gilbert, Luke A. et al. Genome-Scale CRISPR-Mediated Control of Gene Repression and Activation. Cell 159, 647-661 (2014).

42. Gilbert, Luke A. et al. CRISPR-Mediated Modular RNA-Guided Regulation of Transcription in Eukaryotes. Cell 154, 442-451 (2013). 
43. Sherwood, R.I. et al. Discovery of directional and nondirectional pioneer transcription factors by modeling DNase profile magnitude and shape. Nature Biotechnology $\mathbf{3 2}$, 171-178 (2014).

44. Paszke, A. et al. PyTorch: An Imperative Style, High-Performance Deep Learning Library. Advances in Neural Information Processing Systems 32, 8024-8035 (2019). 
a

mismatch or base excision repair

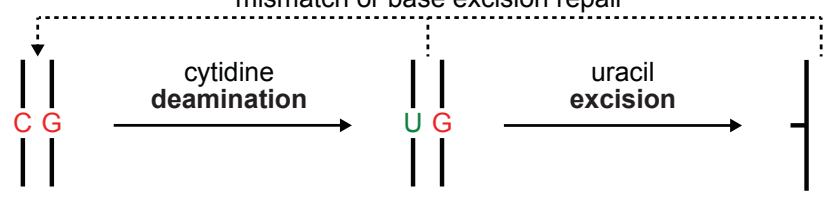

b
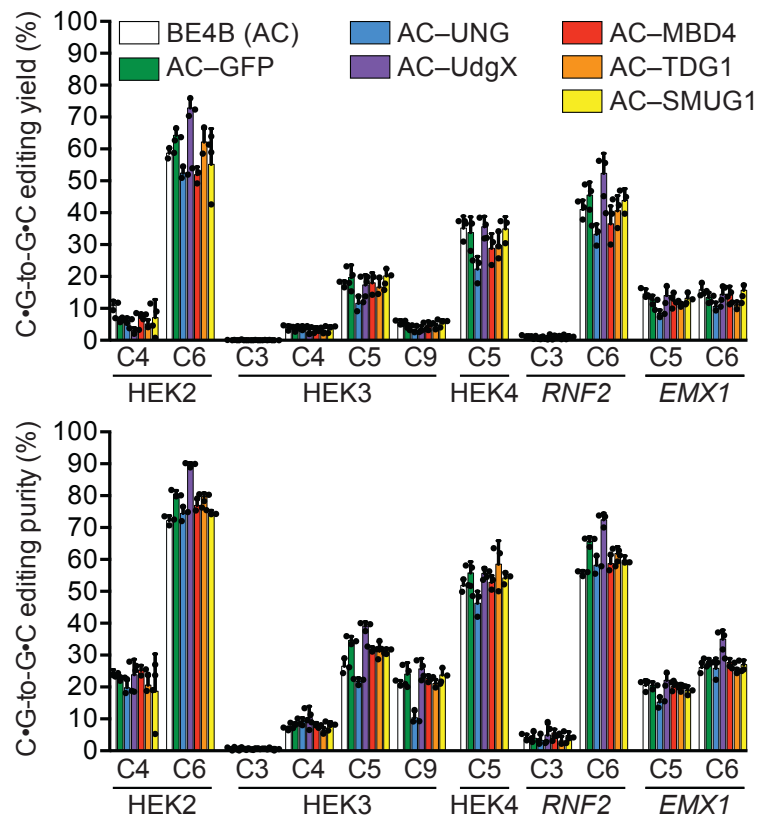

AC-Fusion:

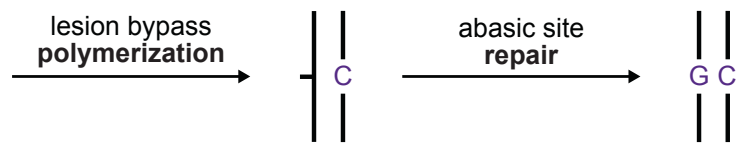

C
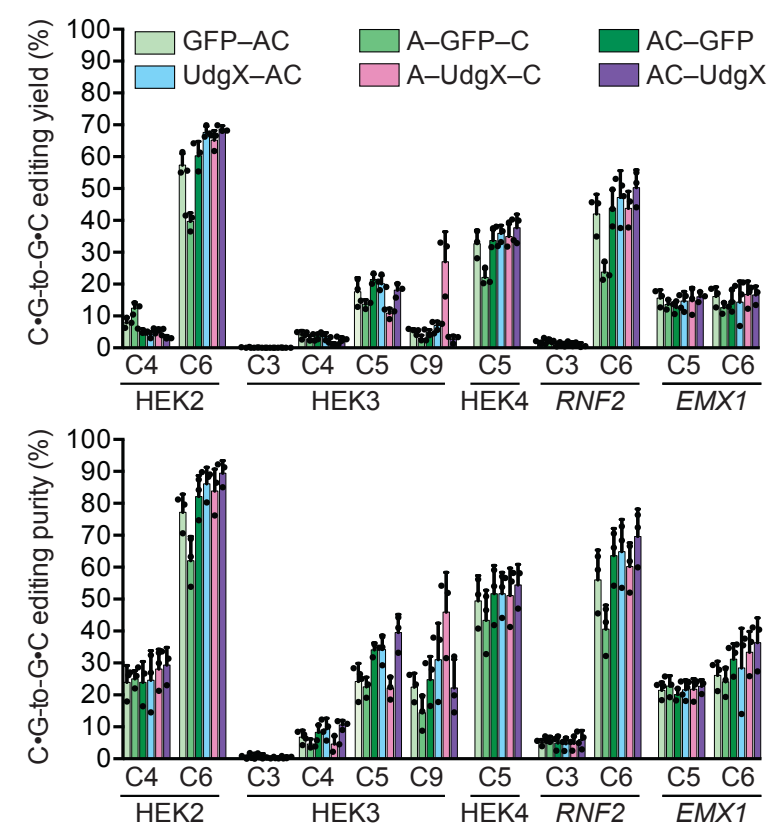

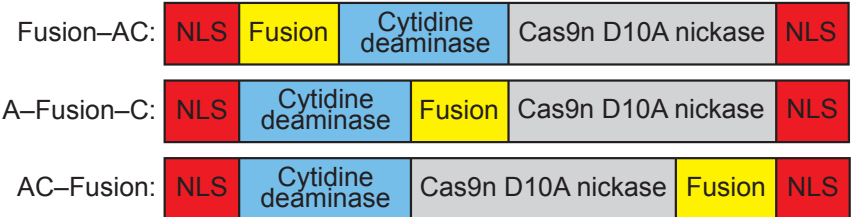

Figure 1. Development of prototype C.G-to-G・C base editors. (a) Potential pathway for C.G-to-G・C conversion. (b) C.G-to-G・C editing outcomes in HEK293T cells for C-terminal fusions of DNA glycosylases to BE4B (AC, APOBEC1 cytidine deaminase-Cas9 nickase). (c) Different fusion protein architectures lead to different $C \cdot G-t o-G \cdot C$ editing properties in HEK293T cells at the HEK3 locus for the Apo-UdgX-Cas9n (AXC) architecture. Values and error bars reflect the mean and standard deviation of three biological replicates, shown as individual data points. HEK2=HEK site 2; HEK3=HEK site 3; HEK4=HEK site 4. C4, C6, and similar annotations indicate the in-window target nucleotides where the SpCas9 PAM is at positions 21-23. 
a

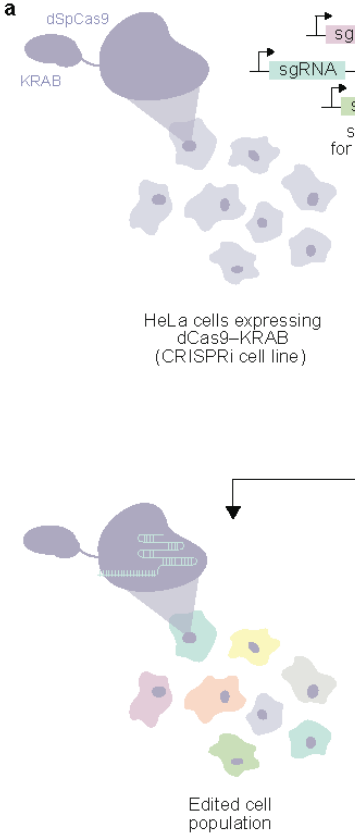

b

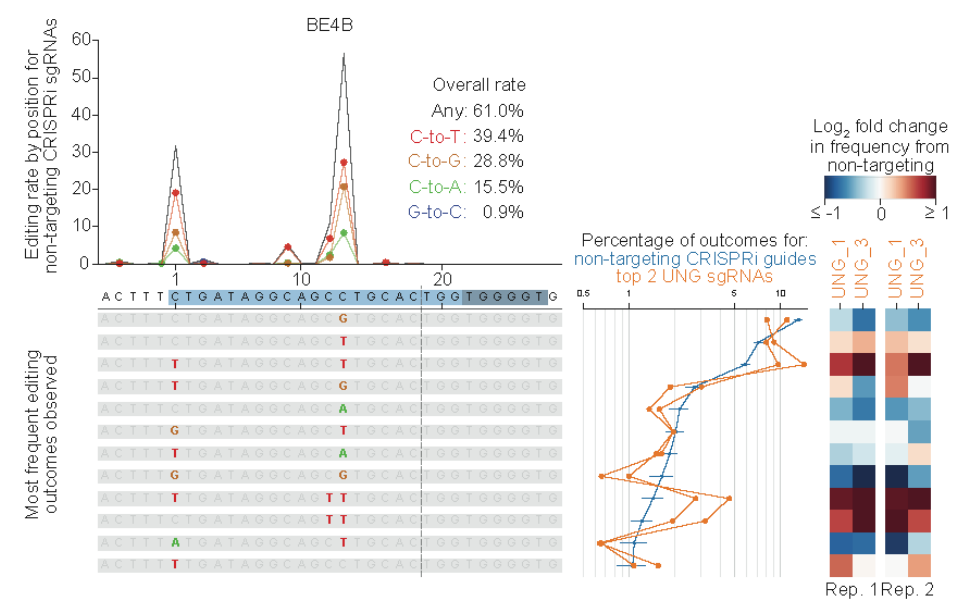

c

perturbed cells

( 1 perturbation per cell)

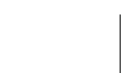

2. Induce base editing by transfecting plasmid-encoding components
(SaCas9 CBEs + sgRNA)

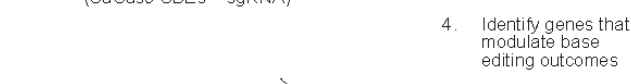

3. Isolate genomic DNA and sequence CRISPRi sgRNAs paired with editing outcomes
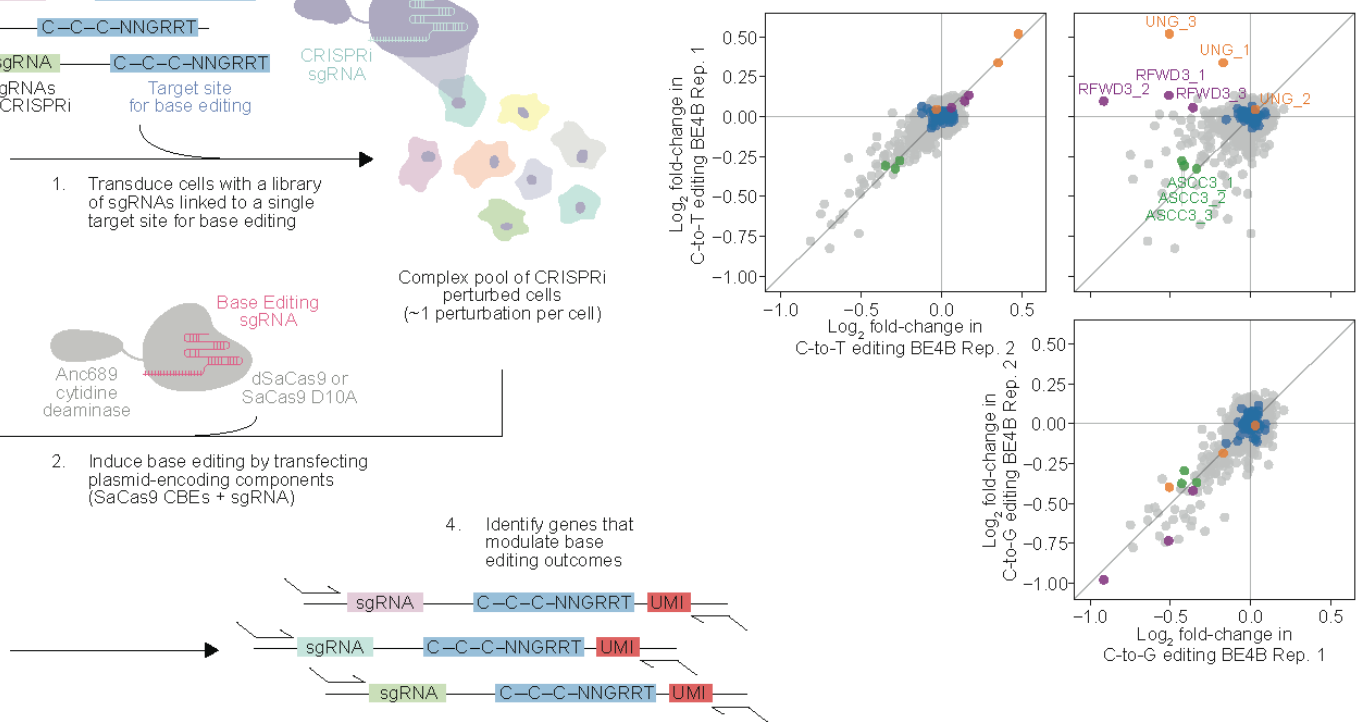

d

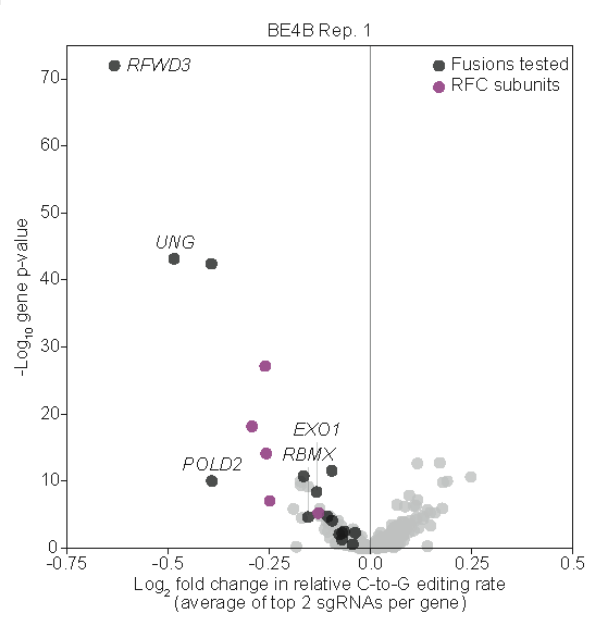

Figure 2. CRISPRi knockdown screen across $\mathbf{4 7 6}$ genes enriched for those with roles in DNA repair identifies candidate regulators of $C \cdot G-t o-G \cdot C$ editing. (a) Schematic of screen design. (b). Summary of base editing outcomes in BE4B (also AC) screen. Bottom left - all editing outcomes containing only point mutations present at $>=1 \%$ frequency for nontargeting CRISPRi guide RNAs. Line plots above the individual outcomes show the total editing frequency (black line) and the frequencies of each single base edit (C-to-T=red, C-to$\mathrm{G}=$ brown, $\mathrm{C}$-to- $\mathrm{A}=$ green, and $\mathrm{G}$-to-C=blue lines) at each position. Line plots to the right show frequencies of outcomes for specific CRISPRi guide RNAs (blue - average of all nontargeting guide +/- standard deviation across individual non-targeting guide RNAs; orange top 2 most active UNG guide RNAs). Heatmaps show $\log _{2}$ fold changes in outcome frequencies for top 2 UNG guide RNAs relative to non-targeting guide RNAs. (c) Log $_{2}$ fold changes in frequency of outcomes containing C-to-T or C-to-G edits for each CRISPRi guide compared to non-targeting guide RNAs. Upper left - comparison of changes in C-to-T editing between two biological replicates. Lower right - comparison of changes in C-to-G editing between replicates. Upper right - comparison of changes in C-to-G editing to changes in C-toT editing in replicate 1 . All guide RNAs with at least 500 recovered UMls in each replicate are plotted. Blue dots: individual non-targeting guide RNAs, orange dots: UNG guide RNAs, 
green dots: ASCC3 guide RNAs, red dots: RFWD3 guide RNAs, grey dots: all other guide RNAs. (d) Effects of gene knockdown on relative C-to-G editing frequencies in BE4B screen. Each dot represents a gene, with the $x$-value representing the average of the two strongest $\log _{2}$ fold changes in normalized C-to-G editing for guide RNAs targeting the gene from the average of all non-targeting guide RNAs, and the $y$-value representing a gene-level $p$-value summarizing the combined statistical significance of all guide RNAs targeting each gene (two-sided, uncorrected for multiple comparisons). Rep.=replicate. 

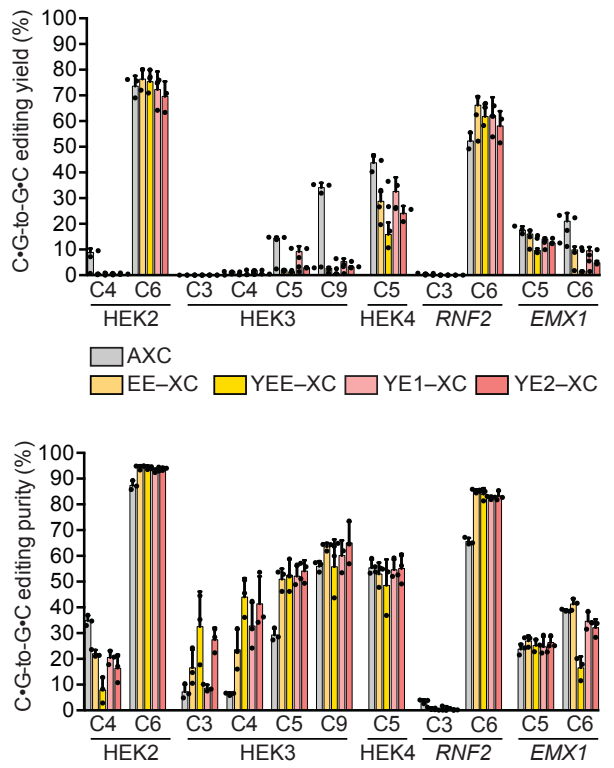

b

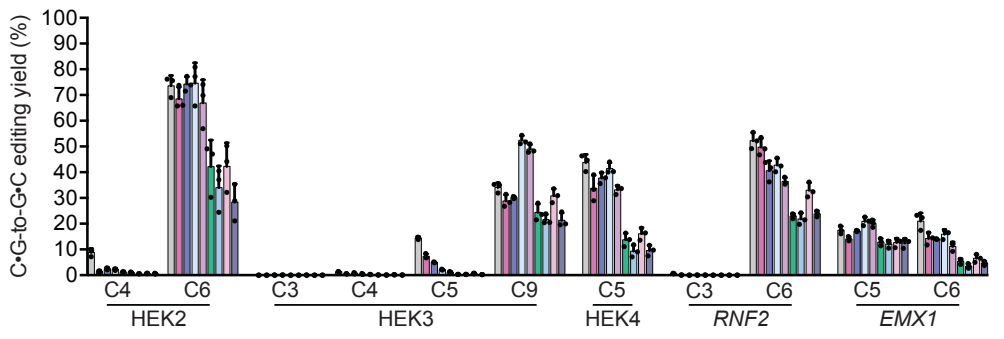

\section{$\square$ AXC}
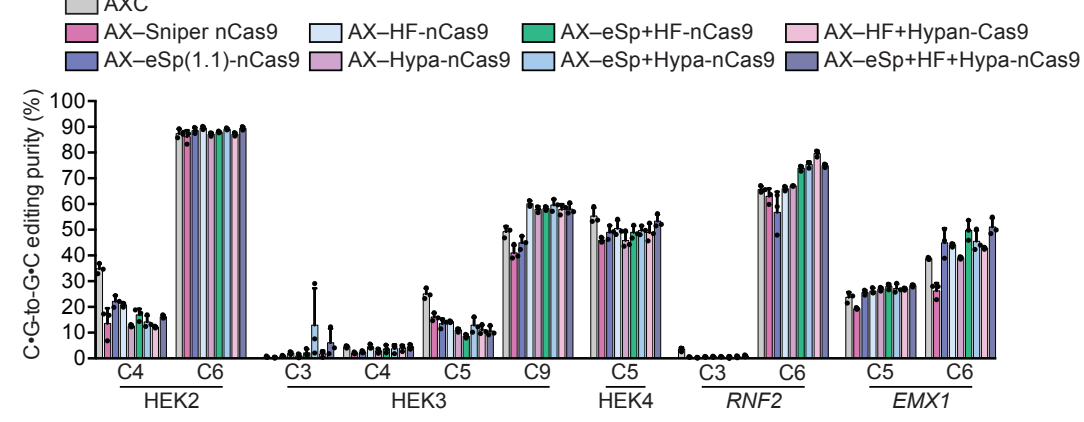

Figure 3. Effect of varying the cytidine deaminase and Cas9 components of CGBEs on C.G-to-G.C editing outcomes in HEK293T cells. (a) C.G-to-G・C editing outcomes for catalytically impaired, narrow-window cytidine deaminases show higher editing purity at HEK2 and RNF2. (b) C.G-to-G・C editing outcomes for high-fidelity Cas9 variants show altered editing windows and improved CGBE performance at some positions. "Cas9" represents the Cas9 D10A nickase variant of each Cas effector. Values and error bars reflect the mean and standard deviation of three biological replicates, shown as individual data points. HEK2=HEK site 2; HEK3=HEK site 3; HEK4=HEK site 4. C4, C6, and similar annotations indicate the in-window target nucleotides where the SpCas9 PAM is at positions 21-23. 

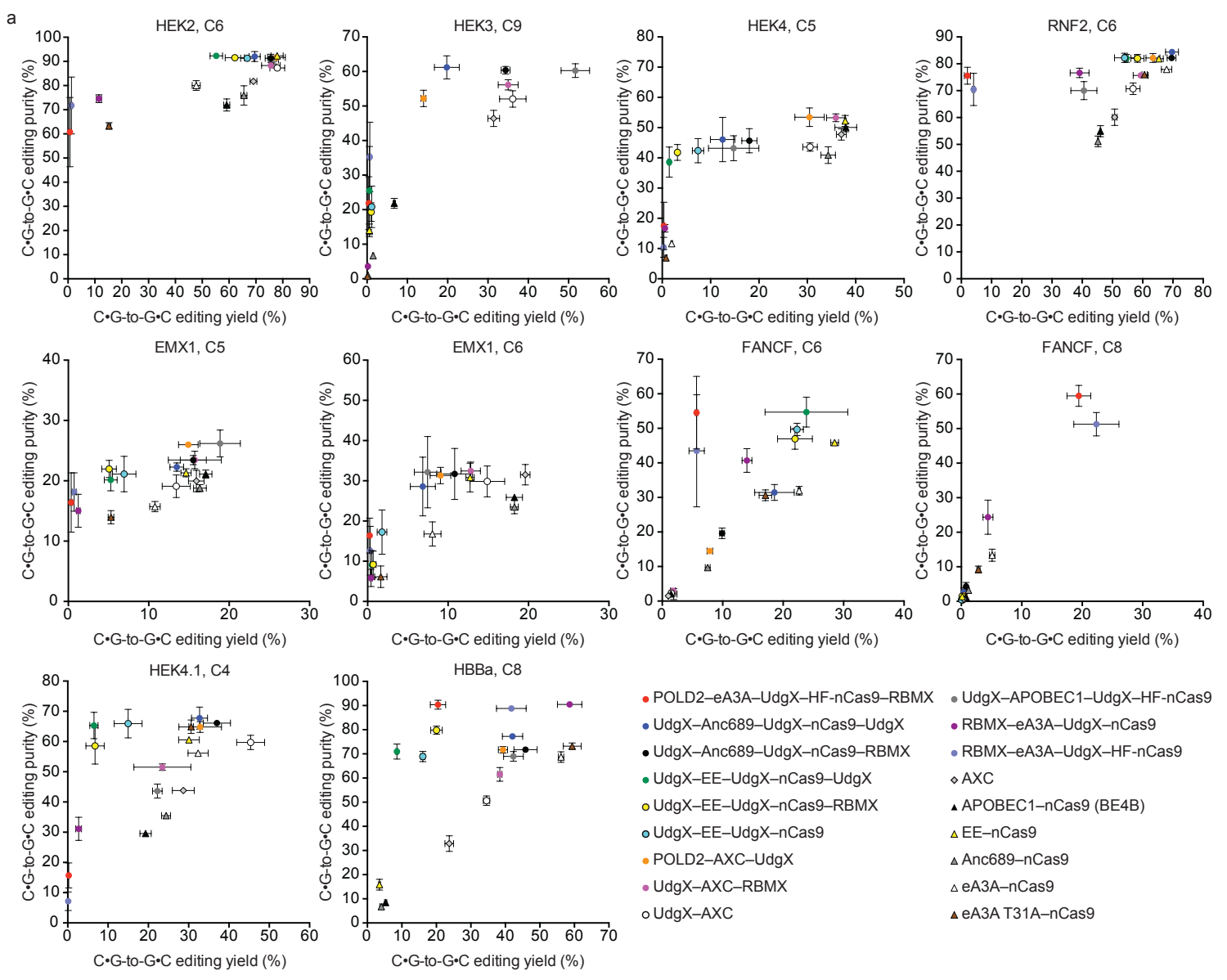
- POLD2-eA3A-UdgX-HF-nCas9-RBMX • UdgX-APOBEC1-UdgX-HF-nCas9
- UdgX-Anc689-UdgX-nCas9-UdgX • RBMX-eA3A-UdgX-nCas9
- UdgX-Anc689-UdgX-nCas9-RBMX • RBMX-eA3A-UdgX-HF-nCas9
- UdgX-EE-UdgX-nCas9-UdgX
$\diamond \mathrm{AXC}$
- UdgX-EE-UdgX-nCas9-RBMX \APOBEC1-nCas9 (BE4B)
- UdgX-EE-UdgX-nCas 9
$\triangle \mathrm{EE}-\mathrm{nCas} 9$
- POLD2-AXC-UdgX $\quad \triangle$ Anc689-nCas9
- UdgX-AXC-RBMX $\quad \triangle$ eA3A-nCas9
○ UdgX-AXC $\quad \triangle$ EA3A T31A-nCas9
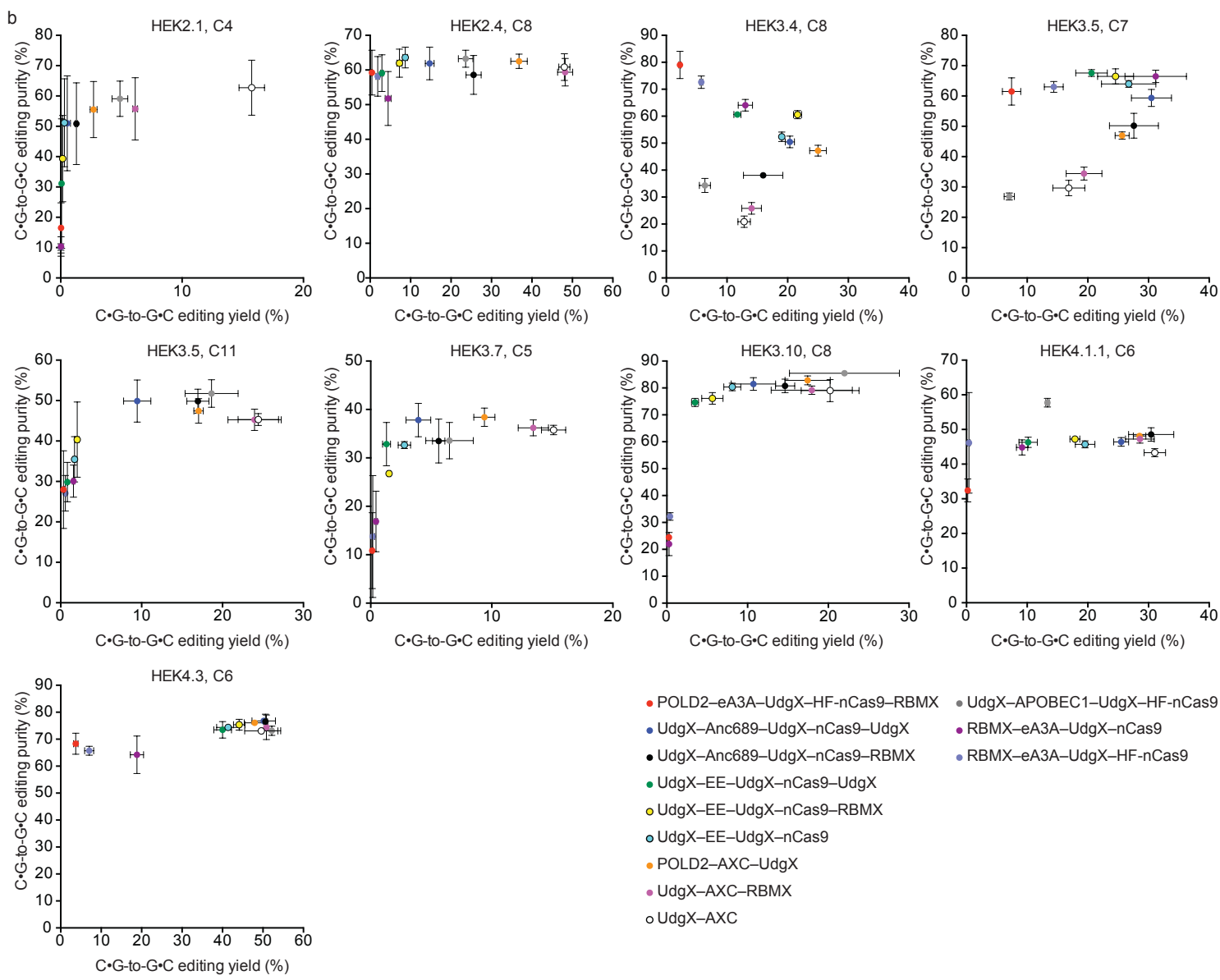

$\begin{array}{ll}\text { - POLD2-eA3A-UdgX-HF-nCas9-RBMX } & \text { • UdgX-APOBEC1-UdgX-HF-nCas9 } \\ \text { - UdgX-Anc689-UdgX-nCas9-UdgX } & \text { • RBMX-eA3A-UdgX-nCas9 } \\ \text { - UdgX-Anc689-UdgX-nCas9-RBMX } & \text { • RBMX-eA3A-UdgX-HF-nCas9 } \\ \text { - UdgX-EE-UdgX-nCas9-UdgX } & \\ \text { - UdgX-EE-UdgX-nCas9-RBMX } & \\ \text { - UdgX-EE-UdgX-nCas9 } \\ \text { - POLD2-AXC-UdgX } \\ \text { - UdgX-AXC-RBMX } \\ \text { - UdgX-AXC }\end{array}$ 
Figure 4. Novel engineered CGBEs with various DNA repair proteins, deaminases, Cas proteins, and architectures offer diverse editing performance on different target sites. (a) C·G-to-G·C editing performance of CGBEs at eight genomic loci in HEK293T cells. (b) Further characterization of $\mathrm{C} \cdot \mathrm{G}$-to-G·C editing outcomes for 12 variants from (a) at various genomic loci in HEK293T cells. Values and error bars reflect the mean and standard deviation of three biological replicates. HEK2=HEK293T cells site 2; HEK3=HEK293T cells site 3; HEK4=HEK293T cells site 4. C nucleotide annotations indicate the target nucleotide positions in the protospacer, where the SpCas9 PAM is at positions 21-23. Editing efficiencies, product purities, and indel frequencies for constructs that were tested but not shown in this figure can be found in Supplementary Data 1. 


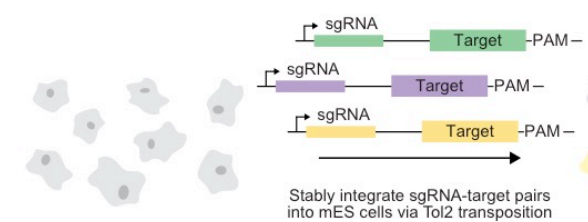

b
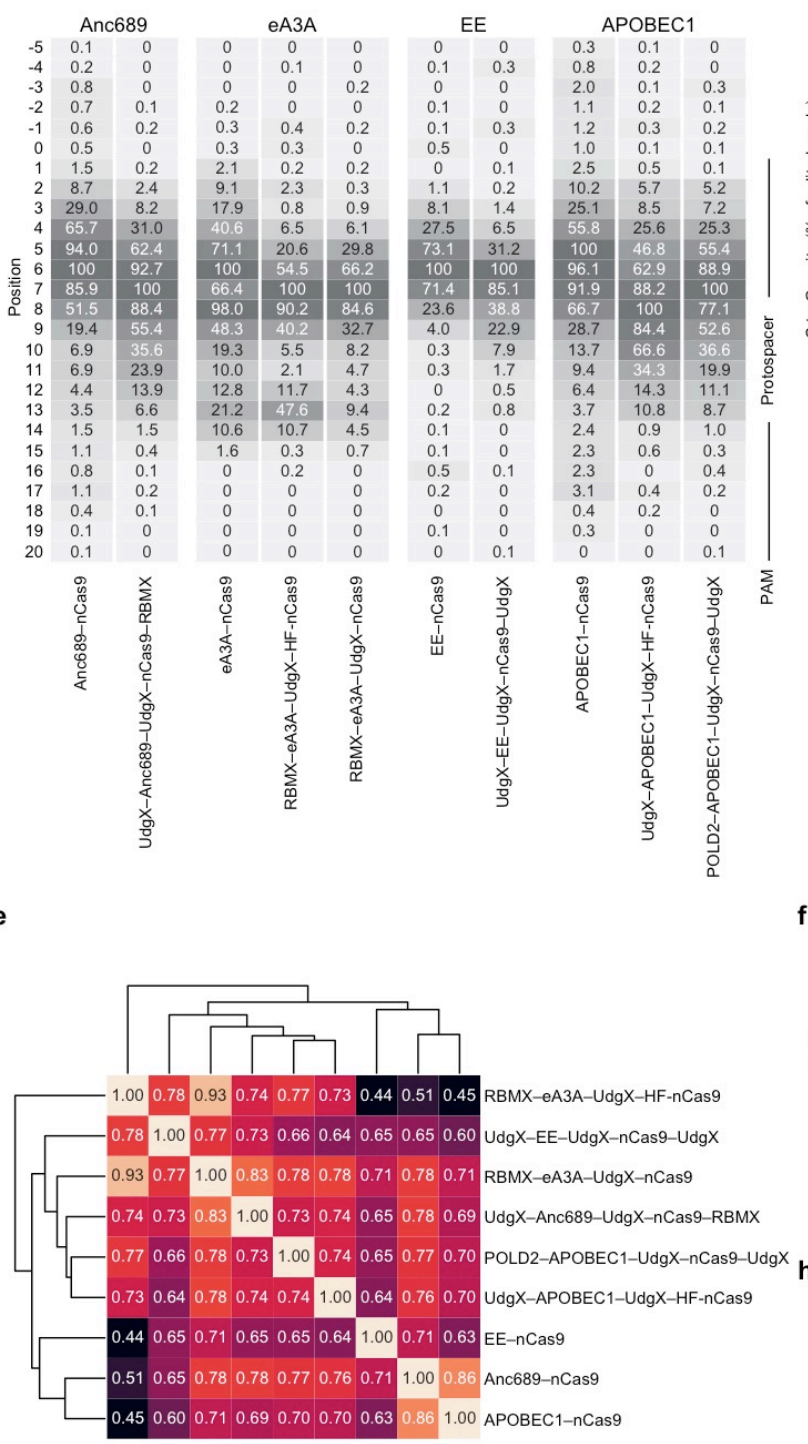

Pearson correlation

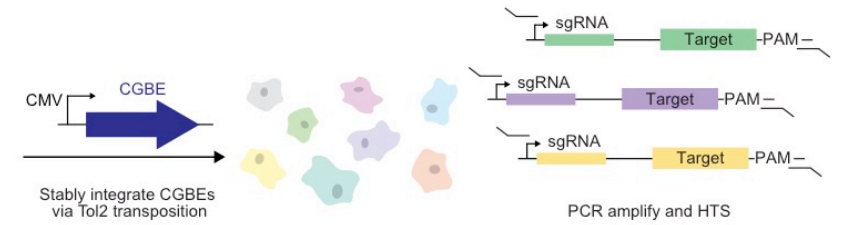

C

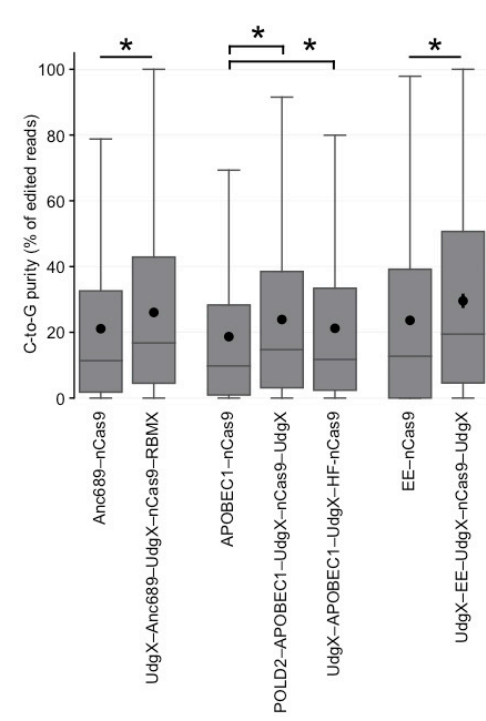

d Protospacer position
56789

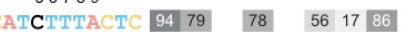
TAATCTACACT \begin{tabular}{llllllll|l}
86 & 77 & 90 & 95 & 86 & 77 & 45 & 82 \\
\hline
\end{tabular} GACTCTTGTCC $72 \quad 63 \quad 65 \quad 64 \quad 96 \quad 50 \quad 55 \quad 62$ $\begin{array}{lllllllll}\text { TGCCCTACCCA } & 36 & 76 & 98 & 68 & 33 & 28 & 62\end{array}$ АСТC CGGGCGA $46 \quad 46 \quad 88 \quad 42 \quad 24 \quad 31 \quad 48 \quad 49$

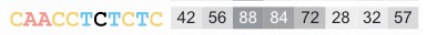
GTCTCTACAGT $26 \quad 55 \quad 88 \quad 58 \quad 73 \quad 23 \quad 46 \quad 60$ $\begin{array}{lllllllll}\text { CAGTCTCCAAC } & 34 & 36 & 84 & 50 & 55 & 13 & 6 & 14\end{array}$ CACTCTCAACA $69 \quad 62 \quad \begin{array}{lllllll}93 & 75 & 53 & 4 & 55\end{array}$ $\begin{array}{lllllllll}\text { GCCTATCTCCA } & 34 & 58 & 46 & 92 & 33 & 37 & 15 & 35\end{array}$ $\begin{array}{llllllll}\text { AGGCCTTTCTG } & 28 & 38 & 98 & 36 & 21 & 37 & 22\end{array}$

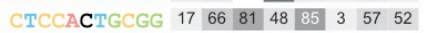
$\begin{array}{llllllllll}\text { GACCAGTCGGA } & 58 & 39 & 23 & 37 & 93 & 36 & 37 & 29\end{array}$ $\begin{array}{lllllllll}\text { TCGACTCTTCA } & 14 & 48 & 82 & 83 & 17 & 26 & 50\end{array}$ ATстCTAGACT $35 \quad 75 \quad 87 \quad 73 \quad 87 \quad 26 \quad 5968$ \begin{tabular}{lllll|lll} 
GACTCTCCTCA & 47 & 52 & 47 & 95 & 16 & 0 & 11
\end{tabular} $\begin{array}{lllllllll}\text { AGGTCTTTCGG } & 79 & 61 & 94 & 67 & 84 & 29 & 46\end{array}$ $\begin{array}{lllllllll}\text { TIACACAAGGG } & 70 & 94 & 90 & 40 & 58 & 95 & 83\end{array}$ \begin{tabular}{ll|l|l|l|l|l|l} 
ACACACCAAGC & 35 & 77 & 50 & 94 & 31 & 77 & 75
\end{tabular} $\begin{array}{llllllllll}\text { ATCACTATTGC } & 29 & 71 & 61 & 92 & 15 & 87 & 70\end{array}$ $\begin{array}{lllllllll}\text { GGCCCCCTCCG } & 63 & 35 & 48 & 28 & 17 & 6 & 88\end{array}$

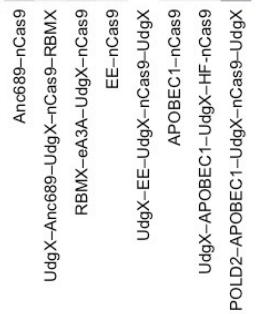

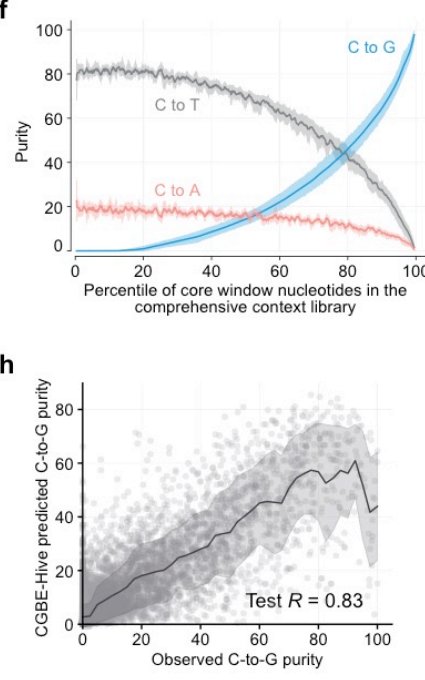

g
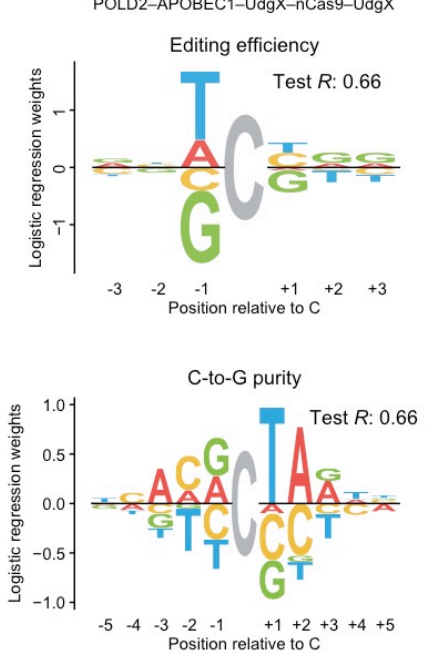

i
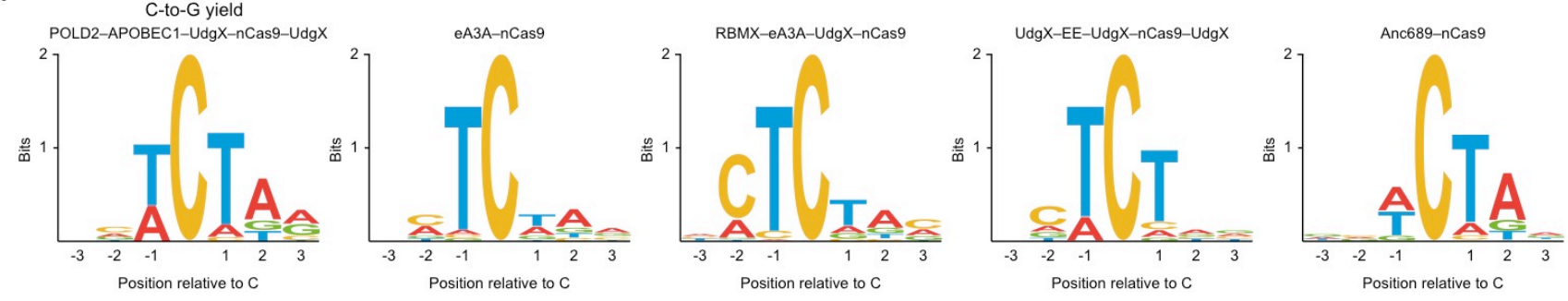
Figure 5. Target library characterization and machine learning modeling of 10 CGBE variants. (a) Overview of genome-integrated target library assay. Libraries of 12,000 or 4,000 pairs of sgRNAs and corresponding target sites are integrated into the genomes of mammalian cells using Tol2 transposase and treated with base editors. Edited cells are enriched by antibiotic selection, and library cassettes are amplified for high-throughput sequencing. (b) Base editing windows. Values are $C \cdot G-$ to-G $\cdot C$ editing efficiencies normalized to a maximum of 100. The protospacer is at positions 1-20, with the SpCas9 PAM at positions 21-23. All data are in mES cells except for eA3A-nCas9, which is in HEK293T cells. (c) $\mathrm{C} \cdot \mathrm{G}-\mathrm{to}-\mathrm{G} \cdot \mathrm{C}$ editing purity in the comprehensive context library in $\mathrm{mES}$ cells. Box plots indicate median and interquartile range, whiskers indicate extrema, and black dots indicate mean. Two-sided Welch's $T$-test ${ }^{*} P \leq 5.1 \times 10^{-9}$. (d) Heatmap of observed C.G-to-G.C purities by CGBE in target contexts from the comprehensive context library in mES cells. Black nucleotides indicate the cytosine for which purity is calculated. Target sites were sorted by outcome variance and manually selected. (e) Clustering of CGBEs based on measured C.Gto-G.C purity in core window cytosines across the comprehensive context library in mESCs. Values are Pearson correlation. (f) Purity of editing outcomes across core window nucleotides in the comprehensive context library, ranked by $C \cdot G-t o-G \cdot C$ purity, averaged across CGBEs in mESCs. Trend lines and shading show the rolling mean and standard deviation across $1 \%$ intervals. (g) Representative sequence motifs for editing efficiency and C.G-to-G.C purity from logistic regression models. The sign of each learned weight indicates a contribution above (positive sign) or below (negative sign) the mean activity. Logo opacity is proportional to the motif's Pearson's $R$ on held-out sequence contexts. (h) Observed C•G-to$\mathrm{G} \cdot \mathrm{C}$ purity across CGBEs in mESCs compared to CGBE-Hive predictions. Trend lines and shading show the rolling mean and standard deviation. (i) Sequence motifs for $C \cdot G-t_{0}-G \cdot C$ editing yield. 


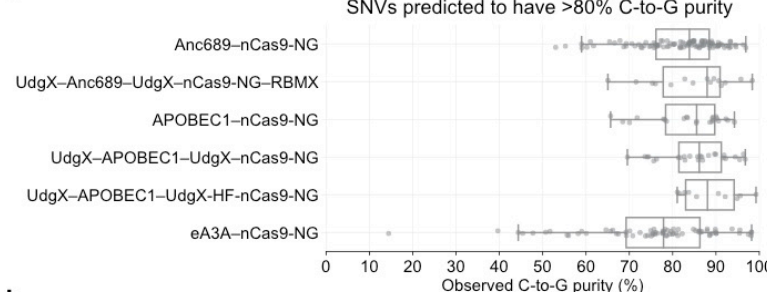

b
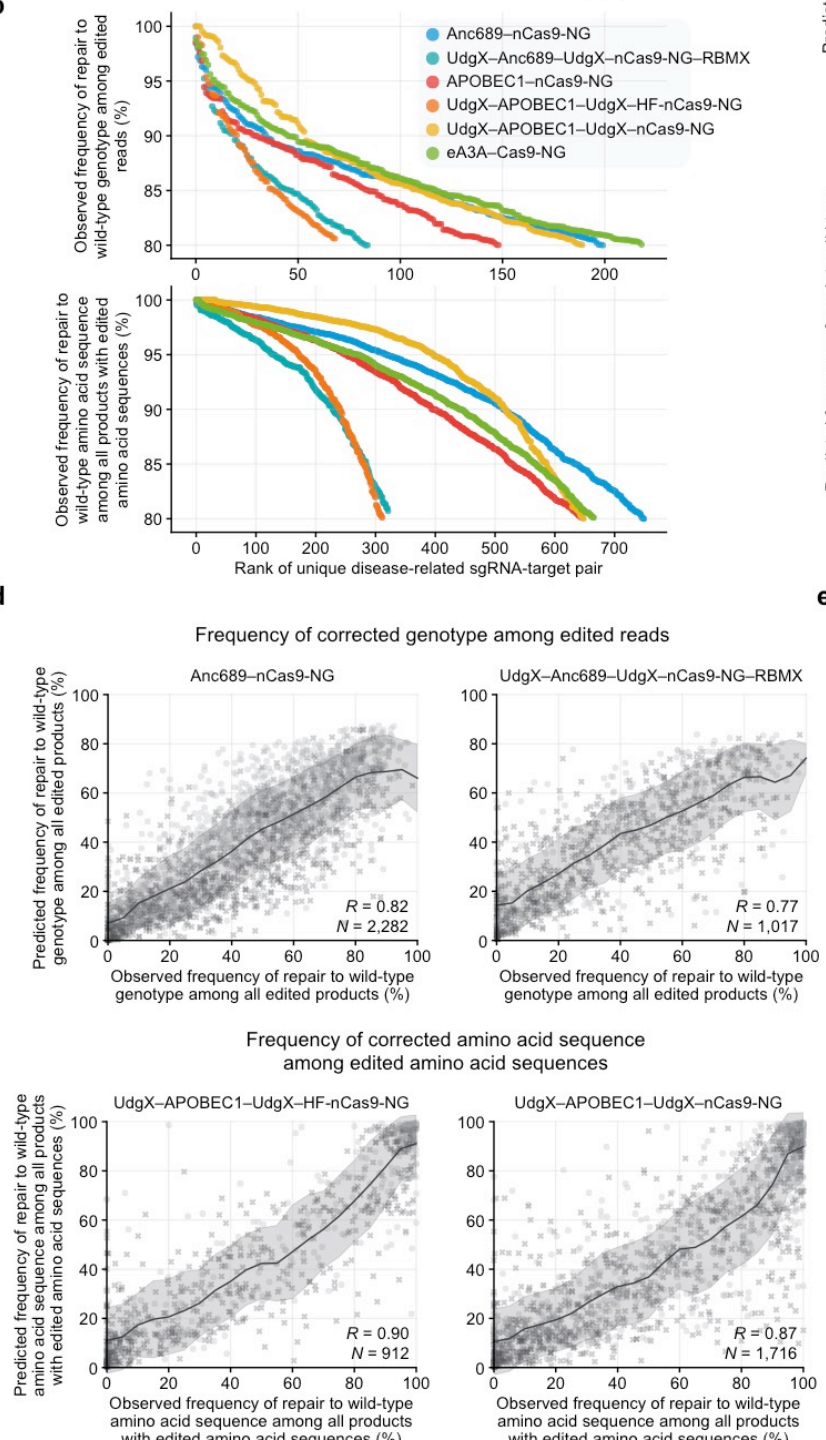
sequences $(\%)$

$\geq 2$ substrate nucleotides in editing window
$-\quad$ No
× Yes

f

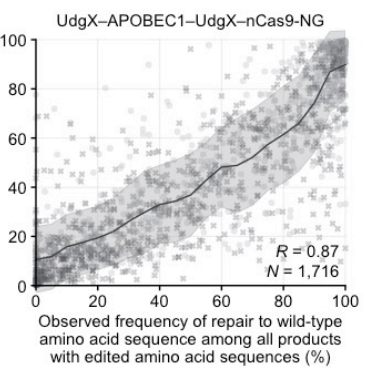

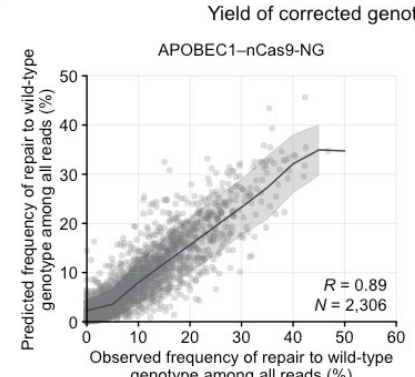

genotype among all reads (\%)
genot
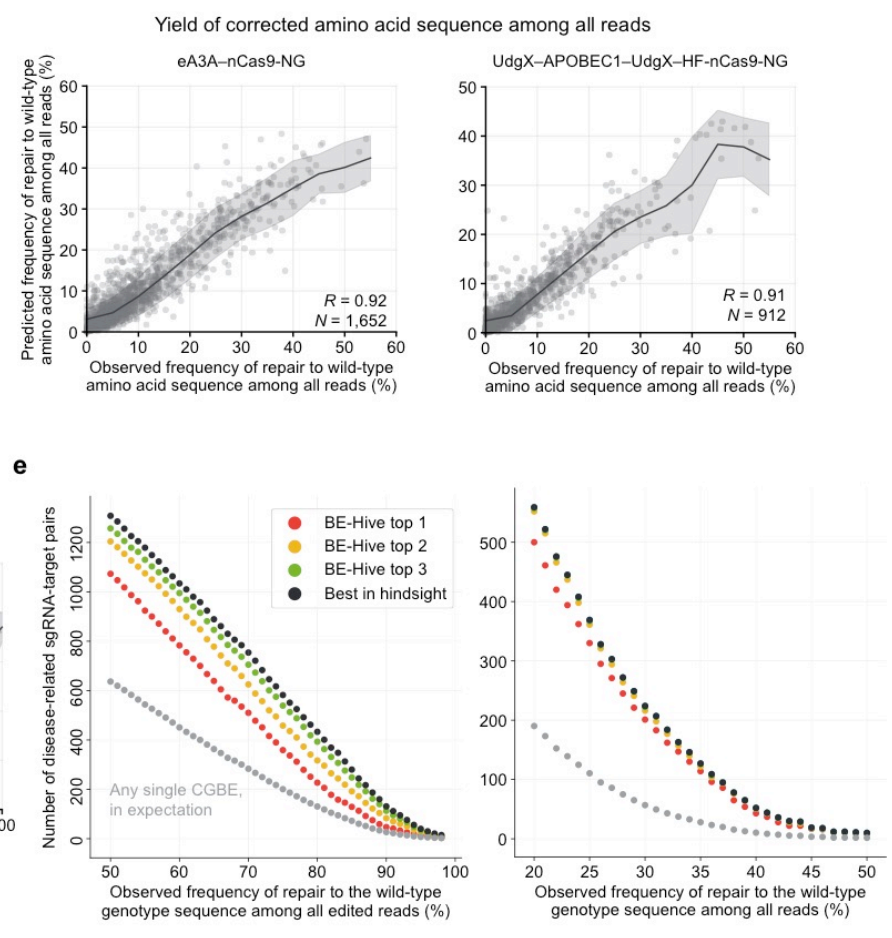

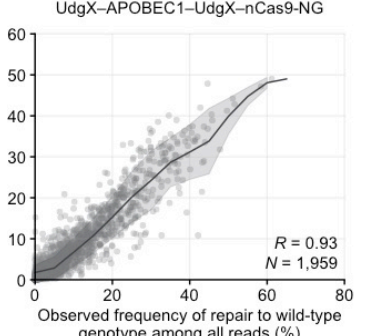

genotype among all reads $(\%)$
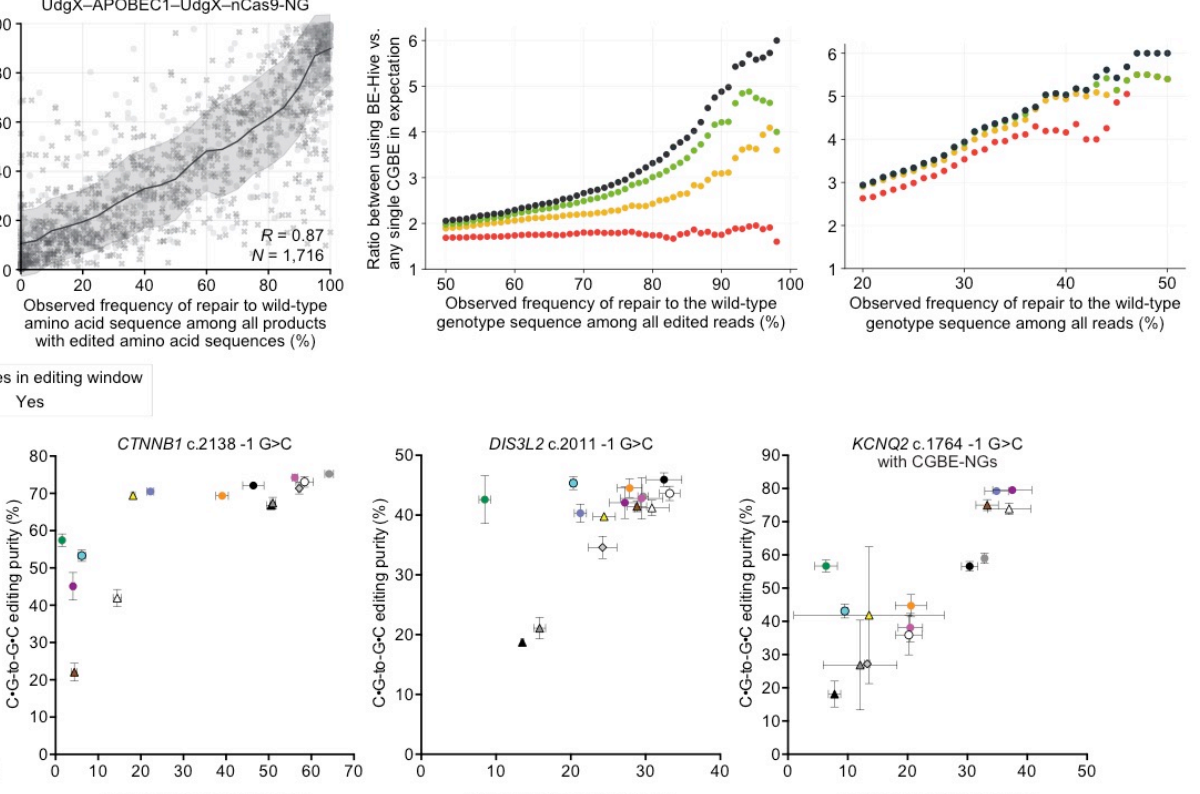

$$
\text { C.G-to-G.C editing yield (\%) }
$$

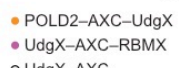

- UdgX-APOBEC1-UdgX-HF-nCas9

$\triangle$ Anc689-nCas9

- UdgX-EE-UdgX-nCas9-UdgX

o UdgX-AXC

- RBMX-eA3A-UdgX-HF-nCas9 $\triangle \mathrm{EE}$-nCas9 
Figure 6. Target library characterization and machine learning modeling of CGBE variants. (a) Observed C-to-G purity by CGBE at SNVs predicted to have $>80 \%$ C-to-G purity. Box plot indicates median and interquartile range, and whiskers indicate extrema. (b) Observed number of disease-related sgRNA-target pairs corrected at varying genotype precision and amino acid precision thresholds by various strategies for selecting CGBEs. See Supplementary Table 3. (c) Comparison of predicted versus observed correction yield of disease-related transversion SNVs in mES cells. Trend lines and shading show the rolling mean and standard deviation. (d) Comparison of predicted versus observed correction precision of disease-related transversion SNVs in mES cells. Trend lines and shading show the rolling mean and standard deviation. (e) Observed number of sgRNA-target pairs containing disease-related transversion SNVs corrected at various thresholds for genotype and amino acid precision. (f) Installation of disease-associated SNPs using CGBEs. 


\section{Methods}

\section{General methods}

DNA oligonucleotides were obtained from Integrated DNA Technologies (except where otherwise specified). All mammalian editor plasmids used in this work were cloned by Gibson assembly according to manufacturer's protocols. Except for the CRISPRi library, plasmids expressing sgRNAs were constructed by ligation of annealed oligonucleotides into BsmBIdigested acceptor vector as previously described ${ }^{24,30}$. Plasmids expressing pegRNAs were constructed by Golden Gate assembly using a custom acceptor plasmid as previously described $^{38}$. Protospacer sequences of sgRNAs used for non-library experiments in this work are listed in Supplementary Table 4. pegRNA protospacer and extension sequences are listed in Supplementary Table 4, tab \#3. Vectors for low-throughput mammalian cell experiments were purified using Plasmid Plus Midiprep kits (Qiagen) or PureYield plasmid miniprep kits (Promega), which include endotoxin removal steps. Cloning of the CBE SaCas9 sgRNA for screening was conducted by KLD assembly according to the manufacturer's protocol using BPK2660 (Addgene \#70709) as a template with the following primers (protospacer is bolded): GGTGTTTCGTCCTTTCCACAAGATA, gCTGATAGGCAGCCTGCACTGGGTTTTAGTACTCTGTAATGAAAATTACAGAATCTAC.

\section{General mammalian cell culture conditions}

HEK293T (ATCC CRL-3216), U2OS (ATTC HTB-96), K562 (CCL-243), and HeLa (CCL-2) cells were cultured and passaged in Dulbecco's Modified Eagle's Medium (DMEM) plus GlutaMAX (ThermoFisher Scientific), DMEM (Gibco), McCoy's 5A Medium (Gibco), RPMI Medium 1640 plus GlutaMAX (Gibco), or Eagle's Minimal Essential Medium (EMEM, ATCC), respectively, each supplemented with $\sim 10 \%(\mathrm{v} / \mathrm{v})$ fetal bovine serum (Gibco, qualified) and 1x Penicillin Streptomycin (Corning). All cell types were incubated, maintained, and cultured at $37{ }^{\circ} \mathrm{C}$ with $5 \% \mathrm{CO}_{2}$. Cell lines were authenticated by their respective suppliers or short tandem repeat profiling and tested negative for mycoplasma. Culturing conditions for library analyses are detailed below. Lentivirus was produced in HEK293T cells by cotransfection with packaging plasmids encoding gag and pol, rev, and tat from HIV-1 and VSVG envelope protein. For these transfections, we used either TransIT®-LT1 Transfection Reagent (Mirus) or Polyethylenimine (PEI; Polysciences, Inc.).

\section{HEK293T tissue culture transfection (non-viral) protocol and genomic DNA preparation}

HEK293T were cells grown, seeded, and transfected as previously described $^{2,3,11,24,30,38}$. Briefly, cells were trypsinized and seeded on 48-well poly-D-lysine coated plates (Corning) to an approximated of $3 \times 10^{5}$ cells per well. $16-24 \mathrm{~h}$ post-seeding, cells were transfected at approximately $60 \%$ confluency with $1 \mu \mathrm{L}$ of Lipofectamine 2000 (Thermo Fisher Scientific) according to the manufacturer's protocols and $750 \mathrm{ng}$ of base editor plasmid and 250 ng of sgRNA plasmid. For Prime editing experiments, non-nicking conditions were carried out with 750 ng of PE2 and 250 ng pegRNA while nicking experiments included an additional $83 \mathrm{ng}$ of nicking sgRNA. $72 \mathrm{~h}$ post-transfection, media was removed, cells were washed with 1x PBS solution (Thermo Fisher Scientific), and genomic DNA was extracted by the addition of $150 \mu \mathrm{L}$ of freshly prepared lysis buffer (10 mM Tris- $\mathrm{HCl}, \mathrm{pH} 7.5 ; 0.05 \%$ SDS; $25 \mu \mathrm{g} / \mathrm{mL}$ Proteinase K (ThermoFisher Scientific)) directly into each well of the tissue culture plate. The genomic DNA•lysis buffer mixture was incubated at $37^{\circ} \mathrm{C}$ for $1 \mathrm{~h}$, followed by an $80^{\circ} \mathrm{C}$ enzyme inactivation step for $30 \mathrm{~min}$. Primers used for mammalian cell genomic DNA amplification are listed in Supplementary Table 4. Protospacer sequences used for each locus are listed in Supplementary Table 4. 


\section{High-throughput DNA sequencing of genomic DNA samples}

Genomic sites of interest were amplified from genomic DNA prepared and sequenced on an Illumina MiSeq as previously described ${ }^{2,3,11,24,30,38}$ with minor modifications. Briefly, amplification primers containing Illumina forward and reverse adapters (Supplementary Table 4) were used for PCR 1, amplifying the genomic region of interest. PCR 1 reactions were performed with $0.5 \mu \mathrm{M}$ of each forward and reverse primer, $1 \mu \mathrm{L}$ of genomic DNA extract, $3 \%$ DMSO, $0.25 \mu \mathrm{L}$ Phusion HS-II polymerase, $5 \mu \mathrm{L}$ Phusion HF buffer, $0.5 \mu \mathrm{L} 10 \mathrm{mM}$ dNTPs, and water to a final volume of $25 \mu \mathrm{L}$. PCR1 reactions were carried out as follows: 98 ${ }^{\circ} \mathrm{C}$ for $2 \mathrm{~min}$, then 32 cycles of $\left[98^{\circ} \mathrm{C}\right.$ for $10 \mathrm{~s}, 61^{\circ} \mathrm{C}$ for $20 \mathrm{~s}$, and $72{ }^{\circ} \mathrm{C}$ for $30 \mathrm{~s}$ ], followed by a final $72{ }^{\circ} \mathrm{C}$ extension for $2 \mathrm{~min}$. Unique lllumina barcoding primer pairs were added to each sample in a secondary PCR reaction (PCR 2). Specifically, $25 \mu \mathrm{L}$ of a given PCR 2 reaction contained $0.5 \mu \mathrm{M}$ of each unique forward and reverse lllumina barcoding primer pair, $1 \mu \mathrm{L}$ of unpurified PCR 1 reaction mixture, $0.25 \mu \mathrm{L}$ Phusion HS-II polymerase, $5 \mu \mathrm{L}$ Phusion HF buffer, $0.5 \mu \mathrm{L} 10 \mathrm{mM}$ dNTPs, and water to a final volume of $25 \mu \mathrm{L}$. The barcoding PCR 2 reactions were carried out as follows: $98^{\circ} \mathrm{C}$ for $2 \mathrm{~min}$, then 12 cycles of $\left[98^{\circ} \mathrm{C}\right.$ for $10 \mathrm{~s}, 61^{\circ} \mathrm{C}$ for $20 \mathrm{~s}$, and $72{ }^{\circ} \mathrm{C}$ for $30 \mathrm{~s}$ ], followed by a final $72{ }^{\circ} \mathrm{C}$ extension for 2 min. PCR products were evaluated by electrophoresis on $2 \%$ agarose gel. PCR 2 products (pooled by common amplicons) were purified by electrophoresis with a $2 \%$ agarose gel using a QIAquick Gel Extraction Kit (Qiagen), eluting with $40 \mu \mathrm{L}$ of water. DNA concentration and library preparation was performed as previously described ${ }^{38}$ by fluorometric quantification (Qubit, ThermoFisher Scientific) and diluted to $4 \mathrm{nM}$ final library concentration before sequencing on an Illumina MiSeq instrument according to the manufacturer's protocols.

Sequencing reads were demultiplexed using MiSeq Reporter (Illumina). Alignment of amplicon sequences to a reference sequence was performed using CRISPResso $2^{39}$ which was run to calculate indels with a window size of 10 . C.G-to-G.C editing purity was calculated as $\mathrm{C} \cdot \mathrm{G}$-to-G・C editing yield $\div[\mathrm{C} \cdot \mathrm{G}$-to-T•A yield $+\mathrm{C} \cdot \mathrm{G}-\mathrm{to}-\mathrm{A} \cdot \mathrm{T}$ yield + indels $]$.

\section{Nucleofection of HAP1, U2OS, K562, and HeLa cells}

Nucleofection was performed on K562, HeLa, and U2OS cells as previously described $^{38}$. 750ng of base editor-expression plasmid and 250ng sgRNA-expression plasmid were nucleofected in a final volume of $20 \mathrm{uL}$ in a 16-well nucleocuvette strip (Lonza). K562 cells were nucleofected using the SF Cell Line 4D-Nucleofector X Kit (Lonza) with $5 \times 10^{5}$ cells per sample (program FF-120), according to the manufacturer's protocol. U2OS cells were nucleofected using the SE Cell Line 4D-Nucleofector X Kit (Lonza) with 3$4 \times 10^{5}$ cells per sample (program DN-100), according to the manufacturer's protocol. HeLa cells were nucleofected using the SE Cell Line 4D-Nucleofector X Kit (Lonza) with $2 \times 10^{5}$ cells per sample (program $\mathrm{CN}-114$ ), according to the manufacturer's protocol. Nucleofiection of HAP1 cells was performed using the same amounts of DNA and final volume in a 16-well nucelocuvette strip; however, HAP1 cells were nucleofected using the SE Cell Line 4D-Nucleofector X Kit (Lonza) with $4 \times 10^{5}$ cells per sample (program DZ-113), according to the manufacturer's protocol. Cells were harvested 72 hours after nucleofection for genomic DNA extraction.

\section{Selection of ten CGBEs for target library characterization}

We sought to select the most representative and diverse subset of CGBEs from endogenous base editing data for 72 CGBEs at eight or 16 endogenous target loci. Briefly, we used a convex relaxation of a quadratic program to find a subset of CGBEs with maximally diverse transversion editing purities and yields. Clustering analysis was used to suggest the number of unique CGBE families. Analytic results were curated manually. The six fusion CGBEs assayed were: PoID2-APOBEC1-UDGX-Cas9-UDGX, RBMX-eA3A- 
UDGX-Cas9, RBMX-eA3A-UDGX-HF-nCas9, UDGX-Anc689-UDGX-Cas9-RBMX, UDGX-APOBEC1-UDGX-HF-nCas9, and UDGX-EE-UDGX-Cas9-UDGX. The four simple CGBE editors were deaminase-nCas9 with eA3A, Anc689, APOBEC1, and EE deaminases. We also assayed eA3A-T31A-nCas9 and eA3A-BEN3-DN13-UGI. eA3A-nCas9, eA3AT31A-nCas9 and eA3A-BEN3- $\triangle N 13-U G I$ were characterized in the comprehensive context library only in HEK293T, while all other CGBEs were characterized in the comprehensive context library only in mESCs. eA3A-nCas9-NG and eA3A-T31A-nCas9-NG were further characterized in the transversion-enriched SNV library in mESCs.

To identify CGBEs with distinct activities, we used quadratic programming to identify a subset of CGBEs with maximum pairwise distances between vectors of $C \cdot G$-to-G.C editing purity and yield across eight or 16 endogenous loci. We also performed hierarchical clustering, and observed that across these endogenous loci, CGBE editing activity primarily clustered by deaminase, though there were also substantial intra-cluster differences in editing activities due to variety in protein fusion architectures that were occasionally larger than intercluster differences, which indicates that CGBE editing activity is affected by both deaminase and protein fusion architectures. As our quadratic programming and clustering methods only consider numerical distances and do not propose subsets optimized for high purity or yield, we manually curated the quadratic programming results by replacing CGBEs with similar neighbors from hierarchical clustering when the neighbors had meaningfully higher purity or yield. Since deaminases, protein fusions, and high-fidelity Cas9 variants are known to alter base editing activity ${ }^{12-15,26}$, we also manually curated our final subset to ensure a diversity of these elements.

\section{CRISPRi library construction}

For our CRISPRi screen we used a platform called Repair-seq, which was developed by Hussmann et al. 2021 using a CRISPRi guide library described elsewhere ${ }^{21}$. This library contains 1513 gene-targeting sgRNAs selected from hCRISPRi-v2. $1^{40}$ and 60 non-targeting controls selected from hCRISPRi-v $2^{40}$ (Supplementary Table 1). Gene-targeted sgRNAs were against 476 genes enriched for ones involved in DNA metabolic processes (e.g., replication, repair, recombination). A minority of the spacer sequences for the gene-targeting sgRNAs in this library were repeated in hCRISPRi-v2.1 and are therefore annotated in

Supplementary Table 1 as targeting multiple gene promoters, with multiple guide identifiers. Our 476 gene count considers only the first set of annotations. Oligonucleotides containing sgRNA targeting sequences were synthesized by Twist Bioscience (Supplementary Table 1).

\section{CRISPRi library cloning}

The guide library was cloned in PAX198 as described elsewhere ${ }^{21}$. This vector was derived from pU6-sgRNA EF1Alpha-puro-T2A-BFP ${ }^{41}$ (Addgene, 60955) through multi-step molecular cloning, as described elsewhere ${ }^{21}$. pAX198 contains a CRISPRi guide expression cassette driven by a modified mouse U6 promoter and ending with a termination signal consisting of 6 Ts. pAX198 also contains a 'target region' for genome editing derived from sequence at the human $H B B$ gene, specifically the second and third exons of $H B B$ (no intron) and part of the 3'UTR (ENST00000647020.1). This region is where we directed Anc689nCas9 and Anc689-dCas9 (see CRISPRi screen cell culture section of Methods). Prior to library cloning, a BstXI site was removed from the target region by site-directed mutagenesis. Library cloning was performed with standard protocols (details available at https://weissmanlab.ucsf.edu/CRISPR/Pooled_CRISPR_Library_Cloning.pdf). Briefly, library oligonucelotides were amplified by PCR (primers 5'-TATGAACCACTAAGGCGTCCAC, 5'TCACCAGCAGACTTTACGCAGC), purified using MinElute Reaction Cleanup Kit (Qiagen), 
digested with Blpl and BstXI, isolated by gel purification, and ligated into a similarly digested expression vector (insert to backbone ratio of $1: 1$ for 16 hours at $16^{\circ} \mathrm{C}$ ). Ligation reactions were electroporated into MegaX DH10B T1 ${ }^{\mathrm{R}}$ Electrocomp ${ }^{\mathrm{TM}}$ cells (ThermoFisher). Cells were grown on agar plates and then scraped into liquid for plasmid purification. The final sgRNA library (AX227) was verified by sequencing.

\section{CRISPRi screen cell culture}

The Repair-seq screens reported here were performed in previously described HeLa cells $^{42}$, which stably express a dCas9-BFP-KRAB fusion (from pHR-SFFV-dCas9-BFPKRAB; Addgene \#46911), in two rounds. The first round of screening evaluated Anc689$\mathrm{nCas9}$. The second round evaluated Anc689-dCas9. Both rounds of screening were conducted as follows: Cells were transduced with guide library (AX227, see CRISPRi library cloning section of Methods) by lentiviral infection. The infections were carried out in DMEM supplemented with $\sim 10 \%(\mathrm{v} / \mathrm{v})$ fetal bovine serum, 1x Penicillin Streptomycin, and $8 \mu \mathrm{g} / \mathrm{mL}$ polybrene at an observed infection efficiency of $\sim 5 \%$ for both Anc689-nCas9 and Anc689dCas 9 , as determined by flow cytometry. Approximately 2 days post transduction, cells were selected in $3 \mu \mathrm{g} / \mathrm{mL}$ puromycin and then, 3 days later, transfected with plasmids for base editing. We performed each screen in replicates, each split one day prior to transfection onto $3015 \mathrm{~cm}$ plates, each containing $\sim 1.2 \mathrm{e} 6$ cells. The transfection procedure was as follows: (1) $25 \mathrm{ng}$ plasmid DNA (75\% editor plasmid; $25 \%$ sgRNA plasmid) was mixed with $3.5 \mathrm{~mL}$ of Opti-MEM (Gibco) and $4.6 \mathrm{~mL}$ Helafect Transfection Reagent (per $15 \mathrm{~cm}$ plate of cells). (2) This mixture was then incubated at room temperature for 20 minutes and (3) added to DMEM (Gibco) supplemented with $\sim 10 \%(\mathrm{v} / \mathrm{v})$ fetal bovine serum $(20 \mathrm{~mL}$ per plate). (4) The prepared media was then used to replace non-transfection media on each plate of cells. Approximately 3 days later, cells were collected for sample preparation. For all arms of screening, $\sim 100 \mathrm{e} 6$ cells or more were collected at a viability of $>85 \%$.

\section{CRISPRi screen sample preparation}

Sequencing libraries were prepared from cells collected at the end of the CRISPRi screens as follows: Genomic DNA was extracted from cell pellets ( 200e6 cells for each replicate of Anc689-nCas9, and 125e6 and 098e6 cells for each of two replicates of Anc689dCas9) using the NucleoSpin® Blood XL kit (Macherey-Nagel, up to $100 \mathrm{e} 6$ cells per column). We fragmented the genomic DNA by digestion with Notl-HF (NEB) and then enriched for editcontaining fragments (1447 bp) by size selecting each sample on a large $0.8 \%$ agarose gel $\left(\mathrm{Ow} \mathrm{I}^{\mathrm{TM}}\right.$ A1 Large Gel System, Thermo Fisher Scientific). Gel electrophoresis was conducted at large-scale (i.e., with wells large enough to hold $1.5 \mathrm{~mL}$ volume per well) to maximize recovery of fragments containing both edited sequences and sgRNA expression cassettes ('target' fragments). Gel preparation details are available at https://weissmanlab.ucsf.edu/CRISPR/IlluminaSequencingSamplePrep_old.pdf. DNA was then isolated from excised regions of the gel using NucleoSpin ${ }^{\circledR}$ Gel and PCR Clean up kit (Macherey-Nagel) with columns placed on a vacuum manifold. Of note, large sample volumes were passed through individual columns using syringe barrels to increase capacity.

Next, size-selected target fragments were prepared for sequencing using custom adaptors compatible with next-generation sequencing technologies from Illumina. These adapters, which contained $12 \mathrm{nt}$ unique molecular identifiers (UMIs), were made by annealing individual DNA oligonucleotides (obtained from Integrated DNA Technologies). The oligonucleotide components were oBA676 (5'-

$G^{*} G^{*} C^{*} C * A G A T C G G A A G A G C G T C G T G T A G G G A A A G A G T G T A G A T C T C G G T G G T C G C C G T$ ATCATT, HPLC purified) and oBA677 (5'-

CAAGCAGAAGACGGCATACGAGATNNNNNNNNNNNNGTGACTGGAGTTCAGACGTGTG 
CTCTTCCGATCT, HPLC purified), where * represents a phosphorothioated DNA base. Prior to ligation, DNA samples were digested with HindlII-HF (NEB). This step removed a 4 nt Notl overhang from one end of the target fragments, leaving only one side available for adaptor ligation. DNA was then purified using SPRIselect Reagent (Beckman Coulter) in a 0.8X reaction, quantified using Bioanalyzer High Sensitivity DNA Analysis (Agilent), and $1 \mu \mathrm{g}$ of the product was ligated to adaptors using enzyme and buffer from the KAPA HyperPrep Kit (Roche) as follows: $30 \mu \mathrm{L}$ ligation buffer, $10 \mu \mathrm{L}$ ligase, adapter at 200:1 adaptor:insert ratio, and PCR-grade water to $110 \mu \mathrm{L}$ total volume. These reactions were incubated at $4^{\circ} \mathrm{C}$ overnight on a thermocycler with lid temperature set to $30^{\circ} \mathrm{C}$.

Following ligation, DNA was purified using SPRIselect Reagent (Beckman Coulter) in two reactions ( $0.65 \mathrm{X}$ followed by $0.8 \mathrm{X}$ ) and target fragments were enriched by PCR as follows: $30 \mathrm{ng}$ of template, amplification primers at $0.6 \mu \mathrm{M}$ final concentration (each), $3 \%$ dimethyl sulfoxide, and 1X KAPA HiFi HotStart ReadyMix (50 $\mu \mathrm{L}$ total volume) run at 1 cycle of 3 minutes at $95^{\circ} \mathrm{C} ; 16$ cycles of 15 seconds at $98^{\circ} \mathrm{C}$, followed by 15 seconds at $70^{\circ} \mathrm{C} ; 1$ cycle of 1 minute at $72^{\circ} \mathrm{C} ; 4^{\circ} \mathrm{C}$ hold. We performed enough PCR reactions to use nearly the entirety of each sample obtained from the ligation and subsequent clean-up reactions. Amplification primers used were oBA679 (5'- CAAGCAGAAGACGGCATACGAGAT) and 5'AATGATACGGCGACCACCGAGATCTACAC-[index]TCGTCGGCAGCGTCAGATGTGTATAAGAGACAGTATCCCTTGGAGAACCACCTTGTTGG. Amplified DNA was purified using SPRIselect Reagent (Beckman Coulter) in a $0.8 \mathrm{X}$ reaction, and index samples were mixed for sequencing. Throughout sample preparation procedures, samples were checked for quality and yield using either a NanoDrop Spectrophotometers (Thermo Fisher Scientific), Agilent 2100 Bioanalyzer system, or by running on a Novex ${ }^{\mathrm{TM}}$ TBE Gel. Sample preparation procedures are also described elsewhere ${ }^{21}$.

\section{CRISPRi screen analysis}

Sequencing of CRISPRi screens, alignment and classification of screen sequencing data, statistical tests of gene significance in Fig. 2d, Supplementary Fig. 4, and Supplementary Table 2, and identification of the top two most active guide RNAs for relevant genes in Figure $2 \mathrm{~b}$ and Supplementary Fig.5a and $5 \mathrm{~b}$ were performed as described in Hussmann et al. $2021^{21}$. Intervals in Supplementary Figure 3c are $95 \%$ Clopper-Pearson intervals of outcome fractions, converted to corresponding $\log _{2}$ fold changes. That is, given $\mathrm{k}$ observed UMls for a given CRISPRi guide in a numerator outcome set out of $n$ total UMls in a denominator outcome superset, the bottom interval $\left(v_{\text {bottom }}\right)$ is the smallest value of the true population proportion of numerator to denominator outcomes such that there is $<=2.5 \%$ chance of observing $>=k$ from Binomial $\left(v_{\text {bottom }}, n\right)$, and the top interval $\left(v_{\text {top }}\right)$ is the largest value of the true population proportion of numerator to denominator outcomes such that there is $<=2.5 \%$ chance of observing $<=\mathrm{k}$ from $\operatorname{Binomial}\left(\mathrm{v}_{\mathrm{top}}, \mathrm{n}\right)$.

\section{Target library cloning}

The target libraries used in this manuscript were previously generated in Arbab, Shen et al. $2020^{12}$. All editors described in this paper were cloned between the N-terminal and Cterminal NLS sequences flanking the eA3A-BE4max (Addgene 152997).

\section{Target library cell culture}

mESC lines used have been described previously and were cultured as described previously ${ }^{43}$. For stable Tol 2 transposon-mediated library integration, cells were transfected using Lipofectamine 3000 (Thermo Fisher) following standard protocols with equimolar amounts of Tol2 transposase plasmid (a gift from R. Sherwood) and transposon-containing plasmid. For library applications, $15-\mathrm{cm}$ plates with $2 \times 10^{7}$ initial cells were used. To generate 
library cell lines with stable Tol2-mediated genomic integration, cells were selected with 150 $\mu \mathrm{g} / \mathrm{mL}$ hygromycin starting the day after transfection and continued for $>2$ weeks. For editing experiments, CGBEs were transfected with Tol2 transposase plasmid using Lipofectamine 3000 and selected with $10 \mu \mathrm{g} / \mathrm{mL}$ blasticidin starting the day after transfection for 4 days before harvesting. We maintained an average coverage of $\geq 300 x$ per library cassette throughout.

\section{Target library high-throughput sequencing}

Library preparation was performed as described in Arbab, Shen et al. $2020^{12}$. Genomic DNA was collected from cells 5 days after transfection, after 4 days of antibiotic selection. For library samples, $20 \mu \mathrm{g}$ gDNA was used for each sample and we maintained an average sequencing depth of $\geq 4,000 x$ per target. All PCRs were performed using NEBNext Ultra II Q5 Master Mix. Samples were pooled using Tape Station (Agilent) and quantified using a KAPA Library Quantification Kit (KAPA Biosystems). The pooled samples were sequenced using Illumina NextSeq.

\section{Target library analysis: data processing}

Sequencing reads were assigned to designed library target sites by locality sensitive hashing ${ }^{12,31}$. Target contexts that were intentionally designed to be highly similar to each other were designed barcodes to assist accurate assignment. Sequence alignment was performed using Smith-Waterman with the parameters: match +1 , mismatch -1 , indel start -5 , indel extend 0 . Nucleotides with PHRED score below 30 were assumed to be the reference nucleotide.

For base editing analysis, aligned reads with no indels were retained for analysis and events were defined as the combination of all possible substitutions at all substrate nucleotides in the target site in a read, where a single sequencing read corresponds to an observation of a single event. Substrate nucleotides were defined as $C$ and $G$ for $C B E s$ and $A$ and $C$ for ABEs.

For indel analysis, reads containing indels with at least one indel position occurring between protospacer positions -6 to 26 were retained, where position 1 is the 5 '-most nucleotide of the protospacer, and 0 is used to refer to the position between -1 and 1 . Reads containing indels without at least six nucleotides with at least $90 \%$ match frequency on both sides of each indel were discarded. Events were defined as indels identified by position, length, and inserted nucleotides occurring in a read. Combination indels were either not observed at all or only at exceedingly low frequencies in endogenous data and were therefore excluded from consideration when analyzing library data.

\section{Target library analysis: base editing profiles}

Base editing profiles were calculated using the same approach as Arbab, Shen et al. $2020^{12}$, using a multi-step procedure to maximize sensitivity. Briefly, single-nucleotide mutation frequencies were tabulated at each target position from sequence alignments in treatment and control data. Treatment data was adjusted for 1) background mutations using untreated control data, 2) sequencing errors, 3) batch effects using other treatment data including published data from Arbab, Shen et al. $2020^{12}$, which primarily helped adjust for rare substitution artifacts from library construction. We then identified mutations that occurred consistently for any editor across replicates to build base editing profiles with sufficient sensitivity to detect rare mutations. We defined cytosine base editing activity as $C$ to $A, G$, or T at positions -9 to 20 and $G$ to $A$ or $C$ at positions -9 to 5 . For all analysis in this work that required tabulating reads with base editing activity, we discarded reads that did not have 
base editing activity according to these broad profiles. Window sizes were calculated at $50 \%$ or greater efficiency relative to the position-wise maximum.

\section{Target library analysis: calculating efficiency and purity}

We required a minimum of 100 reads for calculating editing efficiency, and a minimum of 100 edited reads to calculate purity of editing outcomes. Library members not satisfying these criteria were filtered. The resulting efficiency and purity values were reported as data in the manuscript, and used to train machine learning models. Calculated editing efficiencies and purities were not adjusted for batch effects: instead, our efficiency model is designed to account for batch variation in baseline editing efficiencies by taking it in as optional input. Bystander editing patterns were not found to vary substantially by batch (Arbab, Shen et al., 2020).

\section{Target library analysis: clustering}

CGBEs transversion purities at (target site, nucleotide) tuples in the comprehensive context library were tabulated, and pairwise distances between CGBEs were calculated as the variance explained $\left(R^{2}\right)$ between each pair of CGBEs. Clustering was performed using the L1 distance metric between vectors with the UPGMA clustering algorithm (average linkage).

\section{Target library analysis: identifying targets with diverse editing outcomes}

We calculated a "diversity score" for a target site and substrate nucleotide given observed editing activity values (yield or purity) by a panel of base editors. For a vector of observed values denoted $x$, our diversity score was defined as $\max (x)+2^{*} \operatorname{std}(x)$. We included $\max (x)$ in the score function to encourage library members with very high and very low values to be considered diverse.

To explore the possibility that observed diversity of transversion purity could be explained by analyzing low-abundance outlier library members, we investigated the relationship between the diversity of transversion purity and library member abundance in the transversion-enriched SNV library. We computed a diversity score for each library member, where large values indicate that different CGBEs had different transversion editing purity at that target. We also calculated the relative abundance of each library member in the sequencing data. If library members with extremely high diversity scores were associated with low relative abundance (e.g., if they were explainable by low coverage bottlenecking outliers), their relative abundances should be shifted relative to the background distribution. We tested this hypothesis by comparing the distribution of relative abundance for the top 10 to top 50 library members ranked by diversity score to the full distribution of relative abundances. By Welch's $T$-Test, we found no statistical evidence that high-diversity library members had shifted relative abundance $(P>0.40, N=4,000)$. Furthermore, we observed a mildly positive Pearson correlation $\left(R=0.14, P=4 \times 10^{-14}\right)$ between relative abundance and the diversity score, indicating that across the whole library, library members with higher relative abundance tend to have slightly higher diversity of base editing outcomes. Taken together with other analysis in our paper, we conclude that differences in editing purity by different CGBEs at the same target are better explained by their distinct sequence preferences.

\section{Target library analysis: sequence motif models}

For prediction tasks where the target variable is continuous and has range in $(0,1)$, we first applied a logistic transformation to the data, then used linear regression. For continuous data representing fractions, we discarded values equal to 0 or 1 . For classification tasks, the target variables were either 0 or 1 indicating absence or presence of activity, and we used 
logistic regression. Target variables included the efficiency of C•G-to-T•A editing by CBEs and the purity of cytosine transversions by CBEs. Each of these statistics involves calculating a denominator corresponding to the total number of reads at a target sequence, or the total number of edited reads at a target sequence not including indels. Target sequences with fewer than 100 reads in the denominator were discarded to ensure the accuracy of estimated statistics in the training and testing data. Features were obtained by one-hot-encoding nucleotides per position relative to a substrate nucleotide or to the protospacer. When featurizing data relative to a single substrate nucleotide, each substrate nucleotide within a specified range of positions was used. Ranges used included position 6 only (for the comprehensive context library that contained all NNN-NNN-mers surrounding position 6) and positions 4-8, which was used only when exploratory data analysis indicated that the activity of interest did not vary substantially by position. All nucleotides within a 10-bp radius of the target position were one-hot-encoded. Position was not used as a feature. The data were randomly split into training and test sets at an $80: 20$ ratio. We note that sequence motifs described by these regression models consider each position independently and are intended primarily for visualization.

Motifs for yield were calculated from the top 150 cytosines ranked by C-to-G yield. Column sizes are scaled by their information content.

\section{Target library analysis: base editing efficiency models}

We observed that base editing efficiency varies by experimental batch. To combine replicates across batches, we first performed mean centering and logit transformation at up to 10,638 gRNA-target pairs in each experimental condition separately from the 12kChar library which includes all 4-mers surrounding $A$ or $C$ from protospacer positions 1 to 11 . We discarded data at target sites with fewer than 100 total reads, then averaged values at matched target sites across experimental replicates. Values of negative or positive infinity (resulting from logit of 0 or 1 ) were discarded. The data were randomly split into training and test sets at a ratio of 90:10. Each target site had a single output value corresponding to the mean logit fraction of sequenced reads with any base editing activity. Data points comprising a single replicate were assigned weight $=0.5$. Data points comprising multiple replicates were assigned a weight of the median logit variance divided by the logit variance at that data point, or 1 , whichever value was smaller. In this manner, exactly half of the data points comprising multiple replicates were assigned a weight of 1 , and those with higher variance were assigned a lower weight. We obtained features from each target sequence using protospacer positions -9 to 21 . Features included one-hot encoded single nucleotide identities at each position, one-hot encoded dinucleotides at neighboring positions, the melting temperature of the sequence and various subsequences, the total number of each nucleotide in the sequence, and the total number of $\mathrm{G}$ or $\mathrm{C}$ nucleotides in the sequence.

We used gradient-boosted regression trees from the python package scikit-learn and trained them with tuples of $(x, y$, weights) using the training data. We performed hyperparameter optimization as described in Arbab, Shen et al. $2020^{12}$. We performed 5-fold cross-validation by splitting the training set into a training and validation set at a ratio of 8:1 and retained the combination of hyperparameters with the strongest average cross-validation performance as the final model. We trained models in this manner for each combination of cell-type and base editor. Models were evaluated on the test set which was not used during hyperparameter optimization.

\section{Target library analysis: bystander editing models}

Bystander models were designed and trained using the same approach as Arbab, Shen et al. $2020^{12}$. Briefly, we designed and implemented a deep conditional autoregressive 
model that uses an input target sequence surrounding a protospacer and PAM to output a frequency distribution on combinations of base editing outcomes in the python package PyTorch $^{4}$. The model predicts substitutions at cytosines and guanines for CBEs. The model transforms each substrate nucleotide and its local context using a shared encoder into a deep representation, then applies an autoregressive decoder that iteratively generates a distribution over base editing outcomes at each substrate nucleotide while conditioning on all previous generated outcomes. The encoder and decoder are coupled with a learned position-wise bias towards producing an unedited outcome. The model is trained on observed data by minimizing the KL divergence. Importantly, the conditional autoregressive design is sufficiently expressive to learn any possible joint distribution in the output space, thereby representing a powerful and general method for learning the editing tendencies of any base editor from data. We assembled a dataset where each sgRNA-target pair was matched with a table of observed base editing genotypes and their frequencies among reads with edited outcomes. We discarded data points with fewer than 100 edited reads. We discarded edited genotypes occurring at higher than $2.5 \%$ frequency with no edits at any substrate nucleotides (defined as $C$ for CBEs and $A$ for $A B E s$ ) in positions 1-10. Data from multiple experimental replicates were combined by summing read counts for each observed genotype.

\section{Target library analysis: performance evaluation}

We evaluated machine learning model performance using held-out data. For evaluating models at predicting yield, we used the efficiency model to predict a base editing efficiency score using efficiency summary statistics (mean, std) from the training set. We multiplied the predicted base editing efficiency with the predicted frequency of editing patterns from the bystander model.

\section{Target library analysis: indel quantification}

Indels were quantified using the same approach as Arbab, Shen et al. $2020^{12}$. Indels have strong batch effects in our library assay which can be adjusted within each connected component in the graph defined with nodes representing base editors and edges connecting base editors measured in the same experimental batch. We were able to adjust batch effects for eA3A-nCas9 using two-way ANOVA as previously described since it was included in the same connected component as all BEs previously characterized in Arbab, Shen et al., $2020^{12}$. We were not able to adjust batch effects for all other CGBEs as they were in a separate connected component.

CGBEs are expected to generate indels at higher frequency than canonical base editors as a consequence of generating abasic sites more efficiently. Consistent with this expectation, we previously observed lower base editing to indel (BE:indel) ratios at sites with higher transversion base editing activity. However, we were surprised to observe a positive correlation between $\mathrm{BE}$ :indel ratios and high $\mathrm{C} \cdot \mathrm{G}$-to-G.C editing purity among target library editing outcomes. The geometric mean BE:indel ratio for eA3A-nCas9 was 15:1 across all target sequences, lower than canonical CBEs at 40:1 ${ }^{12}$; however, upon close inspection, we recognized that $B E:$ indel ratios were split dependent upon whether the target sequence was edited with high or low purity. Indeed, the geometric mean BE:indel ratio was below this 15:1 ratio for sites with $<40 \% \mathrm{C} \cdot \mathrm{G}$-to-G・C purity (decreases from $17: 1$ to $12: 1$ as editing purity increases from $0 \%$ to $40 \%$ ) while the geometric average BE:indel ratio increased from 12:1 to $29: 1$ as $C \cdot G-t o-G \cdot C$ purity increased from $40 \%$ to $100 \%$. This surprising positive correlation between $\mathrm{BE}$ :indel ratios and $\mathrm{C} \cdot \mathrm{G}$-to-G.C purity was observed for $11 \mathrm{CGBEs}$ across the comprehensive context and transversion-enriched libraries, with $R=0.05$ to $0.20\left(P<2.4 \times 10^{-6}\right)$. 
No CGBE had a statistically significant negative correlation. This observation suggests that while abasic sites are a common precursor of both indel formation and C.G-to-G・C substitutions and that increased abasic site formation should lead to increases in both indels and $C \cdot G-t o-G \cdot C$ substitutions, target sites particularly amenable to highly pure $C \cdot G$-to-G $\cdot C$ editing preferentially resolve abasic sites against indels. Taken together, these observations highlight the possibility of developing CGBEs with both highly pure C.G-to-G・C editing and high $\mathrm{BE}$ :indel ratios.

\section{Target library analysis: evaluating CGBE-Hive optimization of CGBEs for SNVs} We used six CGBEs for this analysis: Anc689-nCas9-NG, APOBEC1-nCas9-NG, and eA3A-nCas9-NG, UdgX-Anc689-UdgX-nCas9-NG-RBMX, UdgX-APOBEC1-UdgXnCas9-NG, and UdgX-APOBEC1-UdgX-HF-nCas9-NG. For each SNV, we used CGBEHive to identify which CGBE had the highest predicted genotype correction precision or amino acid correction precision among CGBEs that had data for that SNV, which was not always all six CGBEs, as some conditions had different SNVs filtered out due to low read counts or poor data quality. Only SNVs with data for at least three CGBEs were considered. The baseline used was the expectation of the statistic with respect to a uniform distribution over the six CGBEs for each SNV.

\section{Obtaining biological materials}

Plasmids encoding CGBEs and CRISPRi screening materials are available through Addgene. 


\section{Supplementary Information}

Efficient C.G-to-G.C base editors developed using CRISPRi screens, target-library analysis, and machine learning

Luke W. Koblan ${ }^{1,2,3, \dagger}$, Mandana Arbab ${ }^{1,2,3, \dagger}$, Max W. Shen ${ }^{1,2,3,4, \dagger}$, Jeffrey A. Hussmann ${ }^{5-7}$, Andrew V. Anzalone ${ }^{1,2,3}$, Jordan L. Doman ${ }^{1,2,3}$, Gregory A. Newby ${ }^{1,2,3}$, Dian Yang ${ }^{5-7}$, Beverly Mok $^{1,2,3}$, Joseph M. Replogle ${ }^{5-7,8}$, Albert Xu $u^{5-7,8}$, Tyler A. Sisley ${ }^{2}$, Jonathan S. Weissman ${ }^{5,7}$, 9,10*, Britt Adamson ${ }^{5-7,11,12 *}$, David R. Liu ${ }^{1,2,3 *}$

Supplementary Figure 1. HAP1 cells lacking UNG, APE1, REV1, or MLH1 show minimal differences in $\mathrm{C} \cdot \mathrm{G}-\mathrm{to}-\mathrm{G} \cdot \mathrm{C}$ editing outcomes.

Supplementary Figure 2. Effects of polymerase or GFP fusions on $C \cdot G-$ to-G.C editing outcomes.

Supplementary Figure 3. Additional CRISPRi screen outcomes.

Supplementary Figure 4. Effects of gene knockdown on editing outcomes by category.

Supplementary Figure 5. Phenotypes for CRISPRi guide RNAs targeting RECQL and HLTF.

Supplementary Figure 6. Fusion of proteins to AXC scaffold alters $C \cdot G-t o-G \cdot C$ editing outcomes in HEK293T cells.

Supplementary Figure 7. Optimization of linkers between CGBE components.

Supplementary Figure 8. Split-intein and unsplit CGBE variants edit with similar yield and product purity.

Supplementary Figure 9. Performance of CGBE variants in K562, U2OS, and HeLa cells.

Supplementary Figure 10. CGBE activity using Cas9-NG ${ }^{1}$.

Supplementary Figure 11. On-target CGBE editing profiles for off-target analyses. $\quad 16$

Supplementary Figure 12. Transversion-enriched SNV library analysis.

Supplementary Figure 13. Comparison of CGBEs developed in this study with recently described CGBEs.

Supplementary Figure 14. Comparison of prime editing and CGBE editing outcomes.

Supplementary Figure 15. Off-target DNA editing activities of CGBEs. 
Supplementary Discussion 1-6

Supplementary Sequences

Supplementary References

Supplementary Tables and Data provided as separate files:

Supplementary Table 1. CRISPRi sgRNA library.

Supplementary Table 2. Changes in base editing outcomes for all genes in CRISPRi screens

Supplementary Table 3. Base editing outcomes in a library of disease-related alleles correctable by editing $C \cdot G$ to $G \cdot C$ or to $A \cdot T$

Supplementary Table 4. CGBE targets, amplicons, and oligos used for this study

Supplementary Data 1. All C $\cdot G$-to-G・C editing yield, purity, and indel outcomes for all experiments in this manuscript. T-tests can be generated for any pairwise comparison in this file. 

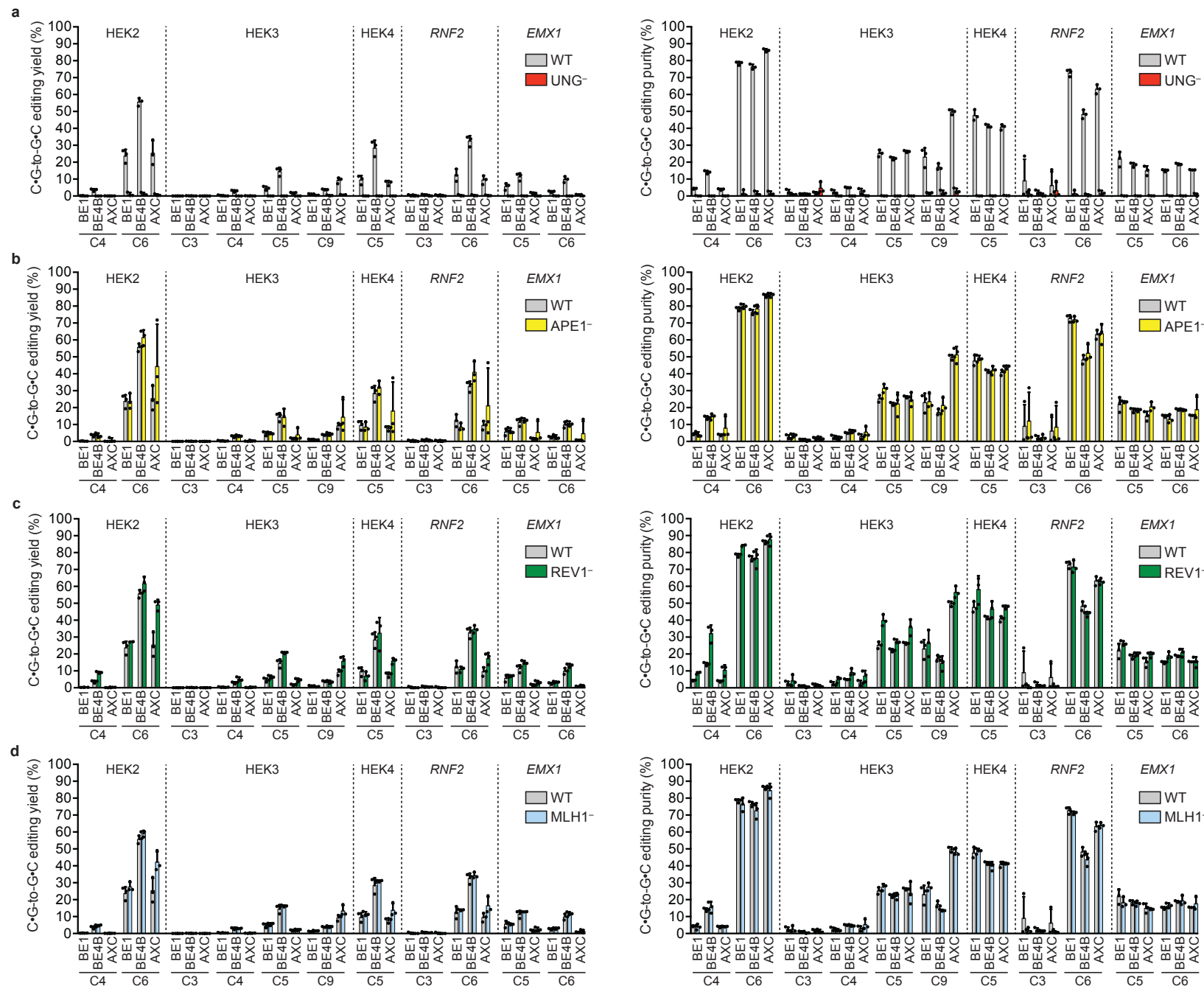

Supplementary Figure 1. HAP1 cells lacking UNG, APE1, REV1, or MLH1 show minimal differences in C.G-to-G.C editing outcomes. $C \cdot G-t o-G \cdot C$ editing yield and product purity of BE1 (nuclease inactive, no UGIs), BE4B (D10A nickase, no UGls; also AC) and AXC (APOBEC1-UdgX-Cas9 D10A, the prototype CGBE), in HAP1 knockout haploid human cell lines lacking (a) UNG, (b) APE1, (c) REV1, and (d) MLH1. Values and error bars reflect the mean and standard deviation of three biological replicates, shown as individual data points, except HEK2 editing in REV1- cells shows two biological replicates. HEK2=HEK293T cells site 2; HEK3=HEK293T cells site 3; HEK4=HEK293T cells site 4. C4, C6, and similar annotations indicate the in-window target nucleotides where the SpCas9 PAM is at positions 21-23. 

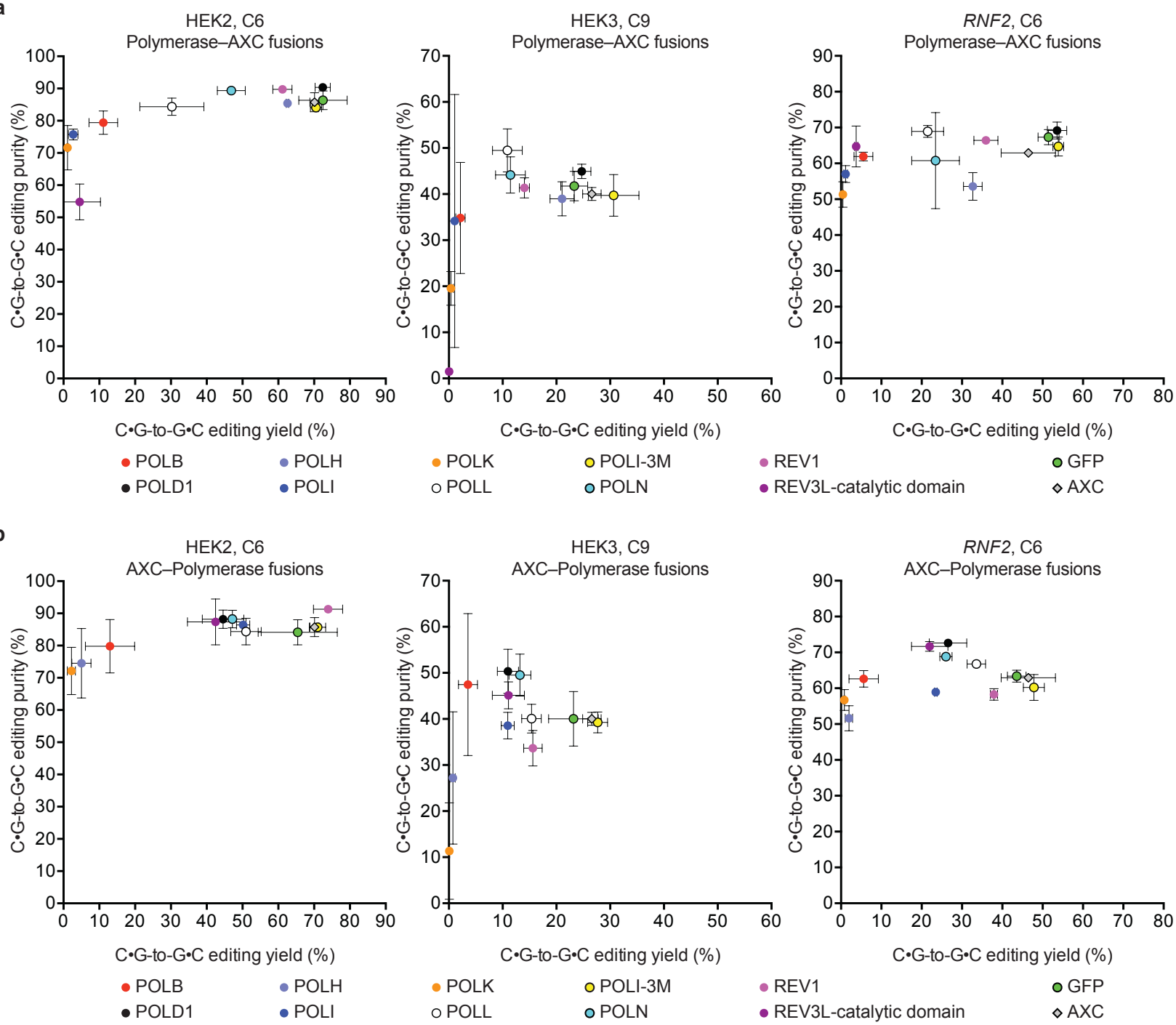

Supplementary Figure 2. Effects of polymerase or GFP fusions on C.G-to-G・C editing outcomes. (a) C.G-to-G·C editing outcomes in HEK293T cells using $\mathrm{N}$-terminal polymerase fusions to AXC (Polymerase-AXC). GFP-AXC and AXC are shown as controls. (b) C.G-to$G \cdot C$ editing outcomes in HEK293T cells using C-terminal polymerase fusions to AXC (AXCPolymerase). AXC-GFP is shown as a control with AXC reproduced from (a) for ease of comparison. $\mathrm{C} \cdot \mathrm{G}$-to-G.C editing yield is shown on the $\mathrm{x}$-axis and product purity is shown on the $y$-axis. Window position annotations indicate the in-window target nucleotides where the SpCas9 PAM is at positions 21-23. Values and error bars reflect the mean and standard deviation of three biological replicates. HEK2=HEK293T cells site 2; HEK3=HEK293T cells site 3; HEK4=HEK293T cells site 4. 
a
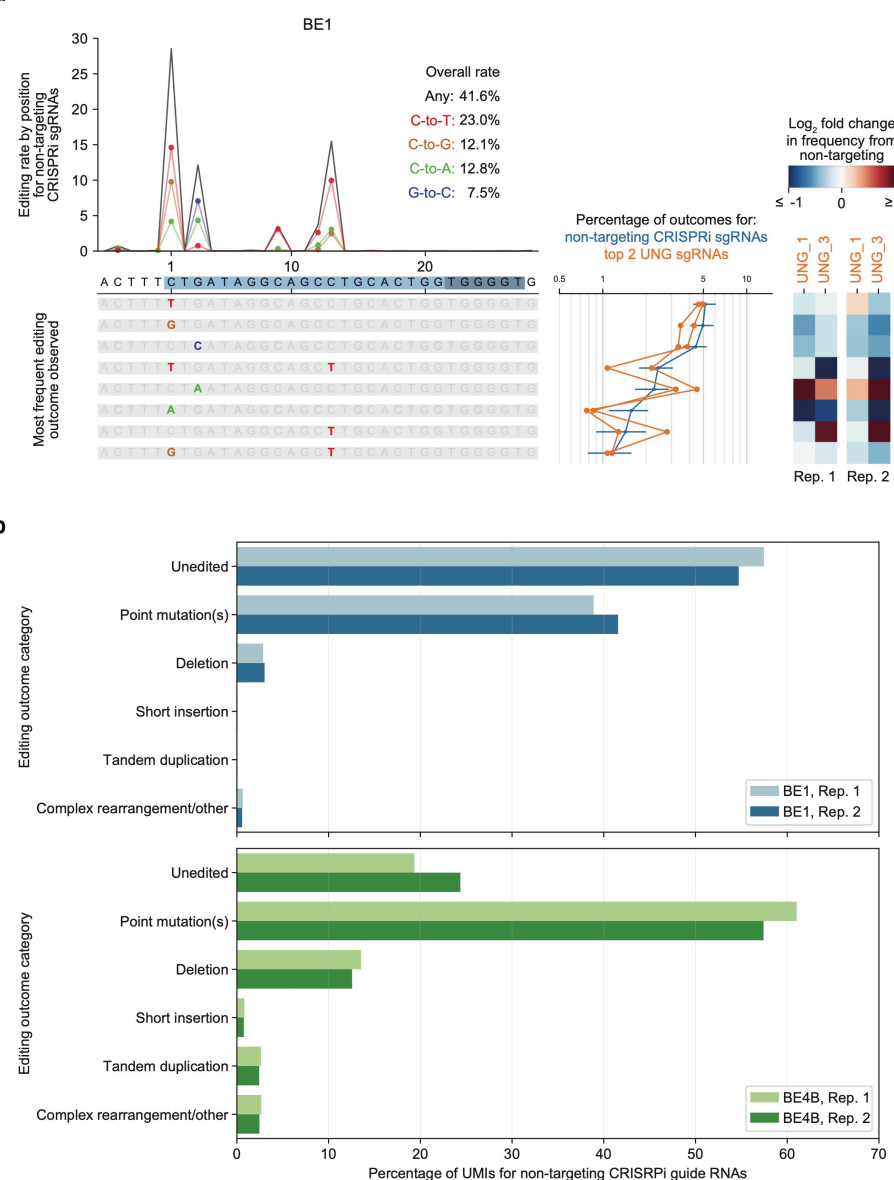
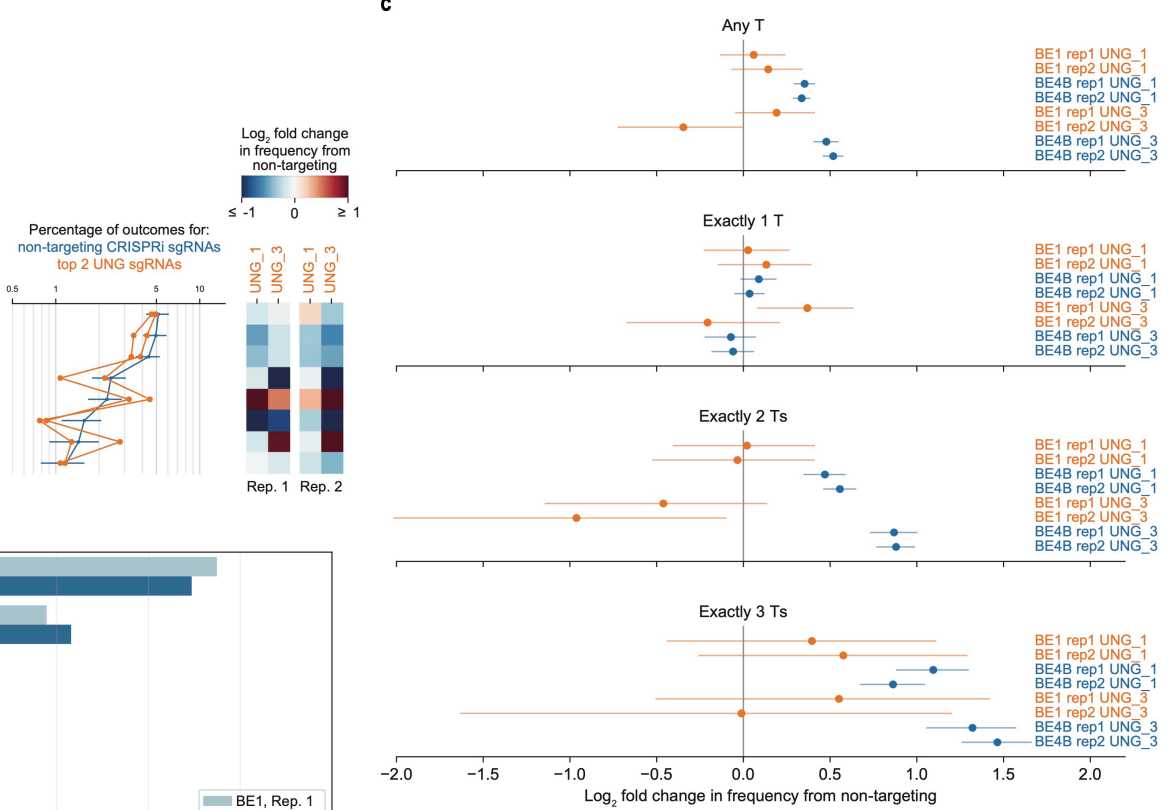

Supplementary Figure 3. Additional CRISPRi screen outcomes. (a) Summary of base editing outcomes in BE1 screen. Bottom left: all editing outcomes containing only point mutations present at $\geq 1 \%$ frequency for non-targeting control CRISPRi guide RNAs, ordered by frequency. Line plots above the individual outcomes show the total editing frequency (black line) and the frequencies of each type of single-base mutation (C-to-T=red, C-to$\mathrm{G}=$ brown, C-to-A=green, and G-to-C=blue) at each position. Right: frequencies of outcomes for specific CRISPRi guide RNAs (blue=mean \pm SD of all non-targeting CRISPRi guide RNAs; orange=the top two most active UNG-targeting CRISPRi guide RNAs). Heatmaps show $\log _{2}$ fold changes in outcome frequencies for the two most active UNG-targeting CRISPRi guide RNAs relative to non-targeting control CRISPRi guide RNAs. (b) Frequency of editing outcome categories in screens. (c) $\log _{2}$ fold changes in frequency of specific editing outcomes containing C-to-T mutations for UNG-targeting CRISPRi guide RNAs in BE1 (orange) and BE4B (blue) screens. Intervals are 95\% Clopper-Pearson binomial confidence intervals for the observed frequencies of each outcome category given the number of UMls recovered for each CRISPRi guide RNA, converted into $\log _{2}$ fold changes. Rep.=replicate. 

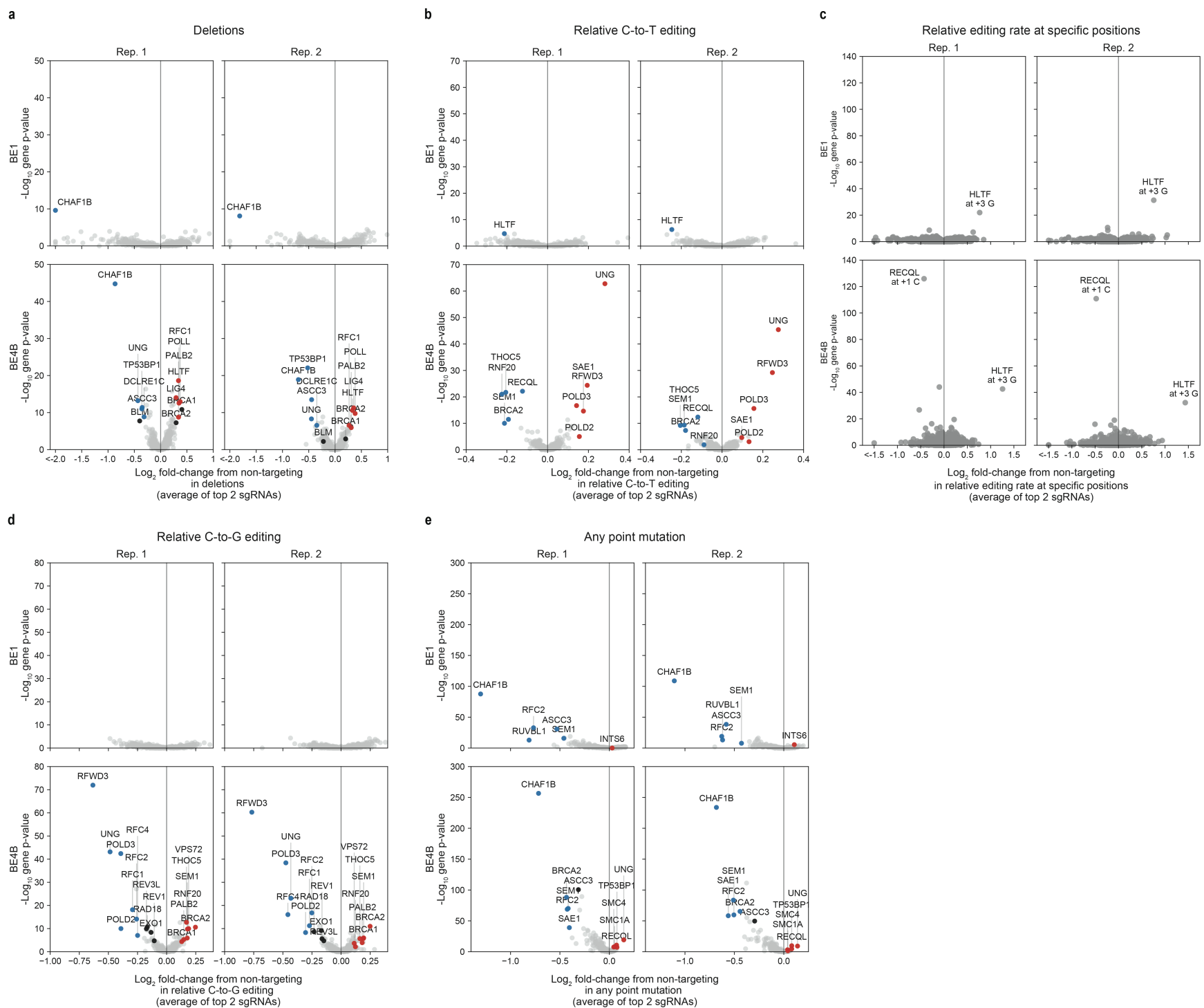

Supplementary Figure 4. Effects of gene knockdown on editing outcomes by category. Each dot in scatter plots represents a gene, with the $x$-value representing the average of the two strongest $\log _{2}$ fold changes in the frequency of the relevant outcome category for CRISPRi guide RNAs targeting that gene compared to the average of all non-targeting guide RNAs, and the $y$-value representing a gene-level $p$-value summarizing the combined statistical significance of all guide RNAs targeting each gene. In each panel, the genes with the largest negative (blue) and positive (red) average $\log _{2}$ fold changes across two replicates that achieve a p-value less than or equal to $10^{-5}$ in either replicate are labeled (up to 5 genes labeled). Additional genes with phenotypes referenced in the text are also labeled (black). Pvalues represent two-sided tests without correction for multiple comparisons. Outcome categories are as follows: (a) Outcomes containing any deletion. (b) Outcomes containing C.G-to-T•A point mutations, as a fraction of outcomes containing any point mutations. (c) Outcomes containing point mutations at specific positions, as a fraction of outcomes containing any point mutation (where the SaCas9 NNGRRT PAM occupies positions 22-27). The 5 most highly modified positions were included. (d) Outcomes containing C.G-to-G・C point mutations, as a fraction of outcomes containing any point mutations. (e) Outcomes containing only point mutations. Rep.=replicate. 


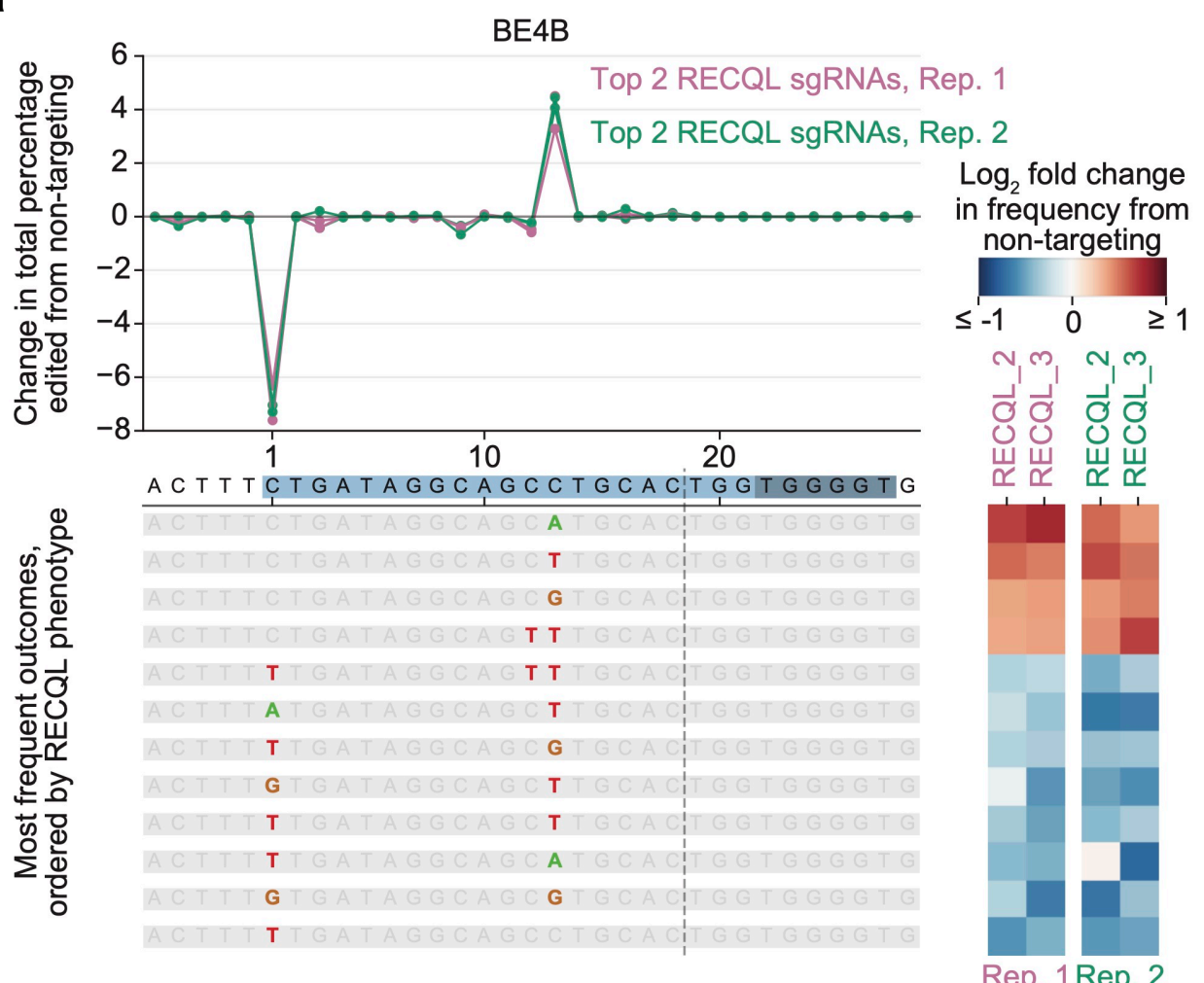

b
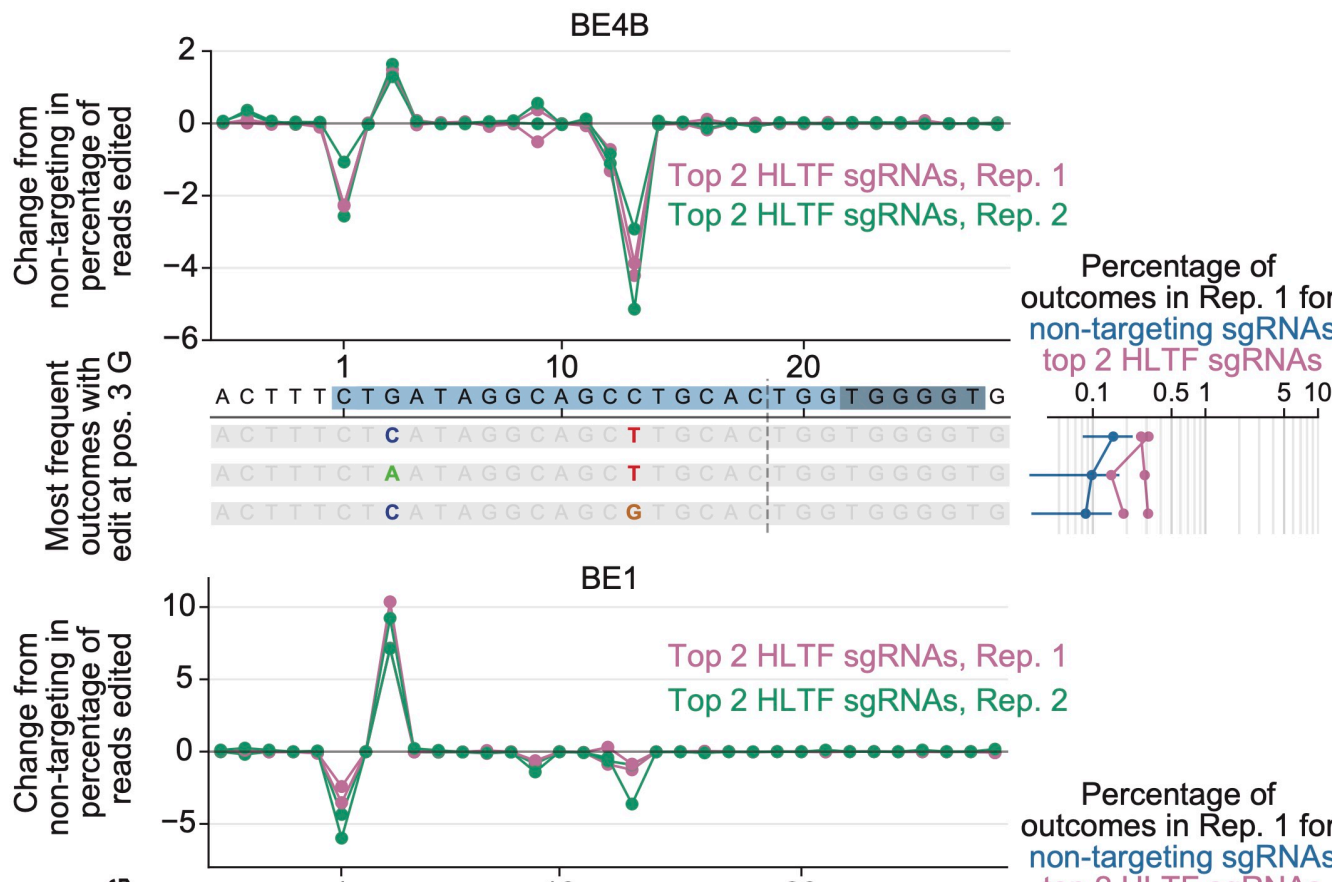

Percentage of outcomes in Rep. 1 for: non-targeting sgRNAs

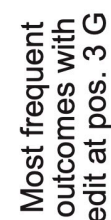

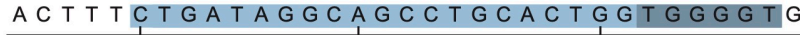

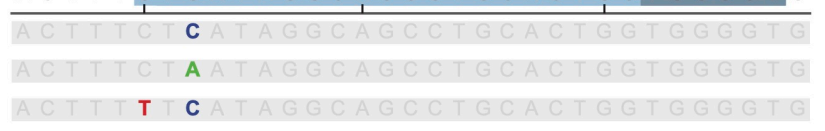
top 2 HLTF sgRNAs

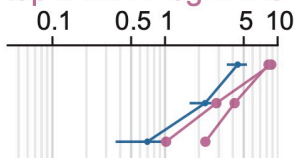

$\log _{2}$ fold change in frequency from non-targeting

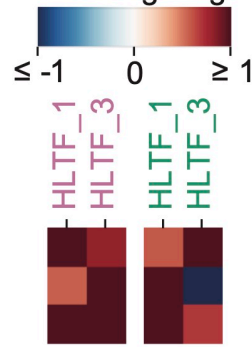

Rep. 1 Rep. 2

$\log _{2}$ fold change in frequency from non-targeting

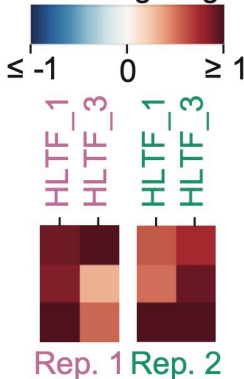

Supplementary Figure 5. Phenotypes for CRISPRi guide RNAs targeting RECQL and HLTF. (a) Effect of RECQL knockdown on editing window in BE4B screens. Bottom left: most frequent point mutation editing outcomes, ordered by average $\log _{2}$ fold changes in frequency 
from non-targeting caused by two most active RECQL guide RNAs in replicate 1. Heatmaps show $\log _{2}$ fold changes from non-targeting guide RNAs. Line plots above outcome diagrams show differences in total editing rates at each position between the top two CRISPRi RECQL guide RNAs and non-targeting guide RNAs. (b) Effect of HLTF knockdown on editing window in BE4 (top) and BE1 (bottom) screens. Diagrams show the three most frequent outcomes with an edit at position +3 (where positions 22-27 are the SaCas9 NNGRRT PAM) for nontargeting CRISPRi guide RNAs. Line plots above outcomes show differences in total editing rates at each position between HLTF guide RNAs and non-targeting guide RNAs. Line plots to the right of outcomes show frequencies of outcomes for specific CRISPRi guide RNAs in replicate 1 (blue=average frequency of each outcome across all non-targeting guide RNAs +/- standard deviation across individual non-targeting guide RNAs; pink=frequency of each outcome for top 2 HLTF guide RNAs). Heatmaps show $\log _{2}$ fold changes from non-targeting CRISPRi guide RNAs. Rep.=replicate. 

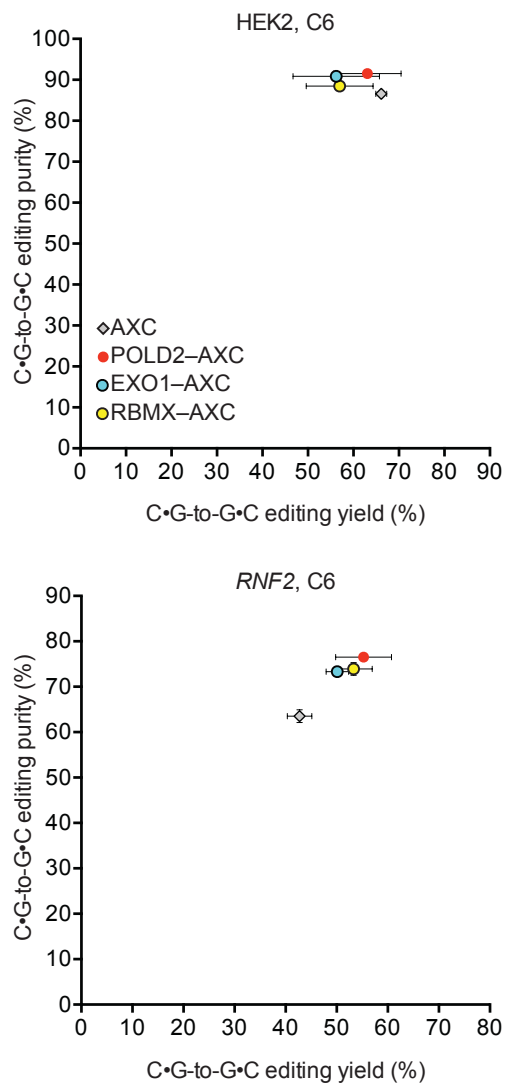

b

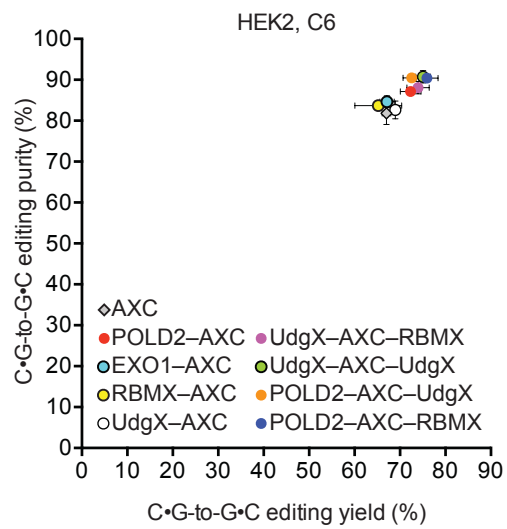

$\mathrm{C} \cdot \mathrm{G}-\mathrm{to}-\mathrm{G} \cdot \mathrm{C}$ editing yield (\%)

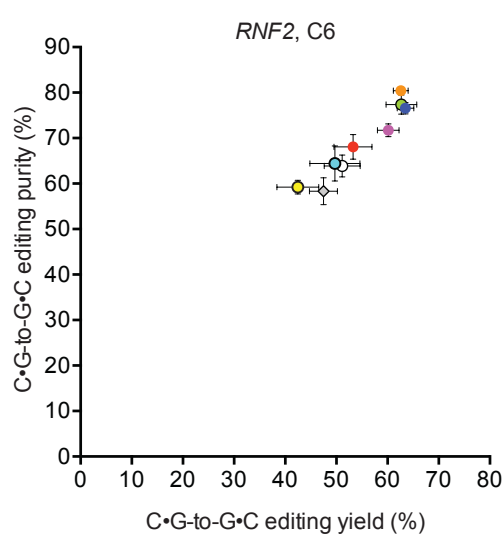

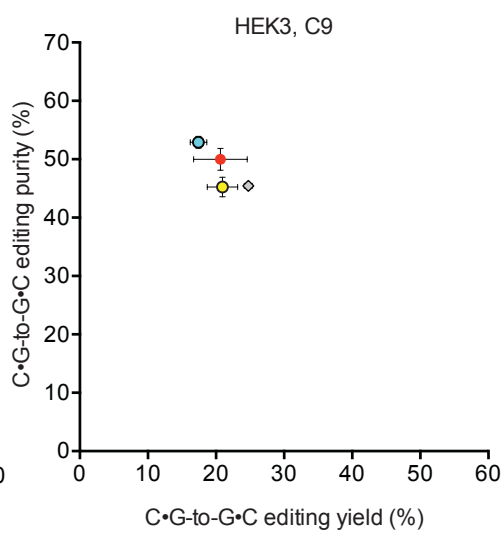
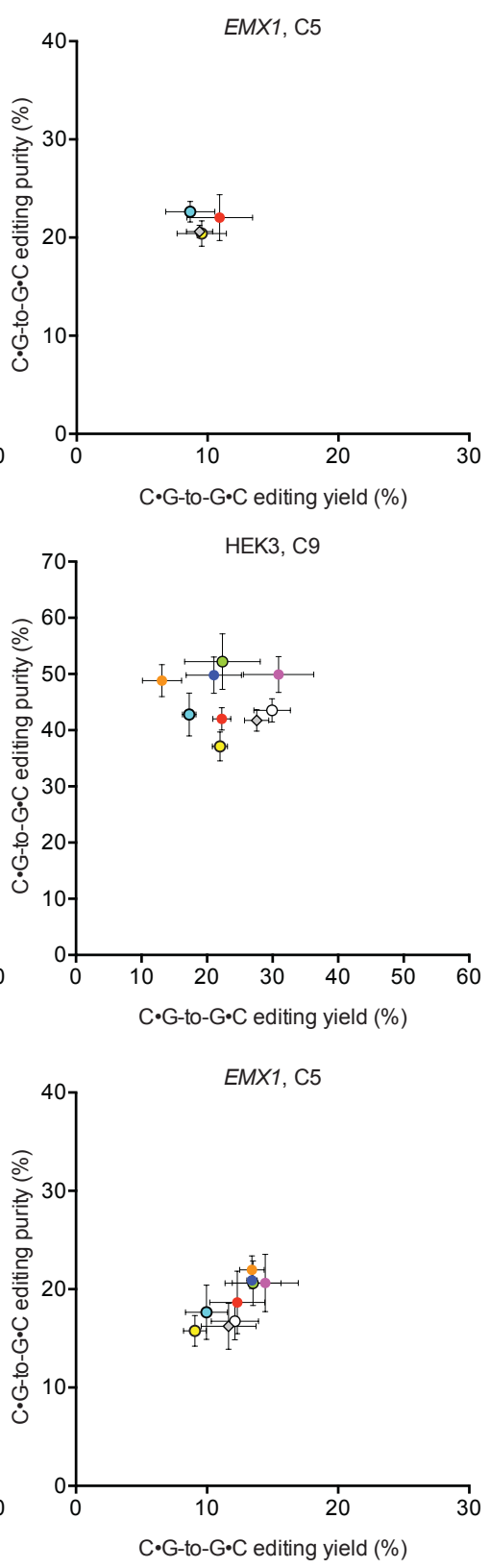
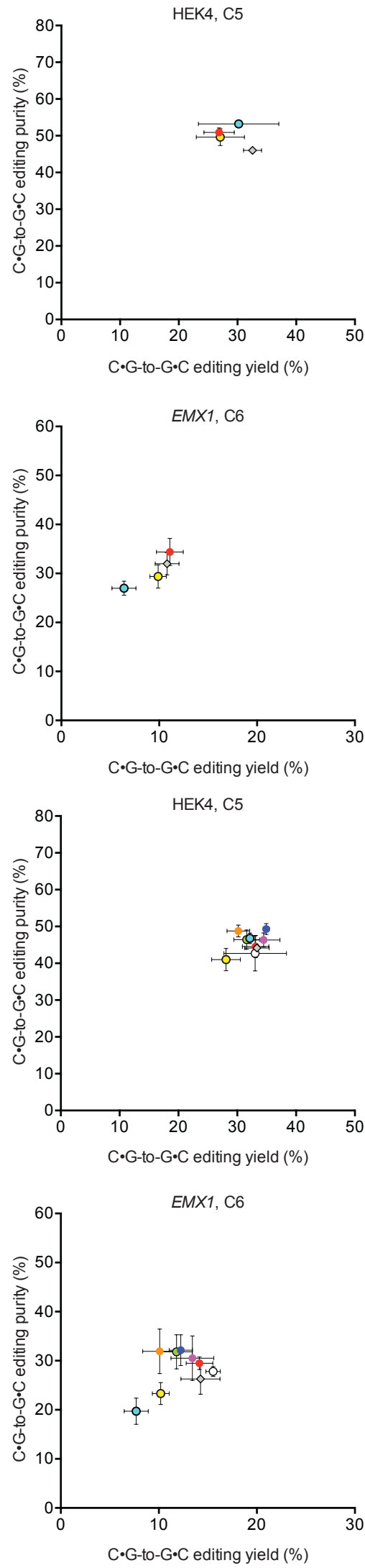

Supplementary Figure 6 . Fusion of proteins to AXC scaffold alters $C \cdot G-$-to-G.C editing outcomes in HEK293T cells. (a) C.G-to-G・C editing outcomes of CGBE candidates containing proteins identified in the screen as $\mathrm{N}$-terminal fusions. (b) $\mathrm{C} \cdot \mathrm{G}$-to-G・C editing 
outcomes of CGBE candidates containing tandem fusion of proteins identified in the screen. $\mathrm{C} \cdot \mathrm{G}$-to-G・C editing yield is shown on the $\mathrm{x}$-axis and product purity is shown on the $\mathrm{y}$-axis. Values and error bars reflect the mean and standard deviation of three biological replicates. Window position annotations indicate the in-window target nucleotides where the SpCas9 PAM is at positions 21-23. HEK2=HEK293T cells site 2; HEK3=HEK293T cells site 3; HEK4=HEK293T cells site 4. Editing efficiencies, purities, and indel profiles for constructs that were tested but were not depicted in this figure can be found in Supplementary Data 1. 

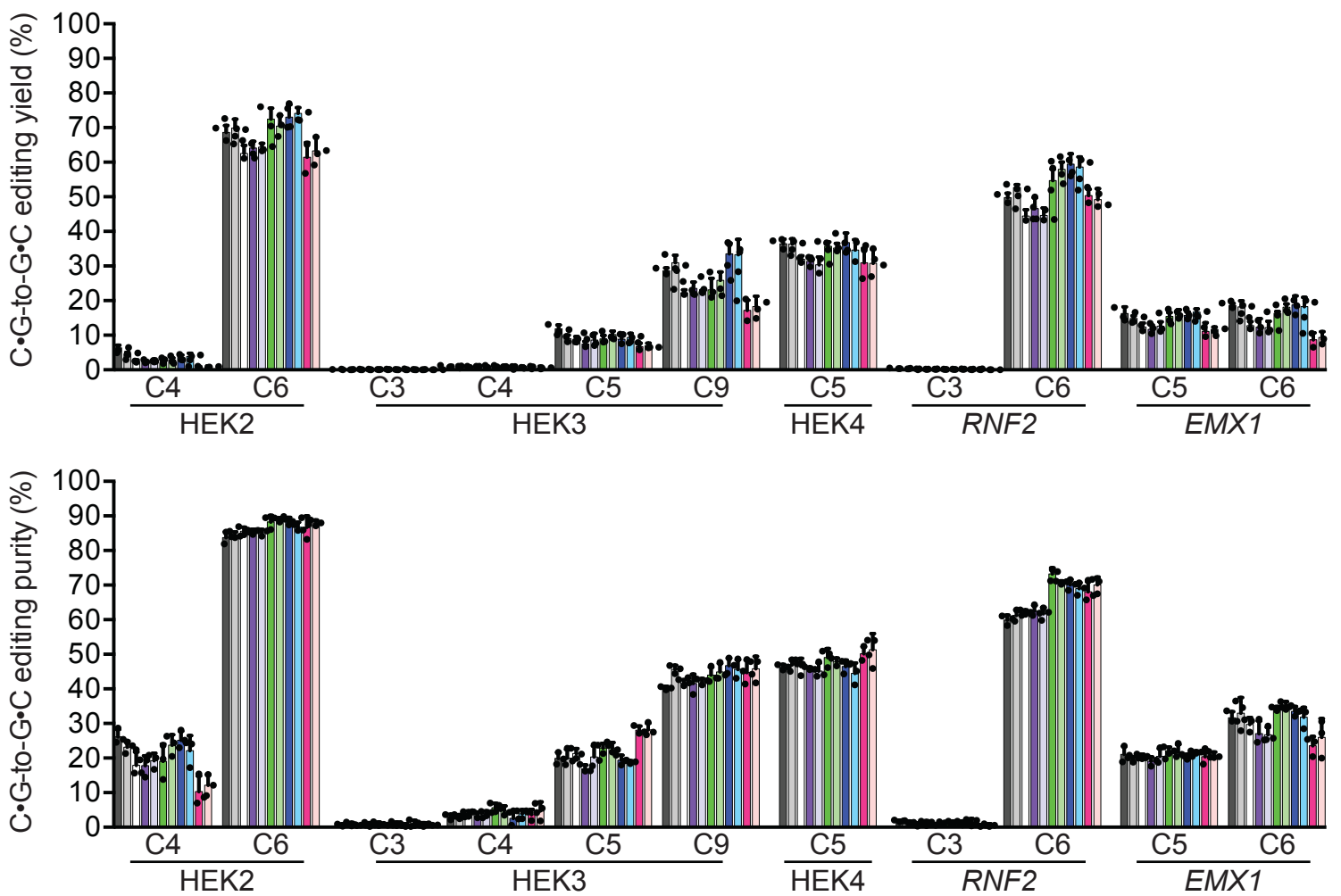
AX-1aa-C
EXO1-32aa-AXC
POLD2-32aa-AXC
UdgX-32aa-AXC $\square$ RBMX-32aa-AXC
$A X-32 a a-C$
EXO1-60aa-AXC $\square$ POLD2-60aa-AXC
UdgX-60aa-AXC
RBMX-60aa-AXC
$A X-60 a a-C$

Supplementary Figure 7. Optimization of linkers between CGBE components. C.G-to$\mathrm{G} \cdot \mathrm{C}$ editing outcomes for CGBE candidates with 1-aa, 32-aa, or 60-aa linkers. Values and error bars reflect the mean and standard deviation of three biological replicates, shown as individual data points. HEK2=HEK293T cells site 2; HEK3=HEK293T cells site 3;

HEK4=HEK293T cells site 4. C4, C6, and similar annotations indicate the in-window target nucleotides where the SpCas9 PAM is at positions 21-23. 

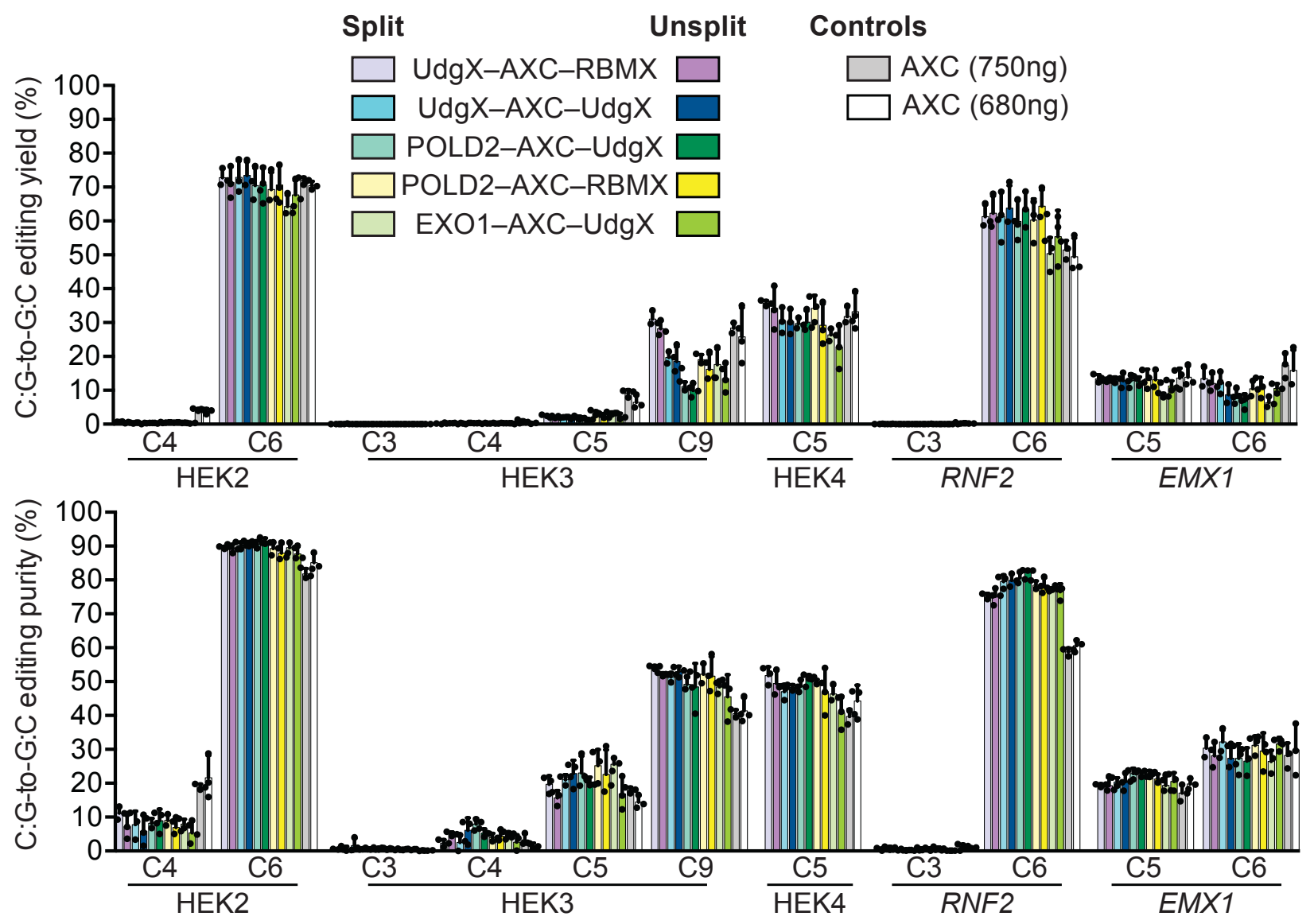

Supplementary Figure 8. Split-intein and unsplit CGBE variants edit with similar yield and product purity. $C \cdot G-$ to-G.C editing outcomes for split-intein (light bars) and unsplit (dark bars) CGBE variants tested in HEK293T cells at five genomic loci. Values and error bars reflect the mean and standard deviation of three biological replicates, shown as individual data points. HEK2=HEK293T cells site 2; HEK3=HEK293T cells site 3; HEK4=HEK293T cells site 4. C4, C6, and similar annotations indicate the in-window target nucleotides where the SpCas9 PAM is at positions 21-23. 


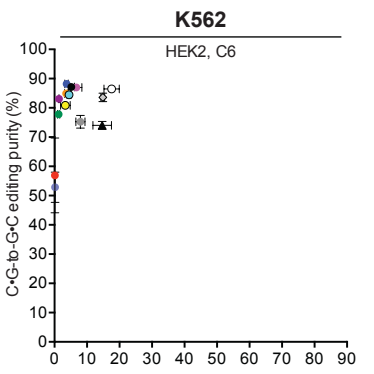

C.G-to-G.C editing yield (\%)
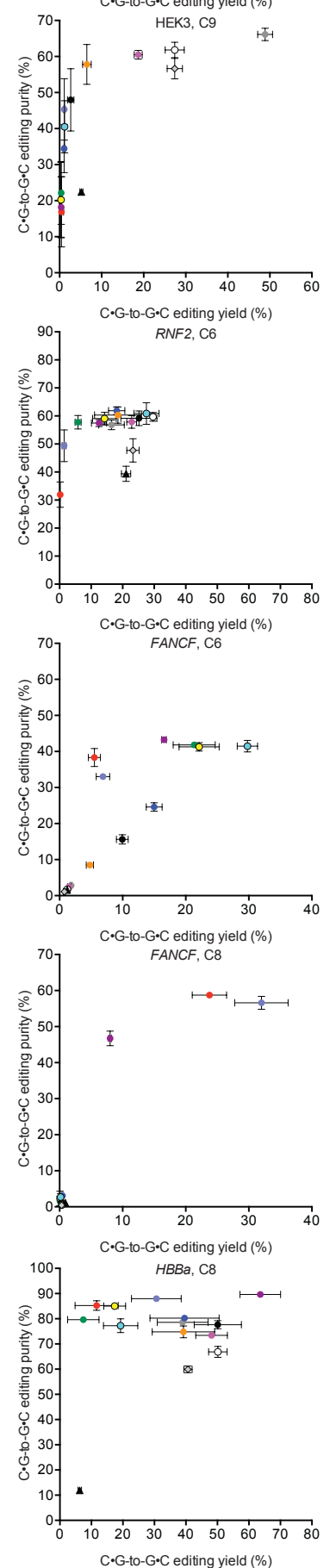

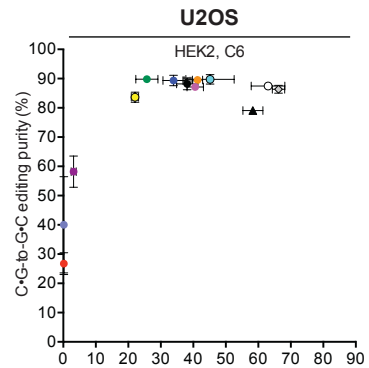

C.G-to-G.C editing yield $(\%)$

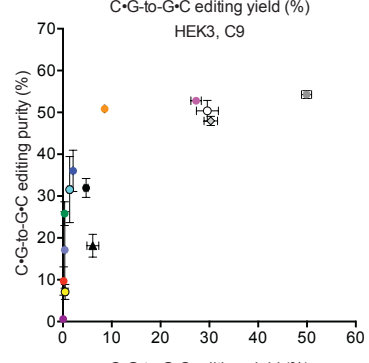

C.G-to-G.C editing yield (\%)

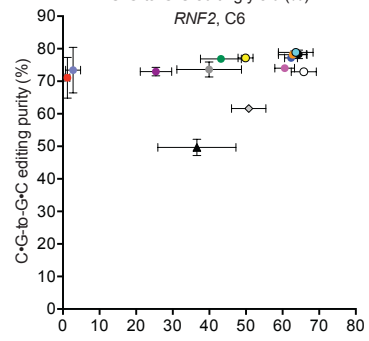

C.G-to-G.C editing yield (\%)
FANCF, C6

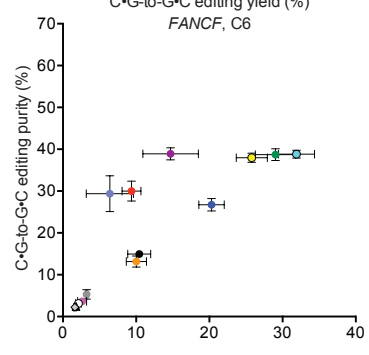

C.G-to-G•C editing yield (\%)
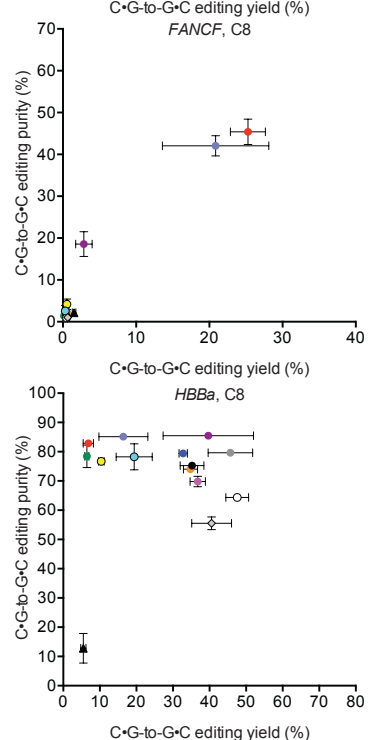

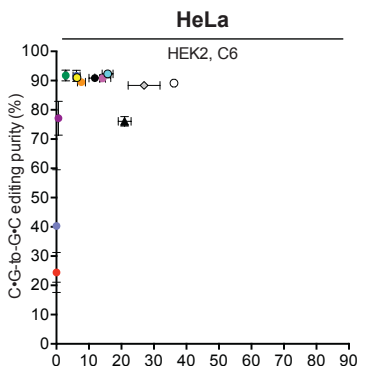

C.G-to-G.C editing yield (\%)
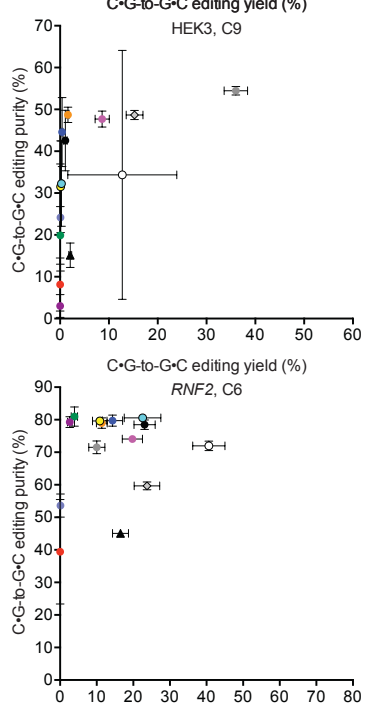

C.G-to-G.C editing yield (\%)
FANCF, C6
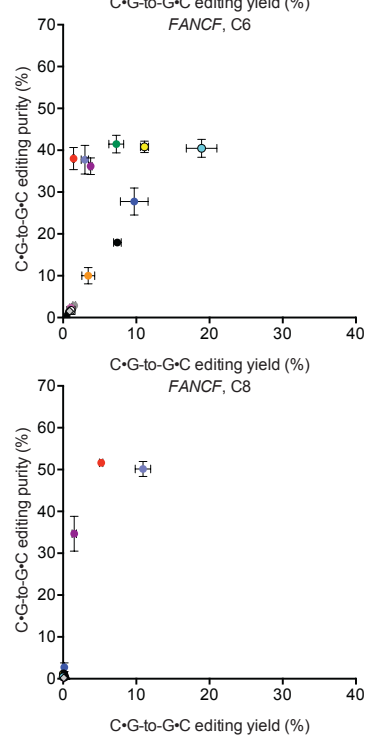

- POLD2-eA3A-UdgX-HF-nCas9-RBMX

- UdgX-Anc689-UdgX-nCas9-UdgX

- UdgX-Anc689-UdgX-nCas9-RBMX

- UdgX-EE-UdgX-nCas9-UdgX

o UdgX-EE-UdgX-nCas9-RBMX

- UdgX-EE-UdgX-nCas9

- POLD2-AXC-UdgX

- UdgX-AXC-RBMX

o UdgX-AXC

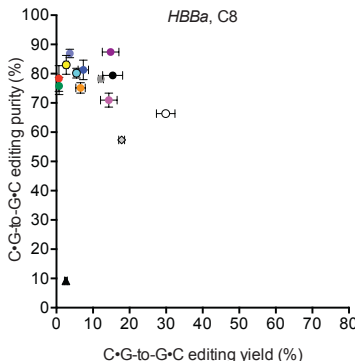

- UdgX-APOBEC1-UdgX-HF-nCas9

- RBMX-eA3A-UdgX-nCas9

- RBMX-eA3A-UdgX-HF-nCas9

$\diamond \mathrm{AXC}$

$\triangle$ APOBEC1-nCas9 (BE4B)

Supplementary Figure 9. Performance of CGBE variants in K562, U2OS, and HeLa cells. C.G-to-G·C editing outcomes in K562 cells (left column), U2OS cells (middle column), and HeLa cells (right column) at six target cytosines across five genomic loci. Editor identities 
are depicted at the bottom of the figure. $\mathrm{C} \cdot \mathrm{G}-\mathrm{to}-\mathrm{G} \cdot \mathrm{C}$ editing yield is shown on the $\mathrm{x}$-axis and product purity is shown on the y-axis. Window position annotations indicate the in-window target nucleotides where the SpCas9 PAM is at positions 21-23. Values and error bars reflect the mean and standard deviation of three biological replicates. HEK2=HEK293T cells site 2; HEK3=HEK293T cells site 3. 

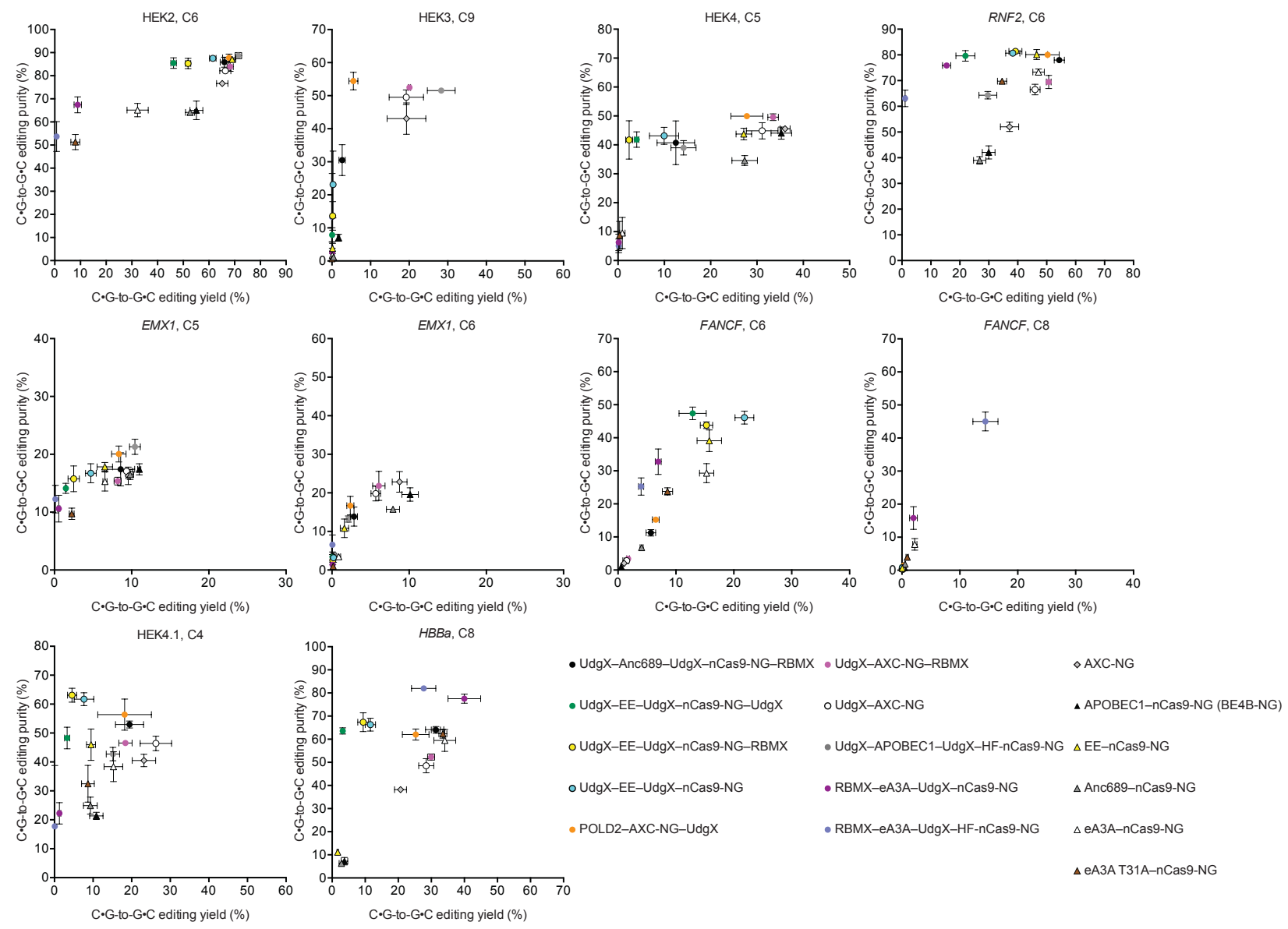

Supplementary Figure 10. CGBE activity using Cas9-NG ${ }^{1}$. C.G-to-G・C editing outcomes in HEK293T cells using CGBE variants containing Cas9-NG at eight target cytosines across seven genomic loci. C.G-to-G・C editing yield is shown on the $\mathrm{x}$-axis and product purity is shown on the y-axis. Values and error bars reflect the mean and standard deviation of three biological replicates. Window position annotations indicate the in-window target nucleotides where the SpCas9 PAM is at positions 21-23. HEK2=HEK293T cells site 2; HEK3=HEK293T cells site 3; HEK4=HEK293T cells site 4; HEK4.1=HEK293T cells site 4.1. 

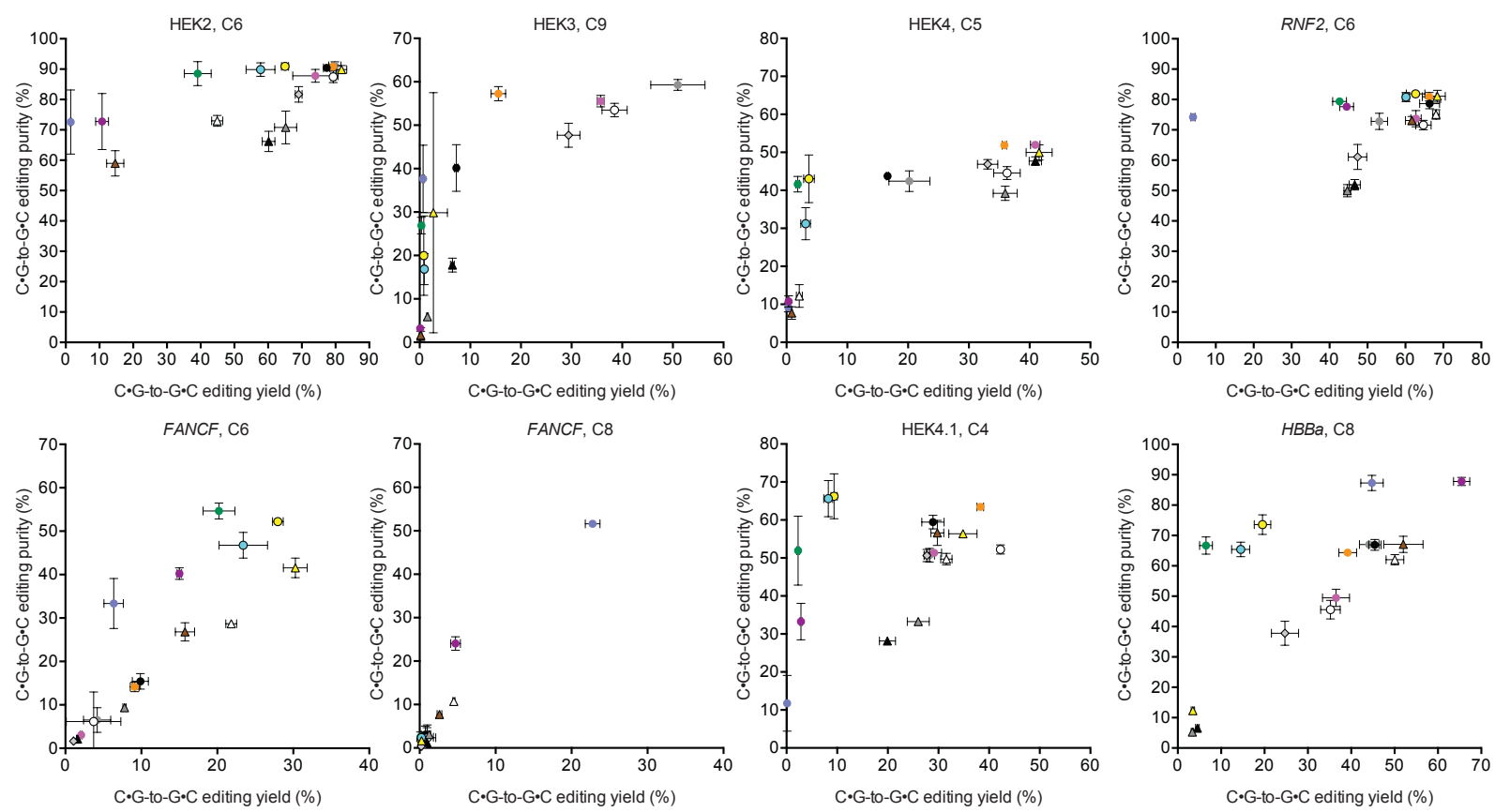

- UdgX-Anc689-UdgX-Cas9-RBMX - UdgX-EE-UdgX-Cas9-UdgX ○ UdgX-EE-UdgX-Cas9 o UdgX-AXC - POLD2-AXC-UdgX - UdgX-APOBEC1-UdgX-HF-Cas9

- RBMX-eA3A-UdgX-Cas9 - UdgX-AXC-RBMX

- RBMX-eA3A-UdgX-Cas9
- RBMX-eA3A-UdgX-HF-Cas

$\begin{array}{ll}\diamond \text { AXC } & \triangle \text { Anc689-Cas9 } \\ \triangle \text { APOBEC1-Cas9 (BE4B) } & \triangle \text { eA3A-Cas9 } \\ \triangle \text { EE-Cas9 } & \triangle \text { eA3A T31A-Cas9 }\end{array}$

Supplementary Figure 11. On-target CGBE editing profiles for off-target analyses. C.Gto-G・C editing outcomes in HEK293T cells using nicking CGBEs at eight target cytosines across seven genomic loci). Editor identities are depicted at the bottom of the figure. C.G-to$\mathrm{G} \cdot \mathrm{C}$ editing yield is shown on the $\mathrm{x}$-axis and product purity is shown on the $y$-axis. Values and error bars reflect the mean and standard deviation of three biological replicates. Window position annotations indicate the in-window target nucleotides where the SpCas9 PAM is at positions 21-23. HEK2=HEK293T cells site 2; HEK3=HEK293T cells site 3; HEK4=HEK293T cells site 4; HEK4.1=HEK293T cells site 4.1. 
a

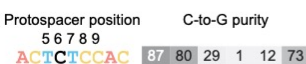

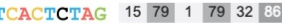
$\begin{array}{lllllllll}\text { AACTACACG } & 88 & 28 & 67 & 14 & 32 & 68\end{array}$ $\begin{array}{llllllll}\text { TCTACACCA } & 91 & 91 & 8 & 67 & 80\end{array}$ ACCACTACG $\quad \begin{array}{lllllll}11 & 42 & 16 & 82 & 75 & 50\end{array}$ $\begin{array}{llllllll}\text { CAGCTAAGG } & 74 & 89 & 38 & 98 & 83 & 69\end{array}$ $\begin{array}{lllllll}7 & & & \end{array}$ TC TITACTG $\begin{array}{lllllll}82 & 86 & 74 & 15 & 46 & 81\end{array}$ GAACTCTAG $22 \quad 7046 \quad 907785$ TCTTTACTG $\begin{array}{llllllll}74 & 86 & 68 & 17 & 33 & 91\end{array}$ AGCTAACTG $84 \quad 12 \quad 73 \quad 0 \quad 11 \quad 77$ $\begin{array}{lllllll}\triangle & & \end{array}$ $\begin{array}{lllllll} & & & \end{array}$ AGGACTACT $\begin{array}{lllllll}70 & 22 & 78 & 4 & 38 & 75\end{array}$ \begin{tabular}{lllllll}
\hline$A G C T A C G G$ & 84 & 76 & 0 & 62 & 75
\end{tabular} $\begin{array}{llllllll}\text { CAGATCTGC } & 28 & 27 & 33 & 87 & 36 & 44\end{array}$ $\begin{array}{lllllll}G \text { CTAGGCCA } & 87 & 81 & 0 & 15 & 8\end{array}$ $\begin{array}{lllllll} & & & 86 & 58 & 23\end{array}$ $\begin{array}{llllllll}\text { AGGACTCTA } & 23 & 75 & 24 & 80 & 70 & 86\end{array}$ $\begin{array}{llllllll}\text { TTACCTCTA } & 19 & 75 & 8 & 62 & 69 & 84\end{array}$ CTGGGAACT $\begin{array}{lllllll}61 & 3 & 70 & 5 & 13 & 35\end{array}$
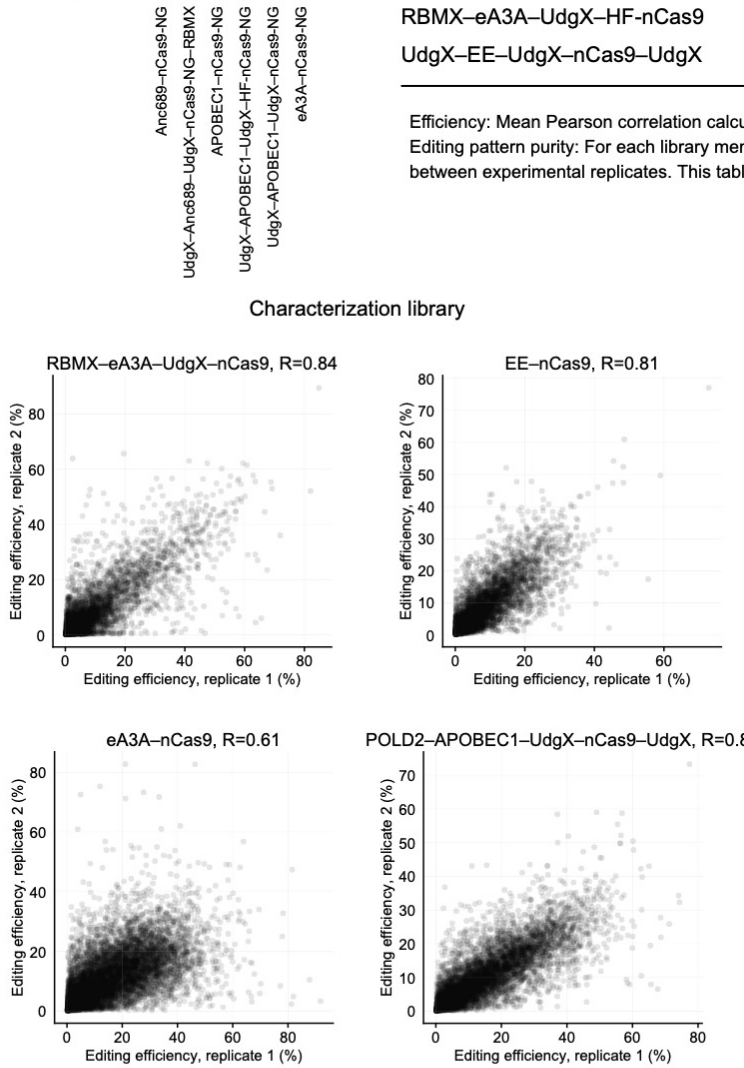

d
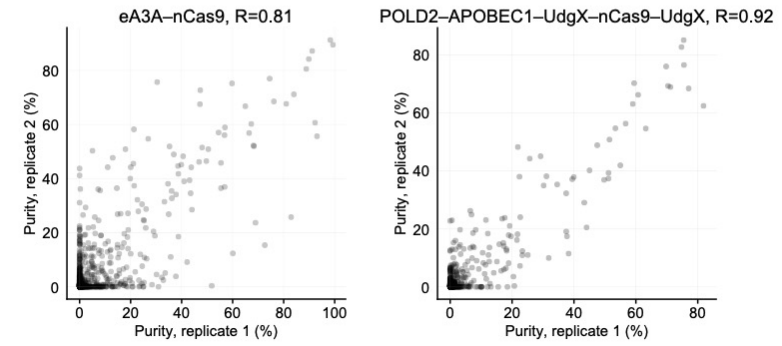

Replicate consistency

\begin{tabular}{llccc}
\hline & \multicolumn{2}{c}{ Characterization library } & \multicolumn{2}{c}{ Transversion library } \\
\cline { 2 - 5 } Editor & Efficiency & $\begin{array}{c}\text { Editing pattern } \\
\text { purity }\end{array}$ & Efficiency & $\begin{array}{c}\text { Editing pattern } \\
\text { purity }\end{array}$ \\
\hline Anc689-nCas9-NG & 0.85 & 0.90 & 0.91 & 0.93 \\
APOBEC1-nCas9-NG & 0.83 & 0.92 & 0.89 & 0.89 \\
eA3A-nCas9-NG & 0.61 & 0.75 & 0.94 & 0.92 \\
eA3A-T31A-nCas9-NG & 0.35 & 0.72 & 0.93 & 0.87 \\
EE-nCas9-NG & 0.81 & 0.94 & - & - \\
UdgX-Anc689-UdgX-nCas9-RBMX & 0.92 & 0.90 & 0.84 & 0.92 \\
POLD2-APOBEC1-UdgX-nCas9-UdgXX & 0.83 & 0.87 & - & - \\
UdgX-APOBEC1-UdgX-nCas9 & - & - & 0.92 & 0.91 \\
UdgX-APOBEC1-UdgX-HF-nCas9 & 0.91 & 0.87 & 0.93 & 0.93 \\
RBMX-eA3A-UdgX-nCas9 & 0.84 & 0.86 & - & - \\
RBMX-eA3A-UdgX-HF-nCas9 & 0.81 & 0.92 & - & - \\
UdgX-EE-UdgX-nCas9-UdgX & 0.84 & 0.92 & - & -
\end{tabular}

Efficiency: Mean Pearson correlation calculated across library members between experimental replicates.

Editing pattern purity: For each library member, a Pearson correlation was calculated across editing pattern purities

between experimental replicates. This table reports the average Pearson correlation across library members.
Transversion library
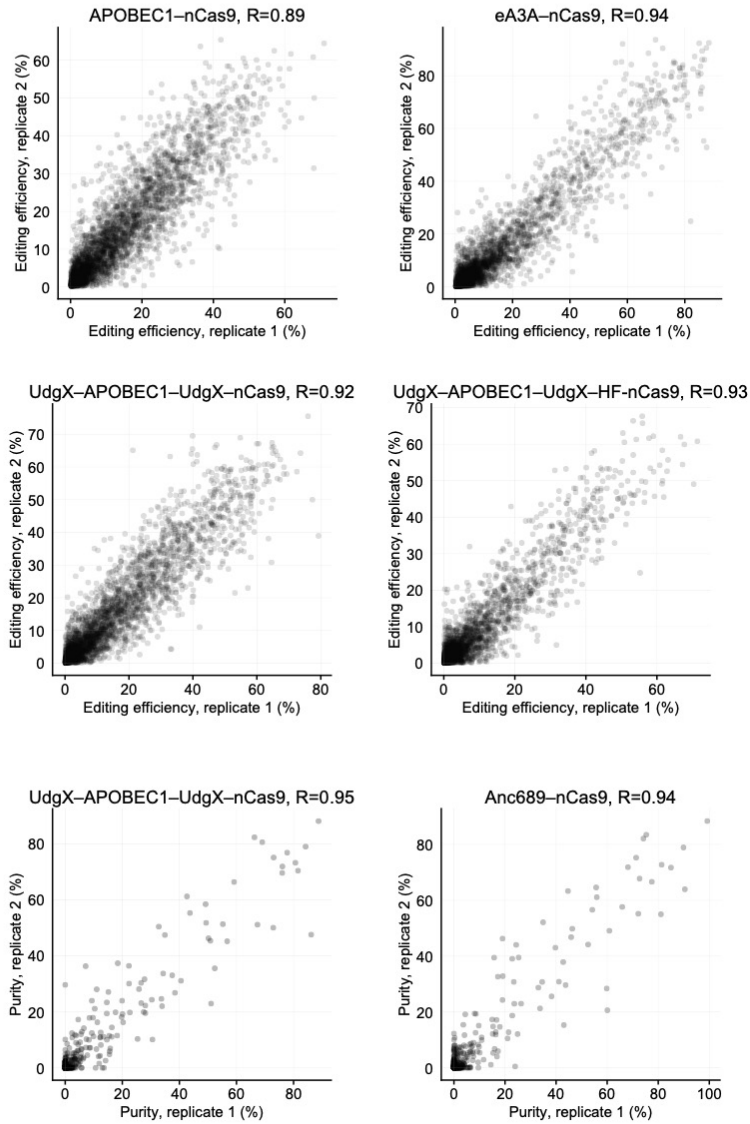

Supplementary Figure 12. Transversion-enriched SNV library analysis. (a) Heatmap of observed C.G-to-G.C purities by CGBE variants in target contexts from the transversionenriched SNV library in mES cells. Black nucleotides indicate the cytosine for which purity is calculated. Target sites were sorted by outcome variance and manually selected. (b) 
Replicate consistency statistics. (c) Scatter plots of base editing efficiency between experimental replicates. Each point represents a single target site. (d) Scatter plots of editing purities between experimental replicates. Each point represents a unique editing pattern in a target site. Scatter plot is plotted across 30 library members. 

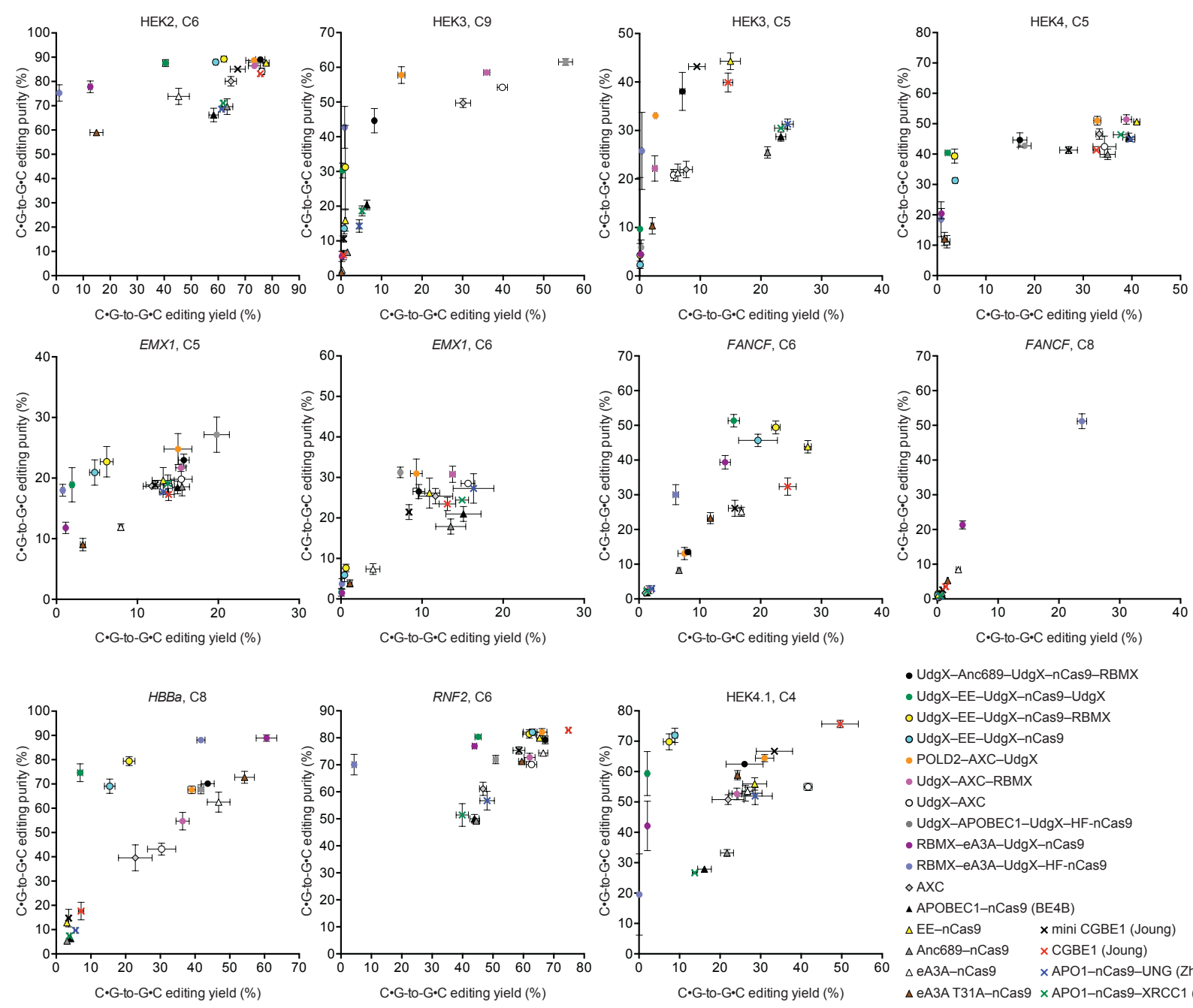

- UdgX-Anc689-UdgX-nCas9-RBMX

- UdgX-EE-UdgX-nCas9-UdgX

o UdgX-EE-UdgX-nCas9-RBMX

- UdgX-EE-UdgX-nCas9

- POLD2-AXC-UdgX

- UdgX-AXC-RBMX

O UdgX-AXC

- UdgX-APOBEC1-UdgX-HF-nCas9

- RBMX-eA3A-UdgX-nCas9

- RBMX-eA3A-UdgX-HF-nCas 9

$\triangle A X C$

$\triangle$ APOBEC1-nCas9 (BE4B)

$\triangle E E-n$ Cas9 $\quad x$ mini CGBE1 (Joung)

$\triangle$ Anc689-nCas9 $\times$ CGBE1 (Joung)

$\triangle$ eA3A-nCas9 $\times$ APO1-nCas9-UNG (Zhang)

$\triangle$ eA3A T31A-nCas9 $\times$ APO1-nCas9-XRCC1 (Chew)

\section{Supplementary Figure 13. Comparison of CGBEs developed in this study with recently} described CGBEs. C.G-to-G・C editing outcomes for CGBEs reported in this study compared with that of mini CGBE1 ${ }^{2}, \mathrm{CGBE}^{2}, \mathrm{APO} 1-\mathrm{nCas9-UNG^{3 }}$, and APO1-nCas9-XRCC1 ${ }^{4}$ at 11 different target cytidines across eight genomic loci. $C \cdot G$-to-G・C editing yield is shown on the $x$-axis and product purity is shown on the $y$-axis. Values and error bars reflect the mean and standard deviation of three biological replicates. Window position annotations indicate the inwindow target nucleotides where the SpCas9 PAM is at positions 21-23. HEK2=HEK293T cells site 2; HEK3=HEK293T cells site 3; HEK4=HEK293T cells site 4; HEK4.1=HEK293T cells site 4.1 . 

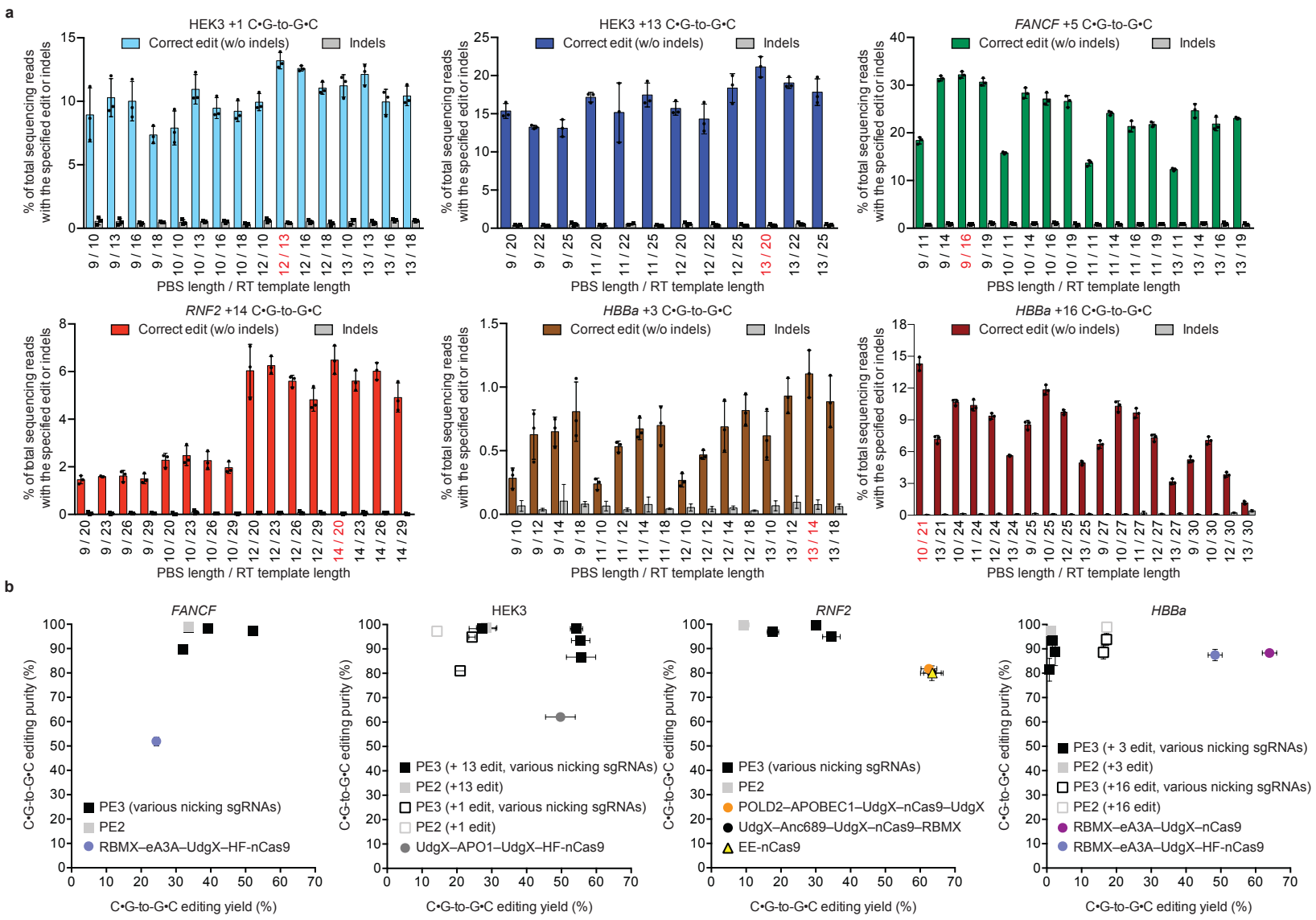

Supplementary Figure 14. Comparison of prime editing and CGBE editing outcomes. (a) C.G-to-G.C editing outcomes in HEK293T cells using PE2 to identify the best-performing pegRNA to make six different edits at four genomic loci (HEK site 3, FANCF, RNF2, and $H B B a$ ) (b) Comparison of CGBE variants with PE2 and PE3 prime editors at four genomic loci. PE3 prime editors use an additional sgRNA to nick the non-edited DNA strand. Values and error bars reflect the mean and standard deviation of three biological replicates. C.G-to$\mathrm{G} \cdot \mathrm{C}$ editing yield is shown on the $\mathrm{x}$-axis and product purity is shown on the $y$-axis in (b). HEK3=HEK site 3. C4, C6, and similar annotations indicate the in-window target nucleotides where the SpCas9 PAM is at positions 21-23. 

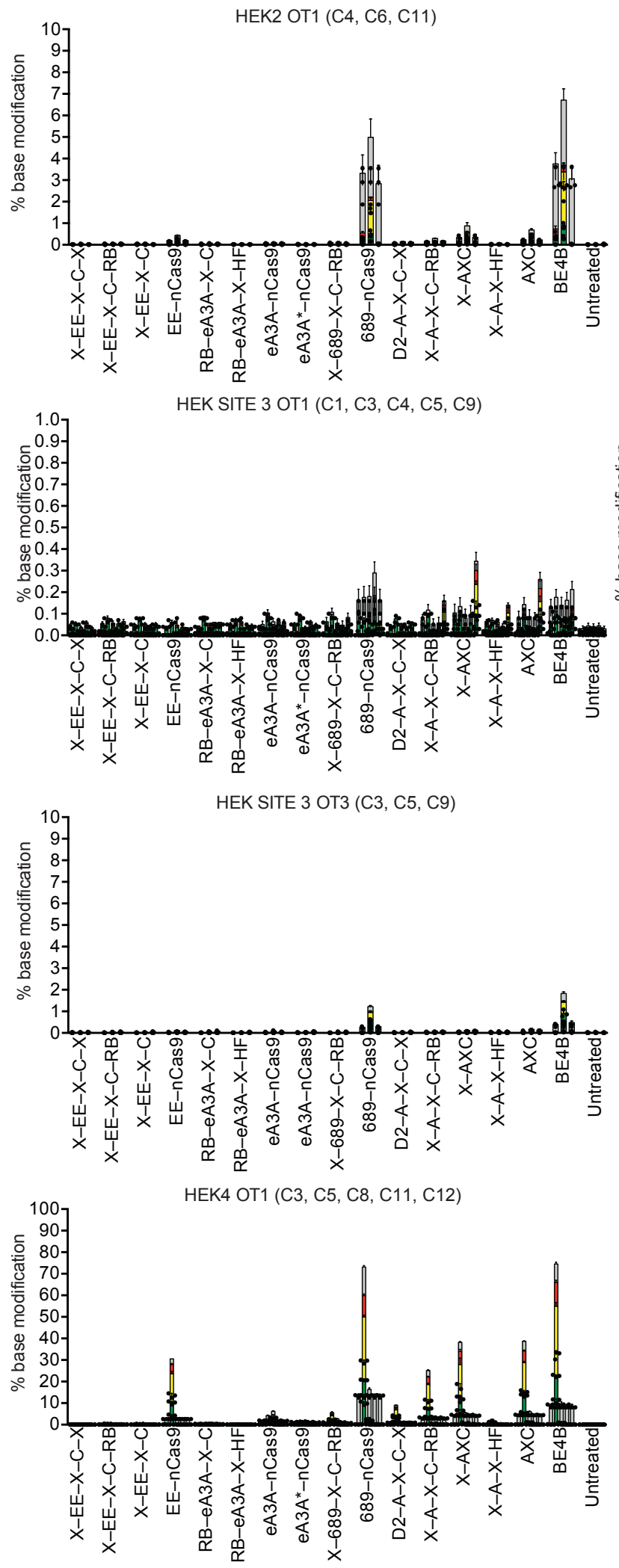

HEK2 OT2 (C4, C11)

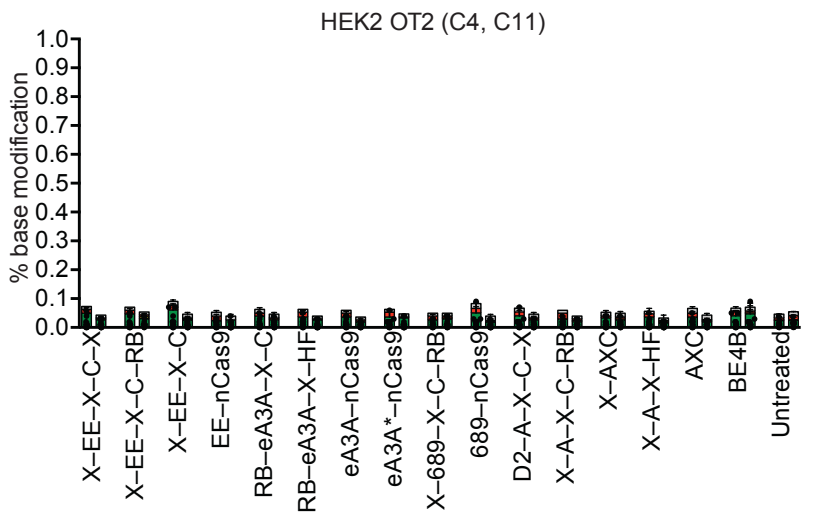

HEK SITE 3 OT2 (C3, C5, C9)

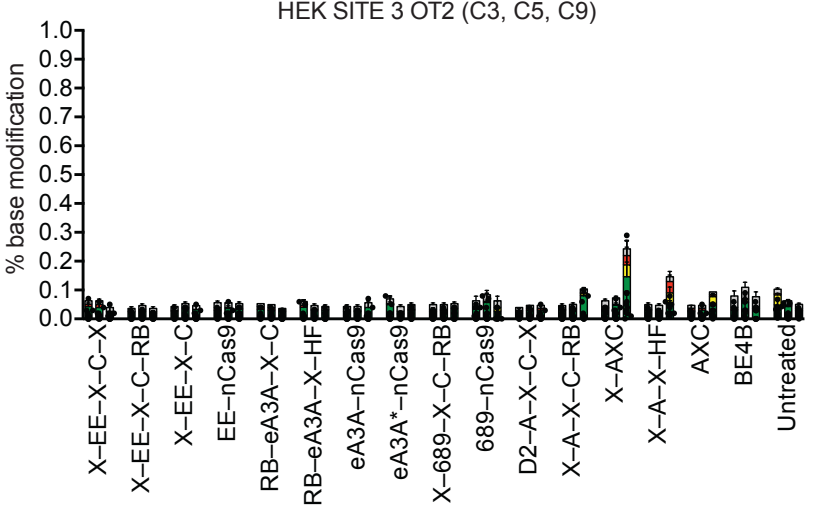

HEK SITE 3 OT4 (C4, C5, C9)

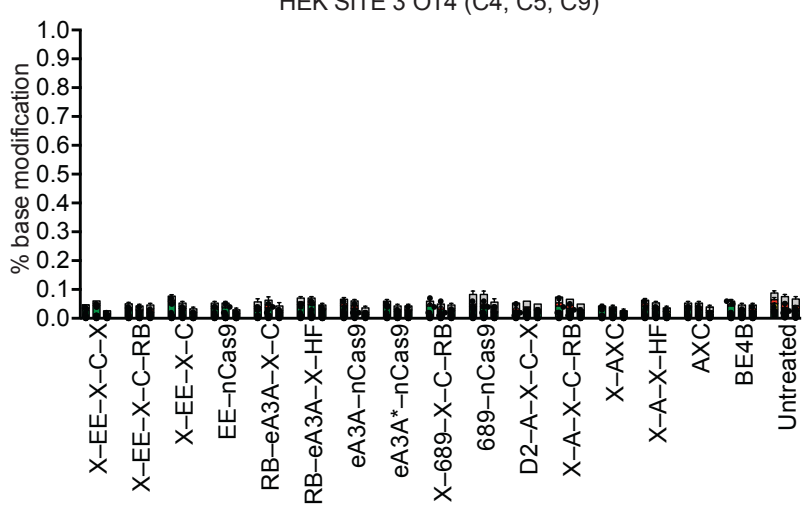

HEK4 OT3 (C3, C5, C8, C11)

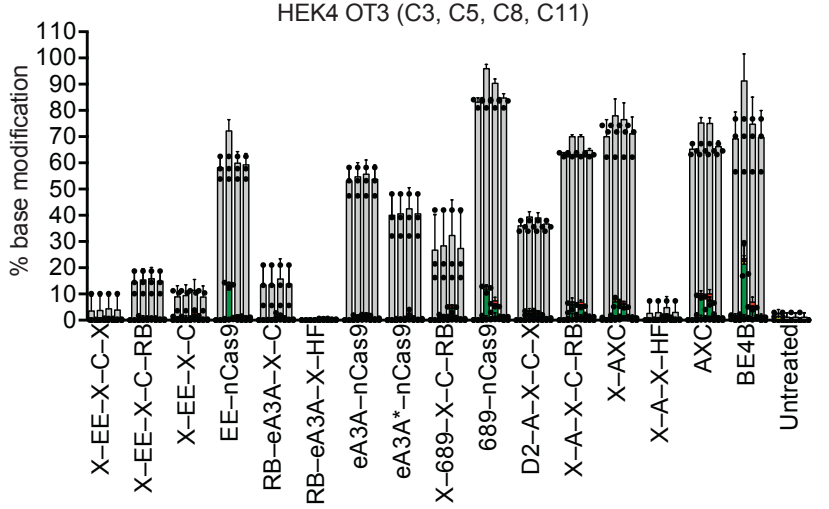



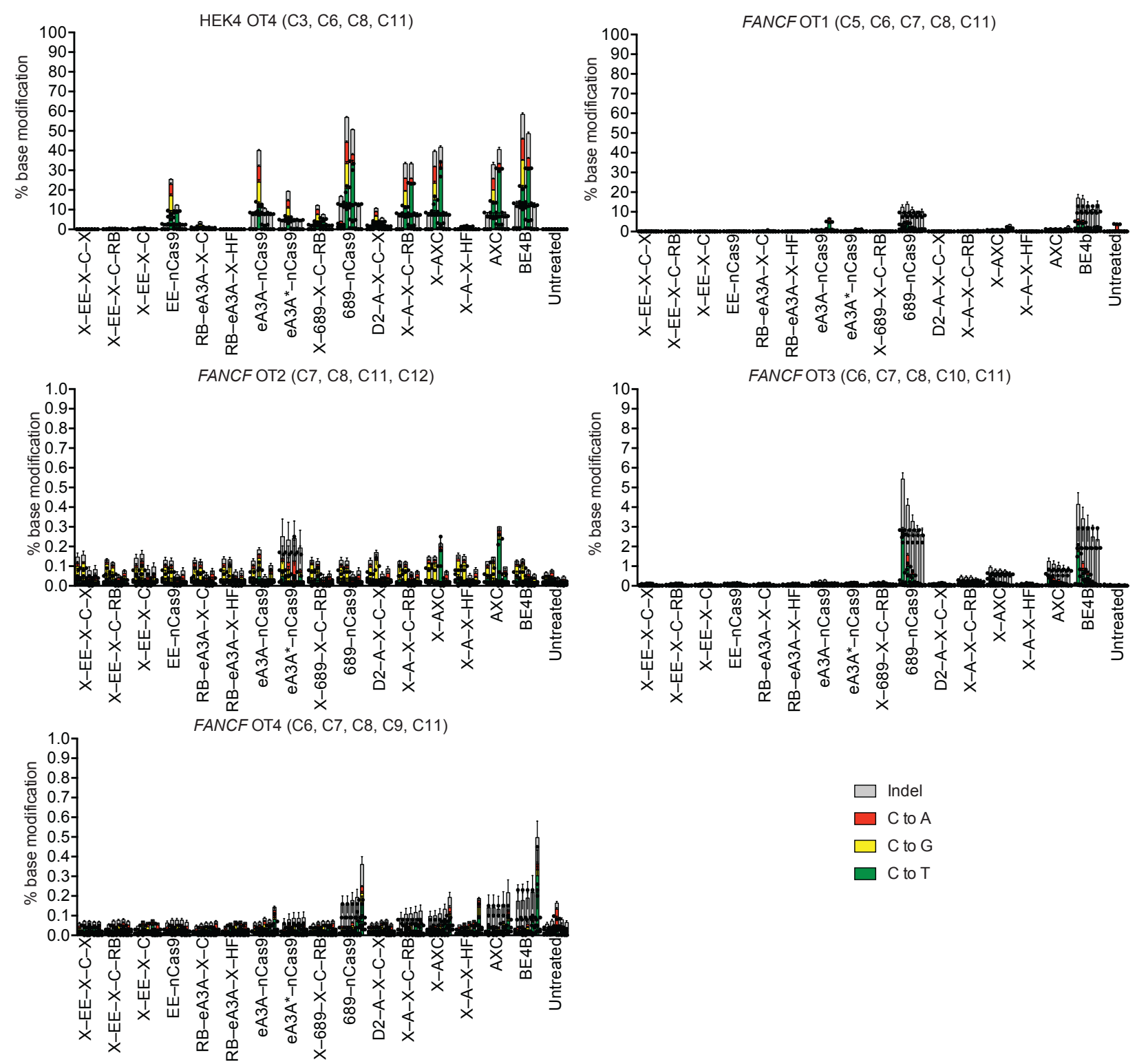

Supplementary Figure 15. Off-target DNA editing activities of CGBEs. CGBE activity at 13 off-target loci. Values and error bars reflect the mean and standard deviation of three biological replicates. HEK2=HEK293T cells site 2; HEK3=HEK293T cells site 3; HEK4=HEK293T cells site 4. X=UdgX, D2=POLD2, RB=RBMX, 689=Anc689, HF=HFnCas9, eA3A $*$ eA3A T31A. 


\section{Supplementary Discussion 1}

We previously demonstrated that the purity of canonical C $\cdot G$-to-T•A edits by CBEs improves dramatically in cells lacking nuclear uracil DNA N-glycosylase (UNG) or when one or more uracil glycosylase inhibitor proteins (UGI) are appended $\mathrm{CBEs}^{5-8}$, suggesting that excision of uracil from genomic DNA to form an abasic site is an important early step in achieving transversion base editing outcomes. Motivated by these insights, we sought to better understand the molecular mechanisms that transform abasic sites into transversion edits in mammalian cells.

We tested whether UdgX fusion proteins require cellular UNG to install C.G-to-G・C edits. C.G-to-G・C editing with AXC was minimal in UNG2 ${ }^{-}$HAP1 cells compared to UNG ${ }^{+}$ cells, confirming that $C \cdot G-$ to-G.C transversion outcomes indeed are promoted by cellular UNG-mediated formation of an abasic site intermediate, even when using the AXC construct (Supplementary Fig. 1a).

AP endonuclease-1 (APE1 or APEX1) initiates short patch base excision repair (spBER) following abasic site formation by nicking the abasic site-containing strand. Polymerases such as PolB then resynthesize the damaged strand using the intact stand as a template $^{9,10}$. We asked whether loss of APE1 could bias the repair of CBE-induced abasic sites towards $\mathrm{C} \cdot \mathrm{G}-\mathrm{to}-\mathrm{G} \cdot \mathrm{C}$ outcomes by measuring cytosine base editing outcomes with nonnicking BE1 (bpNLS-APOBEC1-dead Cas9-bpNLS), nicking BE4B (bpNLS-APOBEC1Cas9 D10A-bpNLS), and the AXC construct in APE1-deficient HAP1 cells. We observed no meaningful differences in editing by BE1 in APE1-deficient HAP1 cells compared to APE1 ${ }^{+}$ HAP1 cells. C.G-to-G.C editing yields with either BE4B or AXC were modestly increased in $A P E 1^{-}$cells compared to $A P E 1^{+}$cells and $\mathrm{C} \cdot \mathrm{G}-\mathrm{to}-\mathrm{G} \cdot \mathrm{C}$ editing purity was not significantly different (Supplementary Fig. 1b). These data suggest that APE1 does not play a major non-redundant role in resolving CBE edits towards transversion outcomes.

Next, we evaluated the contributions of mismatch repair proteins on $\mathrm{C} \cdot \mathrm{G}-\mathrm{to}-\mathrm{G} \cdot \mathrm{C}$ editing outcomes ${ }^{11}$. Using the same panel of BE1, BE4B, and AXC editors, we observe only modest changes in $\mathrm{C} \cdot \mathrm{G}-\mathrm{to}-\mathrm{G} \cdot \mathrm{C}$ editing yield and no significant changes in editing purity in $\mathrm{MLH} 1^{-} \mathrm{HAP} 1$ cells compared with $\mathrm{MLH}^{+}$controls (Supplementary Fig. 1c).

Surprisingly, loss of REV1- a cellular polymerase known for its deoxycytidyl transferase activity ${ }^{12,13}$ - modestly increased, rather than decreased, C.G-to-G・C editing outcomes. These data suggest that alternative polymerases could install $\mathrm{C}$ opposite abasic lesions that result from cytosine base editing. (Supplementary Fig. 1d). To explore the possibility that other polymerases may play key roles in installing either the $\mathrm{C}$ opposite the abasic site or the $\mathrm{G}$ that replaces the original $\mathrm{C}$, we constructed a panel of ten $\mathrm{N}$ - and $\mathrm{C}$ terminal fusions of DNA polymerase catalytic domains to the AXC construct and assessed editing outcomes at three genomic loci in HEK293T cells. We did not observe any

consistently improved editing outcomes with any polymerase-fused AXC variant ${ }^{10,14}$ (Supplementary Fig. 2).

\section{Supplementary Discussion 2}


Using screening data, we identified genes that control the base editing activity window. For each CRISPRi sgRNA, we calculated the fraction of all edited reads that included a point mutation at each position in or near the target sequence. We then identified genes that significantly changed the relative editing frequency at any nucleotide position compared to non-targeting CRISPRi sgRNA controls (Supplementary Figs 4c). Intriguingly, two helicase genes, RECQL and HLTF, emerged from this analysis. Repression of RECQL selectively reduced editing at the PAM-distal $C$ in position +1 of the target sequence, where the SaCas9 NNGRRT PAM is positions 22-27 (Supplementary Fig. 5), while repression of HLTF specifically increased editing at the $G$ in position +3 (Supplementary Fig. 5). Together, these observations suggest that cellular helicases can influence the location of base editing activity within a target sequence, potentially by increasing the accessibility of cytosines at position +1 in the case of RECQL, or by reducing accessibility of the $C$ opposite the position $+3 \mathrm{G}$ in the case of $H L T F$.

\section{Supplementary Discussion 3}

We used CGBE-Hive to identify disease-relevant C.G-to-G.C SNVs that could be installed in HEK293T cells using CGBEs characterized in this study. The CTNNB1 c.2138 -1 G>C mutation, a cancer-associated allele, was installed by UdgX- APOBEC1-UdgX-HFnCas9 with higher yield $(64 \pm 1.0 \%$ vs. $51 \pm 0.5 \%)$ and purity $(75 \pm 0.8 \%$ vs. $67 \pm 1.5 \%)$ than the best-performing simple deaminase-nCas9 fusion, Anc689-nCas9 (Fig. 6f). Additionally, the DIS3L2 c.2011 -1 G>C mutation, associated with Perlmen Syndrome, was installed with higher purity by UdgX-Anc689-UdgX-nCas9-RBMX (46 $\pm 1.1 \%$ vs. $41 \pm 1.3 \%)$ and similar editing efficiency ( $32 \pm 2.4 \%$ vs. $31 \pm 2.3 \%$ ) compared to the best-performing deaminasenCas9, eA3A-nCas9 (Fig. 6f). We also used NG-CGBEs to install a pathogenic SNV in the KCNQ2 gene predicted to be editable by CGBE-Hive with RBMX-eA3A-UdgX-nCas9, and observed $37.5 \pm 3.3 \%$ yield and $79.5 \pm 1.0 \%$ purity (Fig. 6 f). These results indicate that CGBEs using both wild-type $\mathrm{nCas} 9$ and a Cas9 variant engineered to be compatible with non-native PAM sequences can efficiently install disease-associated alleles in human cells as predicted by CGBE-Hive. These results collectively demonstrate that the CGBEs developed in this study can install disease relevant SNPs with high efficiency and purity.

\section{Supplementary Discussion 4}

We compared the editing activity of CGBEs developed in this study and previously described CGBEs ${ }^{2-4}$ (mini CGBE1, CGBE1, APOBEC1-nCas9-UNG, and APOBEC1nCas9-XRCC1) across eight genomic loci in HEK293T cells. The CGBEs developed in this study outperform previously described CGBEs at six of eight tested loci, with the broader sequence substrate scope of the CGBEs described in this work enabling efficient editing at a broader array of loci. For example, at HEK site $3 \mathrm{C}$, UdgX-APOBEC1-UdgX-HF-nCas9 edits with $55.4 \pm 1.1 \%$ yield and $61.5 \pm 0.9 \%$ purity while the best previous CGBE (APOBEC1nCas9-XRCC1) edits with $5.22 \pm 0.3 \%$ yield and $18.7 \pm 1.4 \%$ purity (Supplementary Fig. 13). Additionally, at $\mathrm{HBBa} \mathrm{C} 8, \mathrm{RBMX}-\mathrm{eA} 3 \mathrm{~A}-\mathrm{UdgX}-\mathrm{C}$ edits with $60.6 \pm 3.0 \%$ yield and $88.9 \pm 1.4 \%$ purity while the best performing previous CGBE (CGBE1; eUNG-APOBEC1 R33A-nCas9) edits with $7.2 \pm 0.8 \%$ yield and $17.6 \pm 3.7 \%$ purity (Supplementary Fig. 13). At the two sites, 
RNF2 and HEK4.1 that were very well edited by deaminase-nCas9 constructs, the CGBEs in this study performed comparably or modestly worse than the best previously reported CGBE. For RNF2, editing purity was comparable for CGBE1 and POLD2-APOBEC1-UdgX-nCas9UdgX (CGBE1 $=82.8 \pm 0.9 \%$ vs. $82.1 \pm 1.4 \%$ ) while yield improved to $74.8 \pm 0.4 \%$ for CGBE1 vs. $66.1 \pm 1.6 \%$ (Supplementary Fig. 13). At HEK4.1, editing yield and purity for CGBE1 were $49.6 \pm 4.5 \%$ and $75.7 \pm 1.2 \%$, respectively, compared with $41.7 \pm 1.0 \%$ and $55.0 \pm 1.2 \%$ for UdgX-APOBEC1-UdgX-nCas9 (Supplementary Fig. 13).

\section{Supplementary Discussion 5}

Recently described prime editors (PEs) consist of Cas9 nickase fused to an engineered reverse transcriptase ${ }^{15,16}$. PEs are targeted to a genomic locus by an engineered prime editing guide RNA (pegRNA) that encodes both the desired edit and the target site. Since prime editing enables a broad range of genome edits including all 12 possible singlebase conversions, as well as small insertions and deletions ${ }^{15,16}$, we sought to characterize how CGBEs and prime editors compare.

Successful prime editing requires thorough optimization of the primer binding site (PBS) and the reverse transcriptase template in the pegRNA ${ }^{15,16}$. We optimized these parameters for C.G-to-G・C edits at four genomic loci (FANCF, HEK site 3, RNF2, and HBBa) (Supplementary Fig. 14a). We then tested each of these optimized pegRNAs using PE2, which does not nick the non-edited strand, as well as PE3, which nicks the non-edited strand by adding an additional sgRNA. We also evaluated the best-performing CGBE for these loci and compared editing efficiencies and product purities of CGBEs and PEs at these loci.

Two of the four loci (HEK site 3 and FANCF) were edited with higher efficiency and purity using PE compared with CGBEs. The best PE-mediated editing of the FANCF locus was $52.3 \pm 0.8 \%$ yield with $97.3 \pm 0.7 \%$ purity with $P E 3$, while the best CGBE-mediated editing (with RBMX-eA3A-UdgX-HF-nCas9) provided $24.4 \pm 0.6 \%$ yield and $52.7 \pm 2.8 \%$ purity. Likewise, the best balance of editing yield and purity by PE at the HEK site 3 locus was $54.3 \pm 1.8 \%$ yield with $98.2 \pm 0.1 \%$ purity with PE3, while the best CGBE editing (UdgXAPOBEC1-UdgX-HF-nCas9) was $49.7 \pm 4.3 \%$ yield and $62.1 \pm 0.7 \%$ purity. At the other two loci (RNF2 and $\mathrm{HBBa}$ ), however, the best-performing CGBEs characterized in this work provide the desired edits with higher efficiency than PE (Supplementary Fig. 14b). At the RNF2 locus PE3 installed the target nucleotide with $34.5 \pm 2.5 \%$ yield and $94.8 \pm 1.0 \%$ purity while CGBE (POLD2-APOBEC1-UdgX-C-UdgX) installed the same mutation with $62.5 \pm 2.3 \%$ yield and $81.7 \pm 1.7 \%$ purity. $H B B a$ editing by $P E$ proceeded with $17.2 \pm 1.1 \%$ yield and $98.9 \pm 0.63 \%$ purity with PE2 (slightly outperforming PE3) while CGBE (RBMX-eA3AUdgX-C) edited with $64.0 \pm 2.1 \%$ yield and $88.3 \pm 1.6 \%$ purity (Supplementary Fig. 14b).

\section{Supplementary Discussion 6}

We examined CGBE off-target editing activity at thirteen off-target loci for four sgRNAs (HEK site 2, HEK site 3, HEK site 4, and FANCF). On-target editing efficiency was confirmed and is shown in Supplementary Fig. 11. While off-target editing varied by site, as has been reported previously ${ }^{17}$, the deaminase domain is was primary determinant of off-target editing activity. Across all cytidines assessed within a broadened search window (protospacer 
positions $\mathrm{C} 1-\mathrm{C} 12)$ to capture all possible off-target edits, we observed an average off-target nucleotide modification frequency of $5.9 \pm 0.5 \%$ for eA3A-nCas9, $6.4 \pm 0.3 \%$ for EE-nCas9, $11.9 \pm 0.9 \%$ for APOBEC $1-n C a s 9$, and $13.0 \pm 0.3 \%$ for Anc689-nCas9 (Supplementary Fig. 15). Importantly, the average frequency of off-target in-window editing (any $C \cdot G$ to $T \cdot A, A \cdot T$, $G \cdot C$, or indel at an in-window off-target cytosine) across the thirteen studied off-target loci was substantially decreased for our engineered CGBE variants tested compared to the corresponding simple deaminase-nCas9 fusions (Supplementary Fig. 15). For example, RBMX-eA3A-X-C showed a 4.5-fold reduction in off-target editing compared to eA3AnCas9, while the RBMX-eA3A-X-HF construct, which has a slightly shifted editing window, showed a large 52-fold reduction relative to eA3A-nCas9. Among the 16 characterized CGBE variants containing protein fusions made in this study, off-target editing levels on average were 11.3-fold lower than the corresponding deaminase-nCas9.

Together, these results indicate that the novel protein fusion CGBEs developed in this study offer lower Cas9-dependent off-target editing compared to corresponding CGBEs lacking those fusions, despite their generally higher on-target editing, perhaps because the more complex fusions introduce additional conformational requirements in editor:DNA complexes that are not met by some off-target loci.

Base editor off-target activity may also arise in a sgRNA-independent manner. Such edits are predominantly driven by the deaminase component; therefore, we anticipate that sgRNA-independent off-target activity of CGBE will mirror that of the CBEs that use the same cytosine deaminase. While overexpression of fusion proteins, including DNA repair proteins, as CGBE-components may result in additional sgRNA-independent off-target effects, these are likely to differ, perhaps due to cell-type specific DNA repair profiles, and are therefore best assessed per application. 


\section{Supplementary Sequences}

Deaminase domains

\section{IAPOBEC $1^{6}$}

MSSETGPVAVDPTLRRRIEPHEFEVFFDPRELRKETCLLYEINWGGRHSIWRHTSQNTNKHV EVNFIEKFTTERYFCPNTRCSITWFLSWSPCGECSRAITEFLSRYPHVTLFIYIARLYHHADPR NRQGLRDLISSGVTIQIMTEQESGYCWRNFVNYSPSNEAHWPRYPHLWVRLYVLELYCIILG LPPCLNILRRKQPQLTFFTIALQSCHYQRLPPHILWATGLK*

\section{EE (rAPOBEC1 R126E, R132E) $)^{18}$}

MSSETGPVAVDPTLRRRIEPHEFEVFFDPRELRKETCLLYEINWGGRHSIWRHTSQNTNKHV EVNFIEKFTTERYFCPNTRCSITWFLSWSPCGECSRAITEFLSRYPHVTLFIYIARLYHHADPE NRQGLEDLISSGVTIQIMTEQESGYCWRNFVNYSPSNEAHWPRYPHLWVRLYVLELYCIILG LPPCLNILRRKQPQLTFFTIALQSCHYQRLPPHILWATGLK*

YE1 (rAPOBEC1 W90Y, R126E) ${ }^{18}$

MSSETGPVAVDPTLRRRIEPHEFEVFFDPRELRKETCLLYEINWGGRHSIWRHTSQNTNKHV EVNFIEKFTTERYFCPNTRCSITWFLSYSPCGECSRAITEFLSRYPHVTLFIYIARLYHHADPE NRQGLRDLISSGVTIQIMTEQESGYCWRNFVNYSPSNEAHWPRYPHLWVRLYVLELYCIILG LPPCLNILRRKQPQLTFFTIALQSCHYQRLPPHILWATGLK*

\section{YE2 (rAPOBEC1 W90Y, R132E) ${ }^{18}$}

MSSETGPVAVDPTLRRRIEPHEFEVFFDPRELRKETCLLYEINWGGRHSIWRHTSQNTNKHV EVNFIEKFTTERYFCPNTRCSITWFLSYSPCGECSRAITEFLSRYPHVTLFIYIARLYHHADPR NRQGLEDLISSGVTIQIMTEQESGYCWRNFVNYSPSNEAHWPRYPHLWVRLYVLELYCIILG LPPCLNILRRKQPQLTFFTIALQSCHYQRLPPHILWATGLK*

YEE (rAPOBEC1 W90Y, R126E, R132E) ${ }^{18}$

MSSETGPVAVDPTLRRRIEPHEFEVFFDPRELRKETCLLYEINWGGRHSIWRHTSQNTNKHV EVNFIEKFTTERYFCPNTRCSITWFLSYSPCGECSRAITEFLSRYPHVTLFIYIARLYHHADPE NRQGLEDLISSGVTIQIMTEQESGYCWRNFVNYSPSNEAHWPRYPHLWVRLYVLELYCIILG LPPCLNILRRKQPQLTFFTIALQSCHYQRLPPHILWATGLK*

\section{Anc689 19}

MSSETGPVAVDPTLRRRIEPHEFEVFFDPRELRKETCLLYEIKWGTSHKIWRHSSKNTTKHV EVNFIEKFTSERHFCPSTSCSITWFLSWSPCGECSKAITEFLSQHPNVTLVIYVARLYHHMDQ QNRQGLRDLVNSGVTIQIMTAPEYDYCWRNFVNYPPGKEAHWPRYPPLWMKLYALELHAGI LGLPPCLNILRRKQPQLTFFTIALQSCHYQRLPPHI*

\section{eA3A 20}

MEASPASGPRHLMDPHIFTSNFNNGIGRHKTYLCYEVERLDNGTSVKMDQHRGFLHGQAK NLLCGFYGRHAELRFLDLVPSLQLDPAQIYRVTWFISWSPCFSWGCAGEVRAFLQENTHVR LRIFAARIYDYDPLYKEALQMLRDAGAQVSIMTYDEFKHCWDTFVDHQGCPFQPWDGLDEH SQALSGRLRAILQNQGN*

\section{eA3A* $(T 31 A)^{8}$}

MEASPASGPRHLMDPHIFTSNFNNGIGRHKAYLCYEVERLDNGTSVKMDQHRGFLHGQAK NLLCGFYGRHAELRFLDLVPSLQLDPAQIYRVTWFISWSPCFSWGCAGEVRAFLQENTHVR LRIFAARIYDYDPLYKEALQMLRDAGAQVSIMTYDEFKHCWDTFVDHQGCPFQPWDGLDEH SQALSGRLRAILQNQGN* 
Glycosylase fusion domains

Udg $X^{21}$

MAGAQDFVPHTADLAELAAAAGECRGCGLYRDATQAVFGAGGRSARIMMIGEQPGDKEDL AGLPFVGPAGRLLDRALEAADIDRDALYVTNAVKHFKFTRAAGGKRRIHKTPSRTEVVACRP WLIAEMTSVEPDVVVLLGATAAKALLGNDFRVTQHRGEVLHVDDVPGDPALVATVHPSSLLR GPKEERESAFAGLVDDLRVAADVRP*

\section{UNG2}

MIGQKTLYSFFSPSPARKRHAPSPEPAVQGTGVAGVPEESGDAAAIPAKKAPAGQEEPGTP PSSPLSAEQLDRIQRNKAAALLRLAARNVPVGFGESWKKHLSGEFGKPYFIKLMGFVAEERK HYTVYPPPHQVFTWTQMCDIKDVKVVILGQDPYHGPNQAHGLCFSVQRPVPPPPSLENIYK ELSTDIEDFVHPGHGDLSGWAKQGVLLLNAVLTVRAHQANSHKERGWEQFTDAVVSWLNQ NSNGLVFLLWGSYAQKKGSAIDRKRHHVLQTAHPSPLSVYRGFFGCRHFSKTNELLQKSGK KPIDWKEL*

\section{SMUG1}

MPQAFLLGSIHEPAGALMEPQPCPGSLAESFLEEELRLNAELSQLQFSEPVGIIYNPVEYAW EPHRNYVTRYCQGPKEVLFLGMNPGPFGMAQTGVPFGEVSMVRDWLGIVGPVLTPPQEHP KRPVLGLECPQSEVSGARFWGFFRNLCGQPEVFFHHCFVHNLCPLLFLAPSGRNLTPAELP AKQREQLLGICDAALCRQVQLLGVRLVVGVGRLAEQRARRALAGLMPEVQVEGLLHPSPRN PQANKGWEAVAKERLNELGLLPLLLK*

\section{MBD4}

MGTTGLESLSLGDRGAAPTVTSSERLVPDPPNDLRKEDVAMELERVGEDEEQMMIKRSSE CNPLLQEPIASAQFGATAGTECRKSVPCGWERVVKQRLFGKTAGRFDVYFISPQGLKFRSK SSLANYLHKNGETSLKPEDFDFTVLSKRGIKSRYKDCSMAALTSHLQNQSNNSNWNLRTRS KCKKDVFMPPSSSSELQESRGLSNFTSTHLLLKEDEGVDDVNFRKVRKPKGKVTILKGIPIKK TKKGCRKSCSGFVQSDSKRESVCNKADAESEPVAQKSQLDRTVCISDAGACGETLSVTSEE NSLVKKKERSLSSGSNFCSEQKTSGIINKFCSAKDSEHNEKYEDTFLESEEIGTKVEVVERKE HLHTDILKRGSEMDNNCSPTRKDFTGEKIFQEDTIPRTQIERRKTSLYFSSKYNKEALSPPRR KAFKKWTPPRSPFNLVQETLFHDPWKLLIATIFLNRTSGKMAIPVLWKFLEKYPSAEVARTAD WRDVSELLKPLGLYDLRAKTIVKFSDEYLTKQWKYPIELHGIGKYGNDSYRIFCVNEWKQVH PEDHKLNKYHDWLWENHEKLSL*

\section{TDG}

MEAENAGSYSLQQAQAFYTFPFQQLMAEAPNMAVVNEQQMPEEVPAPAPAQEPVQEAPK GRKRKPRTTEPKQPVEPKKPVESKKSGKSAKSKEKQEKITDTFKVKRKVDRFNGVSEAELLT KTLPDILTFNLDIVIIGINPGLMAAYKGHHYPGPGNHFWKCLFMSGLSEVQLNHMDDHTLPGK YGIGFTNMVERTTPGSKDLSSKEFREGGRILVQKLQKYQPRIAVFNGKCIYEIFSKEVFGVKV KNLEFGLQPHKIPDTETLCYVMPSSSARCAQFPRAQDKVHYYIKLKDLRDQLKGIERNMDVQ EVQYTFDLQLAQEDAKKMAVKEEKYDPGYEAAYGGAYGENPCSSEPCGFSSNGLIESVELR GESAFSGIPNGQWMTQSFTDQIPSFSNHCGTQEQEEESHA*

CRISPRi screen hit fusion domains

\section{DDX1}

MAAFSEMGVMPEIAQAVEEMDWLLPTDIQAESIPLILGGGDVLMAAETGSGKTGAFSIPVIQI VYETLKDQQEGKKGKTTIKTGASVLNKWQMNPYDRGSAFAIGSDGLCCQSREVKEWHGCR ATKGLMKGKHYYEVSCHDQGLCRVGWSTMQASLDLGTDKFGFGFGGTGKKSHNKQFDNY GEEFTMHDTIGCYLDIDKGHVKFSKNGKDLGLAFEIPPHMKNQALFPACVLKNAELKFNFGE 
EEFKFPPKDGFVALSKAPDGYIVKSQHSGNAQVTQTKFLPNAPKALIVEPSRELAEQTLNNIK QFKKYIDNPKLRELLIIGGVAARDQLSVLENGVDIVVGTPGRLDDLVSTGKLNLSQVRFLVLD EADGLLSQGYSDFINRMHNQIPQVTSDGKRLQVIVCSATLHSFDVKKLSEKIMHFPTWVDLK GEDSVPDTVHHVVVPVNPKTDRLWERLGKSHIRTDDVHAKDNTRPGANSPEMWSEAIKILK GEYAVRAIKEHKMDQAIIFCRTKIDCDNLEQYFIQQGGGPDKKGHQFSCVCLHGDRKPHERK QNLERFKKGDVRFLICTDVAARGIDIHGVPYVINVTLPDEKQNYVHRIGRVGRAERMGLAISL VATEKEKVWYHVCSSRGKGCYNTRLKEDGGCTIWYNEMQLLSEIEEHLNCTISQVEPDIKVP VDEFDGKVTYGQKRAAGGGSYKGHVDILAPTVQELAALEKEAQTSFLHLGYLPNQLFRTF*

\section{EX01}

MGIQGLLQFIKEASEPIHVRKYKGQVVAVDTYCWLHKGAIACAEKLAKGEPTDRYVGFCMKF VNMLLSHGIKPILVFDGCTLPSKKEVERSRRERRQANLLKGKQLLREGKVSEARECFTRSINI THAMAHKVIKAARSQGVDCLVAPYEADAQLAYLNKAGIVQAIITEDSDLLAFGCKKVILKMDQ FGNGLEIDQARLGMCRQLGDVFTEEKFRYMCILSGCDYLSSLRGIGLAKACKVLRLANNPDI VKVIKKIGHYLKMNITVPEDYINGFIRANNTFLYQLVFDPIKRKLIPLNAYEDDVDPETLSYAGQ YVDDSIALQIALGNKDINTFEQIDDYNPDTAMPAHSRSHSWDDKTCQKSANVSSIWHRNYSP RPESGTVSDAPQLKENPSTVGVERVISTKGLNLPRKSSIVKRPRSAELSEDDLLSQYSLSFTK KTKKNSSEGNKSLSFSEVFVPDLVNGPTNKKSVSTPPRTRNKFATFLQRKNEESGAVVVPG TRSRFFCSSDSTDCVSNKVSIQPLDETAVTDKENNLHESEYGDQEGKRLVDTDVARNSSDDI PNNHIPGDHIPDKATVFTDEESYSFESSKFTRTISPPTLGTLRSCFSWSGGLGDFSRTPSPSP STALQQFRRKSDSPTSLPENNMSDVSQLKSEESSDDESHPLREEACSSQSQESGEFSLQS SNASKLSQCSSKDSDSEESDCNIKLLDSQSDQTSKLRLSHFSKKDTPLRNKVPGLYKSSSAD SLSTTKIKPLGPARASGLSKKPASIQKRKHHNAENKPGLQIKLNELWKNFGFKKDSEKLPPCK KPLSPVRDNIQLTPEAEEDIFNKPECGRVQRAIFQ*

\section{PCNA}

MFEARLVQGSILKKVLEALKDLINEACWDISSSGVNLQSMDSSHVSLVQLTLRSEGFDTYRC DRNLAMGVNLTSMSKILKCAGNEDIITLRAEDNADTLALVFEAPNQEKVSDYEMKLMDLDVE QLGIPEQEYSCVVKMPSGEFARICRDLSHIGDAVVISCAKDGVKFSASGELGNGNIKLSQTSN VDKEEEAVTIEMNEPVQLTFALRYLNFFTKATPLSSTVTLSMSADVPLVVEYKIADMGHLKYY LAPKIEDEEGS*

\section{POLD1}

MDGKRRPGPGPGVPPKRARGGLWDDDDAPRPSQFEEDLALMEEMEAEHRLQEQEEEELQ SVLEGVADGQVPPSAIDPRWLRPTPPALDPQTEPLIFQQLEIDHYVGPAQPVPGGPPPSRG SVPVLRAFGVTDEGFSVCCHIHGFAPYFYTPAPPGFGPEHMGDLQRELNLAISRDSRGGRE LTGPAVLAVELCSRESMFGYHGHGPSPFLRITVALPRLVAPARRLLEQGIRVAGLGTPSFAP YEANVDFEIRFMVDTDIVGCNWLELPAGKYALRLKEKATQCQLEADVLWSDVVSHPPEGPW QRIAPLRVLSFDIECAGRKGIFPEPERDPVIQICSLGLRWGEPEPFLRLALTLRPCAPILGAKV QSYEKEEDLLQAWSTFIRIMDPDVITGYNIQNFDLPYLISRAQTLKVQTFPFLGRVAGLCSNIR DSSFQSKQTGRRDTKVVSMVGRVQMDMLQVLLREYKLRSYTLNAVSFHFLGEQKEDVQHS IITDLQNGNDQTRRRLAVYCLKDAYLPLRLLERLMVLVNAVEMARVTGVPLSYLLSRGQQVK VVSQLLRQAMHEGLLMPVVKSEGGEDYTGATVIEPLKGYYDVPIATLDFSSLYPSIMMAHNL CYTTLLRPGTAQKLGLTEDQFIRTPTGDEFVKTSVRKGLLPQILENLLSARKRAKAELAKETD

PLRRQVLDGRQLALKVSANSVYGFTGAQVGKLPCLEISQSVTGFGRQMIEKTKQLVESKYTV ENGYSTSAKVVYGDTDSVMCRFGVSSVAEAMALGREAADWVSGHFPSPIRLEFEKVYFPYL LISKKRYAGLLFSSRPDAHDRMDCKGLEAVRRDNCPLVANLVTASLRRLLIDRDPEGAVAHA QDVISDLLCNRIDISQLVITKELTRAASDYAGKQAHVELAERMRKRDPGSAPSLGDRVPYVIIS AAKGVAAYMKSEDPLFVLEHSLPIDTQYYLEQQLAKPLLRIFEPILGEGRAEAVLLRGDHTRC 
KTVLTGKVGGLLAFAKRRNCCIGCRTVLSHQGAVCEFCQPRESELYQKEVSHLNALEERFS RLWTQCQRCQGSLHEDVICTSRDCPIFYMRKKVRKDLEDQEQLLRRFGPPGPEAW*

\section{POLD2}

MFSEQAAQRAHTLLSPPSANNATFARVPVATYTNSSQPFRLGERSFSRQYAHIYATRLIQMR PFLENRAQQHWGSGVGVKKLCELQPEEKCCVVGTLFKAMPLQPSILREVSEEHNLLPQPPR SKYIHPDDELVLEDELQRIKLKGTIDVSKLVTGTVLAVFGSVRDDGKFLVEDYCFADLAPQKP APPLDTDRFVLLVSGLGLGGGGGESLLGTQLLVDVVTGQLGDEGEQCSAAHVSRVILAGNL LSHSTQSRDSINKAKYLTKKTQAASVEAVKMLDEILLQLSASVPVDVMPGEFDPTNYTLPQQ PLHPCMFPLATAYSTLQLVTNPYQATIDGVRFLGTSGQNVSDIFRYSSMEDHLEILEWTLRVR HISPTAPDTLGCYPFYKTDPFIFPECPHVYFCGNTPSFGSKIIRGPEDQTVLLVTVPDFSATQT ACLVNLRSLACQPISFSGFGAEDDDLGGLGLGP*

\section{POLD3}

MADQLYLENIDEFVTDQNKIVTYKWLSYTLGVHVNQAKQMLYDYVERKRKENSGAQLHVTY LVSGSLIQNGHSCHKVAVVREDKLEAVKSKLAVTASIHVYSIQKAMLKDSGPLFNTDYDILKS NLQNCSKFSAIQCAAAVPRAPAESSSSSKKFEQSHLHMSSETQANNELTTNGHGPPASKQV SQQPKGIMGMFASKAAAKTQETNKETKTEAKEVTNASAAGNKAPGKGNMMSNFFGKAAMN KFKVNLDSEQAVKEEKIVEQPTVSVTEPKLATPAGLKKSSKKAEPVKVLQKEKKRGKRVALS DDETKETENMRKKRRRIKLPESDSSEDEVFPDSPGAYEAESPSPPPPPSPPLEPVPKTEPEP PSVKSSSGENKRKRKRVLKSKTYLDGEGCIVTEKVYESESCTDSEEELNMKTSSVHRPPAM TVKKEPREERKGPKKGTAALGKANRQVSITGFFQRK*

\section{POLH}

MATGQDRVVALVDMDCFFVQVEQRQNPHLRNKPCAVVQYKSWKGGGIIAVSYEARAFGVT RSMWADDAKKLCPDLLLAQVRESRGKANLTKYREASVEVMEIMSRFAVIERASIDEAYVDLT SAVQERLQKLQGQPISADLLPSTYIEGLPQGPTTAEETVQKEGMRKQGLFQWLDSLQIDNLT SPDLQLTVGAVIVEEMRAAIERETGFQCSAGISHNKVLAKLACGLNKPNRQTLVSHGSVPQL FSQMPIRKIRSLGGKLGASVIEILGIEYMGELTQFTESQLQSHFGEKNGSWLYAMCRGIEHDP VKPRQLPKTIGCSKNFPGKTALATREQVQWWLLQLAQELEERLTKDRNDNDRVATQLVVSI RVQGDKRLSSLRRCCALTRYDAHKMSHDAFTVIKNCNTSGIQTEWSPPLTMLFLCATKFSAS APSSSTDITSFLSSDPSSLPKVPVTSSEAKTQGSGPAVTATKKATTSLESFFQKAAERQKVK EASLSSLTAPTQAPMSNSPSKPSLPFQTSQSTGTEPFFKQKSLLLKQKQLNNSSVSSPQQN PWSNCKALPNSLPTEYPGCVPVCEGVSKLEESSKATPAEMDLAHNSQSMHASSASKSVLE VTQKATPNPSLLAAEDQVPCEKCGSLVPVWDMPEHMDYHFALELQKSFLQPHSSNPQVVS AVSHQGKRNPKSPLACTNKRPRPEGMQTLESFFKPLTH

\section{POLK}

MDSTKEKCDSYKDDLLLRMGLNDNKAGMEGLDKEKINKIIMEATKGSRFYGNELKKEKQVN QRIENMMQQKAQITSQQLRKAQLQVDRFAMELEQSRNLSNTIVHIDMDAFYAAVEMRDNPE LKDKPIAVGSMSMLSTSNYHARRFGVRAAMPGFIAKRLCPQLIIVPPNFDKYRAVSKEVKEIL ADYDPNFMAMSLDEAYLNITKHLEERQNWPEDKRRYFIKMGSSVENDNPGKEVNKLSEHER SISPLLFEESPSDVQPPGDPFQVNFEEQNNPQILQNSVVFGTSAQEVVKEIRFRIEQKTTLTA SAGIAPNTMLAKVCSDKNKPNGQYQILPNRQAVMDFIKDLPIRKVSGIGKVTEKMLKALGIITC TELYQQRALLSLLFSETSWHYFLHISLGLGSTHLTRDGERKSMSVERTFSEINKAEEQYSLC QELCSELAQDLQKERLKGRTVTIKLKNVNFEVKTRASTVSSVVSTAEEIFAIAKELLKTEIDAD FPHPLRLRLMGVRISSFPNEEDRKHQQRSIIGFLQAGNQALSATECTLEKTDKDKFVKPLEM SHKKSFFDKKRSERKWSHQDTFKCEAVNKQSFQTSQPFQVLKKKMNENLEISENSDDCQIL TCPVCFRAQGCISLEALNKHVDECLDGPSISENFKMFSCSHVSATKVNKKENVPASSLCEKQ DYEAHPKIKEISSVDCIALVDTIDNSSKAESIDALSNKHSKEECSSLPSKSFNIEHCHQNSSST 
VSLENEDVGSFRQEYRQPYLCEVKTGQALVCPVCNVEQKTSDLTLFNVHVDVCLNKSFIQEL RKDKFNPVNQPKESSRSTGSSSGVQKAVTRTKRPGLMTKYSTSKKIKPNNPKHTLDIFFK*

\section{RAD18}

MDSLAESRWPPGLAVMKTIDDLLRCGICFEYFNIAMIIPQCSHNYCSLCIRKFLSYKTQCPTC CVTVTEPDLKNNRILDELVKSLNFARNHLLQFALESPAKSPASSSSKNLAVKVYTPVASRQSL KQGSRLMDNFLIREMSGSTSELLIKENKSKFSPQKEASPAAKTKETRSVEEIAPDPSEAKRPE PPSTSTLKQVTKVDCPVCGVNIPESHINKHLDSCLSREEKKESLRSSVHKRKPLPKTVYNLLS DRDLKKKLKEHGLSIQGNKQQLIKRHQEFVHMYNAQCDALHPKSAAEIVREIENIEKTRMRLE ASKLNESVMVFTKDQTEKEIDEIHSKYRKKHKSEFQLLVDQARKGYKKIAGMSQKTVTITKED ESTEKLSSVCMGQEDNMTSVTNHFSQSKLDSPEELEPDREEDSSSCIDIQEVLSSSESDSC NSSSSDIIRDLLEEEEAWEASHKNDLQDTEISPRQNRRTRAAESAEIEPRNKRNRN*

\section{RBMX}

MVEADRPGKLFIGGLNTETNEKALEAVFGKYGRIVEVLLMKDRETNKSRGFAFVTFESPADA KDAARDMNGKSLDGKAIKVEQATKPSFESGRRGPPPPPRSRGPPRGLRGGRGGSGGTRG PPSRGGHMDDGGYSMNFNMSSSRGPLPVKRGPPPRSGGPPPKRSAPSGPVRSSSGMGG RAPVSRGRDSYGGPPRREPLPSRRDVYLSPRDDGYSTKDSYSSRDYPSSRDTRDYAPPPR DYTYRDYGHSSSRDDYPSRGYSDRDGYGRDRDYSDHPSGGSYRDSYESYGNSRSAPPTR GPPPSYGGSSRYDDYSSSRDGYGGSRDSYSSSRSDLYSSGRDRVGRQERGLPPSMERGY PPPRDSYSSSSRGAPRGGGRGGSRSDRGGGRSRY*

\section{REV1}

MRRGGWRKRAENDGWETWGGYMAAKVQKLEEQFRSDAAMQKDGTSSTIFSGVAIYVNGY TDPSAEELRKLMMLHGGQYHVYYSRSKTTHIIATNLPNAKIKELKGEKVIRPEWIVESIKAGRL LSYIPYQLYTKQSSVQKGLSFNPVCRPEDPLPGPSNIAKQLNNRVNHIVKKIETENEVKVNGM NSWNEEDENNDFSFVDLEQTSPGRKQNGIPHPRGSTAIFNGHTPSSNGALKTQDCLVPMV NSVASRLSPAFSQEEDKAEKSSTDFRDCTLQQLQQSTRNTDALRNPHRTNSFSLSPLHSNT KINGAHHSTVQGPSSTKSTSSVSTFSKAAPSVPSKPSDCNFISNFYSHSRLHHISMWKCELT EFVNTLQRQSNGIFPGREKLKKMKTGRSALVVTDTGDMSVLNSPRHQSCIMHVDMDCFFVS VGIRNRPDLKGKPVAVTSNRGTGRAPLRPGANPQLEWQYYQNKILKGKAADIPDSSLWENP DSAQANGIDSVLSRAEIASCSYEARQLGIKNGMFFGHAKQLCPNLQAVPYDFHAYKEVAQTL YETLASYTHNIEAVSCDEALVDITEILAETKLTPDEFANAVRMEIKDQTKCAASVGIGSNILLAR MATRKAKPDGQYHLKPEEVDDFIRGQLVTNLPGVGHSMESKLASLGIKTCGDLQYMTMAKL QKEFGPKTGQMLYRFCRGLDDRPVRTEKERKSVSAEINYGIRFTQPKEAEAFLLSLSEEIQR RLEATGMKGKRLTLKIMVRKPGAPVETAKFGGHGICDNIARTVTLDQATDNAKIIGKAMLNMF HTMKLNISDMRGVGIHVNQLVPTNLNPSTCPSRPSVQSSHFPSGSYSVRDVFQVQKAKKST EEEHKEVFRAAVDLEISSASRTCTFLPPFPAHLPTSPDTNKAESSGKWNGLHTPVSVQSRLN LSIEVPSPSQLDQSVLEALPPDLREQVEQVCAVQQAESHGDKKKEPVNGCNTGILPQPVGT VLLQIPEPQESNSDAGINLIALPAFSQVDPEVFAALPAELQRELKAAYDQRQRQGENSTHQQ SASASVPKNPLLHLKAAVKEKKRNKKKKTIGSPKRIQSPLNNKLLNSPAKTLPGACGSPQKLI DGFLKHEGPPAEKPLEELSASTSGVPGLSSLQSDPAGCVRPPAPNLAGAVEFNDVKTLLRE WITTISDPMEEDILQVVKYCTDLIEEKDLEKLDLVIKYMKRLMQQSVESVWNMAFDFILDNVQ VVLQQTYGSTLKVT*

\section{RFWD3}

MAHEAMEYDVQVQLNHAEQQPAPAGMASSQGGPALLQPVPADVVSSQGVPSILQPAPAEV ISSQATPPLLQPAPQLSVDLTEVEVLGEDTVENINPRTSEQHRQGSDGNHTIPASSLHSMTN FISGLQRLHGMLEFLRPSSSNHSVGPMRTRRRVSASRRARAGGSQRTDSARLRAPLDAYF QVSRTQPDLPATTYDSETRNPVSEELQVSSSSDSDSDSSAEYGGVVDQAEESGAVILEEQL 
AGVSAEQEVTCIDGGKTLPKQPSPQKSEPLLPSASMDEEEGDTCTICLEQWTNAGDHRLSA LRCGHLFGYRCISTWLKGQVRKCPQCNKKARHSDIVVLYARTLRALDTSEQERMKSSLLKE QMLRKQAELESAQCRLQLQVLTDKCTRLQRRVQDLQKLTSHQSQNLQQPRGSQAWVLSC SPSSQGQHKHKYHFQKTFTVSQAGNCRIMAYCDALSCLVISQPSPQASFLPGFGVKMLSTA NMKSSQYIPMHGKQIRGLAFSSYLRGLLLSASLDNTIKLTSLETNTVVQTYNAGRPVWSCCW CLDEANYIYAGLANGSILVYDVRNTSSHVQELVAQKARCPLVSLSYMPRAASAAFPYGGVLA GTLEDASFWEQKMDFSHWPHVLPLEPGGCIDFQTENSSRHCLVTYRPDKNHTTIRSVLMEM SYRLDDTGNPICSCQPVHTFFGGPTCKLLTKNAIFQSPENDGNILVCTGDEAANSALLWDAA SGSLLQDLQTDQPVLDICPFEVNRNSYLATLTEKMVHIYKWE*

\section{TIMELESS}

MDLHMMNCELLATCSALGYLEGDTYHKEPDCLESVKDLIRYLRHEDETRDVRQQLGAAQIL QSDLLPILTQHHQDKPLFDAVIRLMVNLTQPALLCFGNLPKEPSFRHHFLQVLTYLQAYKEAF ASEKAFGVLSETLYELLQLGWEERQEEDNLLIERILLLVRNILHVPADLDQEKKIDDDASAHD QLLWAIHLSGLDDLLLFLASSSAEEQWSLHVLEIVSLMFRDQNPEQLAGVGQGRLAQERSA DFAELEVLRQREMAEKKTRALQRGNRHSRFGGSYIVQGLKSIGERDLIFHKGLHNLRNYSSD LGKQPKKVPKRRQAARELSIQRRSALNVRLFLRDFCSEFLENCYNRLMGSVKDHLLREKAQ QHDETYYMWALAFFMAFNRAASFRPGLVSETLSVRTFHFIEQNLTNYYEMMLTDRKEAASW ARRMHLALKAYQELLATVNEMDISPDEAVRESSRIIKNNIFYVMEYRELFLALFRKFDERCQP RSFLRDLVETTHLFLKMLERFCRSRGNLVVQNKQKKRRKKKKKVLDQAIVSGNVPSSPEEV EAVWPALAEQLQCCAQNSELSMDSVVPFDAASEVPVEEQRAEAMVRIQDCLLAGQAPQAL TLLRSAREVWPEGDVFGSQDISPEEEIQLLKQILSAPLPRQQGPEERGAEEEEEEEEEEEEE LQVVQVSEKEFNFLDYLKRFACSTVVRAYVLLLRSYQQNSAHTNHCIVKMLHRLAHDLKMEA LLFQLSVFCLFNRLLSDPAAGAYKELVTFAKYILGKFFALAAVNQKAFVELLFWKNTAVVREM TEGYGSLDDRSSSRRAPTWSPEEEAHLRELYLANKDVEGQDVVEAILAHLNTVPRTRKQIIH HLVQMGLADSVKDFQRKGTHIVLWTGDQELELQRLFEEFRDSDDVLGHIMKNITAKRSRARI VDKLLALGLVAERRELYKKRQKKLASSILPNGAESLKDFCQEDLEEEENLPEEDSEEEEEGG SEAEQVQGSLVLSNENLGQSLHQEGFSIPLLWLQNCLIRAADDREEDGCSQAVPLVPLTEE NEEAMENEQFQQLLRKLGVRPPASGQETFWRIPAKLSPTQLRRAAASLSQPEEEQKLQPEL QPKVPGEQGSDEEHCKEHRAQALRALLLAHKKKAGLASPEEEDAVGKEPLKAAPKKRQLLD SDEEQEEDEGRNRAPELGAPGIQKKKRYQIEDDEDD*

\section{UBE2I}

MSGIALSRLAQERKAWRKDHPFGFVAVPTKNPDGTMNLMNWECAIPGKKGTPWEGGLFKL RMLFKDDYPSSPPKCKFEPPLFHPNVYPSGTVCLSILEEDKDWRPAITIKQILLGIQELLNEPN IQDPAQAEAYTIYCQNRVEYEKRVRAQAKKFAPS*

\section{UBE2T}

MQRASRLKRELHMLATEPPPGITCWQDKDQMDDLRAQILGGANTPYEKGVFKLEVIIPERYP FEPPQIRFLTPIYHPNIDSAGRICLDVLKLPPKGAWRPSLNIATVLTSIQLLMSEPNPDDPLMA DISSEFKYNKPAFLKNARQWTEKHARQKQKADEEEMLDNLPEAGDSRVHNSTQKRKASQL VGIEKKFHPDV*

\section{UNG}

MIGQKTLYSFFSPSPARKRHAPSPEPAVQGTGVAGVPEESGDAAAIPAKKAPAGQEEPGTP PSSPLSAEQLDRIQRNKAAALLRLAARNVPVGFGESWKKHLSGEFGKPYFIKLMGFVAEERK HYTVYPPPHQVFTWTQMCDIKDVKVVILGQDPYHGPNQAHGLCFSVQRPVPPPPSLENIYK ELSTDIEDFVHPGHGDLSGWAKQGVLLLNAVLTVRAHQANSHKERGWEQFTDAVVSWLNQ NSNGLVFLLWGSYAQKKGSAIDRKRHHVLQTAHPSPLSVYRGFFGCRHFSKTNELLQKSGK KPIDWKEL* 
Cas9 effector domains (nickases $=\mathrm{D} 10 \mathrm{~A} ;$ dead $=\mathrm{D} 10 \mathrm{~A}, \mathrm{H} 840 \mathrm{~A})$ SpCas922-26

MDKKYSIGLDIGTNSVGWAVITDEYKVPSKKFKVLGNTDRHSIKKNLIGALLFDSGETAEATRL KRTARRRYTRRKNRICYLQEIFSNEMAKVDDSFFHRLEESFLVEEDKKHERHPIFGNIVDEVA YHEKYPTIYHLRKKLVDSTDKADLRLIYLALAHMIKFRGHFLIEGDLNPDNSDVDKLFIQLVQT YNQLFEENPINASGVDAKAILSARLSKSRRLENLIAQLPGEKKNGLFGNLIALSLGLTPNFKSN FDLAEDAKLQLSKDTYDDDLDNLLAQIGDQYADLFLAAKNLSDAILLSDILRVNTEITKAPLSAS MIKRYDEHHQDLTLLKALVRQQLPEKYKEIFFDQSKNGYAGYIDGGASQEEFYKFIKPILEKM DGTEELLVKLNREDLLRKQRTFDNGSIPHQIHLGELHAILRRQEDFYPFLKDNREKIEKILTFRI PYYVGPLARGNSRFAWMTRKSEETITPWNFEEVVDKGASAQSFIERMTNFDKNLPNEKVLP KHSLLYEYFTVYNELTKVKYVTEGMRKPAFLSGEQKKAIVDLLFKTNRKVTVKQLKEDYFKKI ECFDSVEISGVEDRFNASLGTYHDLLKIIKDKDFLDNEENEDILEDIVLTLTLFEDREMIEERLK TYAHLFDDKVMKQLKRRRYTGWGRLSRKLINGIRDKQSGKTILDFLKSDGFANRNFMQLIHD DSLTFKEDIQKAQVSGQGDSLHEHIANLAGSPAIKKGILQTVKVVDELVKVMGRHKPENIVIE MARENQTTQKGQKNSRERMKRIEEGIKELGSQILKEHPVENTQLQNEKLYLYYLQNGRDMY VDQELDINRLSDYDVDHIVPQSFLKDDSIDNKVLTRSDKNRGKSDNVPSEEVVKKMKNYWR QLLNAKLITQRKFDNLTKAERGGLSELDKAGFIKRQLVETRQITKHVAQILDSRMNTKYDEND KLIREVKVITLKSKLVSDFRKDFQFYKVREINNYHHAHDAYLNAVVGTALIKKYPKLESEFVYG DYKVYDVRKMIAKSEQEIGKATAKYFFYSNIMNFFKTEITLANGEIRKRPLIETNGETGEIVWD KGRDFATVRKVLSMPQVNIVKKTEVQTGGFSKESILPKRNSDKLIARKKDWDPKKYGGFDSP TVAYSVLVVAKVEKGKSKKLKSVKELLGITIMERSSFEKNPIDFLEAKGYKEVKKDLIIKLPKYS LFELENGRKRMLASAGELQKGNELALPSKYVNFLYLASHYEKLKGSPEDNEQKQLFVEQHK HYLDEIIEQISEFSKRVILADANLDKVLSAYNKHRDKPIREQAENIIHLFTLTNLGAPAAFKYFDT TIDRKRYTSTKEVLDATLIHQSITGLYETRIDLSQLGGD*

HF-SpCas9 (N497A, R661A, Q695A, Q926A) ${ }^{27}$

MDKKYSIGLDIGTNSVGWAVITDEYKVPSKKFKVLGNTDRHSIKKNLIGALLFDSGETAEATRL KRTARRRYTRRKNRICYLQEIFSNEMAKVDDSFFHRLEESFLVEEDKKHERHPIFGNIVDEVA YHEKYPTIYHLRKKLVDSTDKADLRLIYLALAHMIKFRGHFLIEGDLNPDNSDVDKLFIQLVQT YNQLFEENPINASGVDAKAILSARLSKSRRLENLIAQLPGEKKNGLFGNLIALSLGLTPNFKSN FDLAEDAKLQLSKDTYDDDLDNLLAQIGDQYADLFLAAKNLSDAILLSDILRVNTEITKAPLSAS MIKRYDEHHQDLTLLKALVRQQLPEKYKEIFFDQSKNGYAGYIDGGASQEEFYKFIKPILEKM DGTEELLVKLNREDLLRKQRTFDNGSIPHQIHLGELHAILRRQEDFYPFLKDNREKIEKILTFRI PYYVGPLARGNSRFAWMTRKSEETITPWNFEEVVDKGASAQSFIERMTAFDKNLPNEKVLP KHSLLYEYFTVYNELTKVKYVTEGMRKPAFLSGEQKKAIVDLLFKTNRKVTVKQLKEDYFKKI ECFDSVEISGVEDRFNASLGTYHDLLKIIKDKDFLDNEENEDILEDIVLTLTLFEDREMIEERLK TYAHLFDDKVMKQLKRRRYTGWGALSRKLINGIRDKQSGKTILDFLKSDGFANRNFMALIHD DSLTFKEDIQKAQVSGQGDSLHEHIANLAGSPAIKKGILQTVKVVDELVKVMGRHKPENIVIE MARENQTTQKGQKNSRERMKRIEEGIKELGSQILKEHPVENTQLQNEKLYLYYLQNGRDMY VDQELDINRLSDYDVDHIVPQSFLKDDSIDNKVLTRSDKNRGKSDNVPSEEVVKKMKNYWR QLLNAKLITQRKFDNLTKAERGGLSELDKAGFIKRQLVETRAITKHVAQILDSRMNTKYDEND KLIREVKVITLKSKLVSDFRKDFQFYKVREINNYHHAHDAYLNAVVGTALIKKYPKLESEFVYG DYKVYDVRKMIAKSEQEIGKATAKYFFYSNIMNFFKTEITLANGEIRKRPLIETNGETGEIVWD KGRDFATVRKVLSMPQVNIVKKTEVQTGGFSKESILPKRNSDKLIARKKDWDPKKYGGFDSP TVAYSVLVVAKVEKGKSKKLKSVKELLGITIMERSSFEKNPIDFLEAKGYKEVKKDLIIKLPKYS LFELENGRKRMLASAGELQKGNELALPSKYVNFLYLASHYEKLKGSPEDNEQKQLFVEQHK HYLDEIIEQISEFSKRVILADANLDKVLSAYNKHRDKPIREQAENIIHLFTLTNLGAPAAFKYFDT TIDRKRYTSTKEVLDATLIHQSITGLYETRIDLSQLGGD* 
e-SpCas9 (K848A, K1003A, R1060A) ${ }^{28}$

MDKKYSIGLDIGTNSVGWAVITDEYKVPSKKFKVLGNTDRHSIKKNLIGALLFDSGETAEATRL KRTARRRYTRRKNRICYLQEIFSNEMAKVDDSFFHRLEESFLVEEDKKHERHPIFGNIVDEVA YHEKYPTIYHLRKKLVDSTDKADLRLIYLALAHMIKFRGHFLIEGDLNPDNSDVDKLFIQLVQT YNQLFEENPINASGVDAKAILSARLSKSRRLENLIAQLPGEKKNGLFGNLIALSLGLTPNFKSN FDLAEDAKLQLSKDTYDDDLDNLLAQIGDQYADLFLAAKNLSDAILLSDILRVNTEITKAPLSAS MIKRYDEHHQDLTLLKALVRQQLPEKYKEIFFDQSKNGYAGYIDGGASQEEFYKFIKPILEKM DGTEELLVKLNREDLLRKQRTFDNGSIPHQIHLGELHAILRRQEDFYPFLKDNREKIEKILTFRI PYYVGPLARGNSRFAWMTRKSEETITPWNFEEVVDKGASAQSFIERMTNFDKNLPNEKVLP KHSLLYEYFTVYNELTKVKYVTEGMRKPAFLSGEQKKAIVDLLFKTNRKVTVKQLKEDYFKKI ECFDSVEISGVEDRFNASLGTYHDLLKIIKDKDFLDNEENEDILEDIVLTLTLFEDREMIEERLK TYAHLFDDKVMKQLKRRRYTGWGRLSRKLINGIRDKQSGKTILDFLKSDGFANRNFMQLIHD DSLTFKEDIQKAQVSGQGDSLHEHIANLAGSPAIKKGILQTVKVVDELVKVMGRHKPENIVIE MARENQTTQKGQKNSRERMKRIEEGIKELGSQILKEHPVENTQLQNEKLYLYYLQNGRDMY VDQELDINRLSDYDVDHIVPQSFLADDSIDNKVLTRSDKNRGKSDNVPSEEVVKKMKNYWR QLLNAKLITQRKFDNLTKAERGGLSELDKAGFIKRQLVETRQITKHVAQILDSRMNTKYDEND KLIREVKVITLKSKLVSDFRKDFQFYKVREINNYHHAHDAYLNAVVGTALIKKYPALESEFVYG DYKVYDVRKMIAKSEQEIGKATAKYFFYSNIMNFFKTEITLANGEIRKAPLIETNGETGEIVWD KGRDFATVRKVLSMPQVNIVKKTEVQTGGFSKESILPKRNSDKLIARKKDWDPKKYGGFDSP TVAYSVLVVAKVEKGKSKKLKSVKELLGITIMERSSFEKNPIDFLEAKGYKEVKKDLIIKLPKYS LFELENGRKRMLASAGELQKGNELALPSKYVNFLYLASHYEKLKGSPEDNEQKQLFVEQHK HYLDEIIEQISEFSKRVILADANLDKVLSAYNKHRDKPIREQAENIIHLFTLTNLGAPAAFKYFDT TIDRKRYTSTKEVLDATLIHQSITGLYETRIDLSQLGGD*

Hypa-Cas9 (N692A, M694A, Q695A, D1135E) ${ }^{29}$

MDKKYSIGLDIGTNSVGWAVITDEYKVPSKKFKVLGNTDRHSIKKNLIGALLFDSGETAEATRL KRTARRRYTRRKNRICYLQEIFSNEMAKVDDSFFHRLEESFLVEEDKKHERHPIFGNIVDEVA YHEKYPTIYHLRKKLVDSTDKADLRLIYLALAHMIKFRGHFLIEGDLNPDNSDVDKLFIQLVQT YNQLFEENPINASGVDAKAILSARLSKSRRLENLIAQLPGEKKNGLFGNLIALSLGLTPNFKSN FDLAEDAKLQLSKDTYDDDLDNLLAQIGDQYADLFLAAKNLSDAILLSDILRVNTEITKAPLSAS MIKRYDEHHQDLTLLKALVRQQLPEKYKEIFFDQSKNGYAGYIDGGASQEEFYKFIKPILEKM DGTEELLVKLNREDLLRKQRTFDNGSIPHQIHLGELHAILRRQEDFYPFLKDNREKIEKILTFRI PYYVGPLARGNSRFAWMTRKSEETITPWNFEEVVDKGASAQSFIERMTNFDKNLPNEKVLP KHSLLYEYFTVYNELTKVKYVTEGMRKPAFLSGEQKKAIVDLLFKTNRKVTVKQLKEDYFKKI ECFDSVEISGVEDRFNASLGTYHDLLKIIKDKDFLDNEENEDILEDIVLTLTLFEDREMIEERLK TYAHLFDDKVMKQLKRRRYTGWGRLSRKLINGIRDKQSGKTILDFLKSDGFANRAFAALIHD DSLTFKEDIQKAQVSGQGDSLHEHIANLAGSPAIKKGILQTVKVVDELVKVMGRHKPENIVIE MARENQTTQKGQKNSRERMKRIEEGIKELGSQILKEHPVENTQLQNEKLYLYYLQNGRDMY VDQELDINRLSDYDVDHIVPQSFLKDDSIDNKVLTRSDKNRGKSDNVPSEEVVKKMKNYWR QLLNAKLITQRKFDNLTKAERGGLSELDKAGFIKRQLVETRQITKHVAQILDSRMNTKYDEND KLIREVKVITLKSKLVSDFRKDFQFYKVREINNYHHAHDAYLNAVVGTALIKKYPKLESEFVYG DYKVYDVRKMIAKSEQEIGKATAKYFFYSNIMNFFKTEITLANGEIRKRPLIETNGETGEIVWD KGRDFATVRKVLSMPQVNIVKKTEVQTGGFSKESILPKRNSDKLIARKKDWDPKKYGGFESP TVAYSVLVVAKVEKGKSKKLKSVKELLGITIMERSSFEKNPIDFLEAKGYKEVKKDLIIKLPKYS LFELENGRKRMLASAGELQKGNELALPSKYVNFLYLASHYEKLKGSPEDNEQKQLFVEQHK HYLDEIIEQISEFSKRVILADANLDKVLSAYNKHRDKPIREQAENIIHLFTLTNLGAPAAFKYFDT TIDRKRYTSTKEVLDATLIHQSITGLYETRIDLSQLGGD*

e-HF-SpCas9 (K848A, K1003A, R1060A/N497A, R661A, Q695A, Q926A) 
MDKKYSIGLDIGTNSVGWAVITDEYKVPSKKFKVLGNTDRHSIKKNLIGALLFDSGETAEATRL KRTARRRYTRRKNRICYLQEIFSNEMAKVDDSFFHRLEESFLVEEDKKHERHPIFGNIVDEVA YHEKYPTIYHLRKKLVDSTDKADLRLIYLALAHMIKFRGHFLIEGDLNPDNSDVDKLFIQLVQT YNQLFEENPINASGVDAKAILSARLSKSRRLENLIAQLPGEKKNGLFGNLIALSLGLTPNFKSN FDLAEDAKLQLSKDTYDDDLDNLLAQIGDQYADLFLAAKNLSDAILLSDILRVNTEITKAPLSAS MIKRYDEHHQDLTLLKALVRQQLPEKYKEIFFDQSKNGYAGYIDGGASQEEFYKFIKPILEKM DGTEELLVKLNREDLLRKQRTFDNGSIPHQIHLGELHAILRRQEDFYPFLKDNREKIEKILTFRI PYYVGPLARGNSRFAWMTRKSEETITPWNFEEVVDKGASAQSFIERMTAFDKNLPNEKVLP KHSLLYEYFTVYNELTKVKYVTEGMRKPAFLSGEQKKAIVDLLFKTNRKVTVKQLKEDYFKKI ECFDSVEISGVEDRFNASLGTYHDLLKIIKDKDFLDNEENEDILEDIVLTLTLFEDREMIEERLK TYAHLFDDKVMKQLKRRRYTGWGALSRKLINGIRDKQSGKTILDFLKSDGFANRNFMALIHD DSLTFKEDIQKAQVSGQGDSLHEHIANLAGSPAIKKGILQTVKVVDELVKVMGRHKPENIVIE MARENQTTQKGQKNSRERMKRIEEGIKELGSQILKEHPVENTQLQNEKLYLYYLQNGRDMY VDQELDINRLSDYDVDHIVPQSFLADDSIDNKVLTRSDKNRGKSDNVPSEEVVKKMKNYWR QLLNAKLITQRKFDNLTKAERGGLSELDKAGFIKRQLVETRAITKHVAQILDSRMNTKYDEND KLIREVKVITLKSKLVSDFRKDFQFYKVREINNYHHAHDAYLNAVVGTALIKKYPALESEFVYG DYKVYDVRKMIAKSEQEIGKATAKYFFYSNIMNFFKTEITLANGEIRKAPLIETNGETGEIVWD KGRDFATVRKVLSMPQVNIVKKTEVQTGGFSKESILPKRNSDKLIARKKDWDPKKYGGFDSP TVAYSVLVVAKVEKGKSKKLKSVKELLGITIMERSSFEKNPIDFLEAKGYKEVKKDLIIKLPKYS LFELENGRKRMLASAGELQKGNELALPSKYVNFLYLASHYEKLKGSPEDNEQKQLFVEQHK HYLDEIIEQISEFSKRVILADANLDKVLSAYNKHRDKPIREQAENIIHLFTLTNLGAPAAFKYFDT TIDRKRYTSTKEVLDATLIHQSITGLYETRIDLSQLGGD*

\section{e-Hypa-SpCas9 (K848A, K1003A, R1060A/N692A, M694A, Q695A, D1135E)}

MDKKYSIGLDIGTNSVGWAVITDEYKVPSKKFKVLGNTDRHSIKKNLIGALLFDSGETAEATRL KRTARRRYTRRKNRICYLQEIFSNEMAKVDDSFFHRLEESFLVEEDKKHERHPIFGNIVDEVA YHEKYPTIYHLRKKLVDSTDKADLRLIYLALAHMIKFRGHFLIEGDLNPDNSDVDKLFIQLVQT YNQLFEENPINASGVDAKAILSARLSKSRRLENLIAQLPGEKKNGLFGNLIALSLGLTPNFKSN FDLAEDAKLQLSKDTYDDDLDNLLAQIGDQYADLFLAAKNLSDAILLSDILRVNTEITKAPLSAS MIKRYDEHHQDLTLLKALVRQQLPEKYKEIFFDQSKNGYAGYIDGGASQEEFYKFIKPILEKM DGTEELLVKLNREDLLRKQRTFDNGSIPHQIHLGELHAILRRQEDFYPFLKDNREKIEKILTFRI PYYVGPLARGNSRFAWMTRKSEETITPWNFEEVVDKGASAQSFIERMTNFDKNLPNEKVLP KHSLLYEYFTVYNELTKVKYVTEGMRKPAFLSGEQKKAIVDLLFKTNRKVTVKQLKEDYFKKI ECFDSVEISGVEDRFNASLGTYHDLLKIIKDKDFLDNEENEDILEDIVLTLTLFEDREMIEERLK TYAHLFDDKVMKQLKRRRYTGWGRLSRKLINGIRDKQSGKTILDFLKSDGFANRAFAALIHD DSLTFKEDIQKAQVSGQGDSLHEHIANLAGSPAIKKGILQTVKVVDELVKVMGRHKPENIVIE MARENQTTQKGQKNSRERMKRIEEGIKELGSQILKEHPVENTQLQNEKLYLYYLQNGRDMY VDQELDINRLSDYDVDHIVPQSFLADDSIDNKVLTRSDKNRGKSDNVPSEEVVKKMKNYWR QLLNAKLITQRKFDNLTKAERGGLSELDKAGFIKRQLVETRQITKHVAQILDSRMNTKYDEND KLIREVKVITLKSKLVSDFRKDFQFYKVREINNYHHAHDAYLNAVVGTALIKKYPALESEFVYG DYKVYDVRKMIAKSEQEIGKATAKYFFYSNIMNFFKTEITLANGEIRKAPLIETNGETGEIVWD KGRDFATVRKVLSMPQVNIVKKTEVQTGGFSKESILPKRNSDKLIARKKDWDPKKYGGFESP TVAYSVLVVAKVEKGKSKKLKSVKELLGITIMERSSFEKNPIDFLEAKGYKEVKKDLIIKLPKYS LFELENGRKRMLASAGELQKGNELALPSKYVNFLYLASHYEKLKGSPEDNEQKQLFVEQHK HYLDEIIEQISEFSKRVILADANLDKVLSAYNKHRDKPIREQAENIIHLFTLTNLGAPAAFKYFDT TIDRKRYTSTKEVLDATLIHQSITGLYETRIDLSQLGGD*

HF-Hypa-SpCas9 (N497A, R661A, Q695A, Q926A//N692A, M694A, Q695A, D1135E) MDKKYSIGLDIGTNSVGWAVITDEYKVPSKKFKVLGNTDRHSIKKNLIGALLFDSGETAEATRL KRTARRRYTRRKNRICYLQEIFSNEMAKVDDSFFHRLEESFLVEEDKKHERHPIFGNIVDEVA 
YHEKYPTIYHLRKKLVDSTDKADLRLIYLALAHMIKFRGHFLIEGDLNPDNSDVDKLFIQLVQT YNQLFEENPINASGVDAKAILSARLSKSRRLENLIAQLPGEKKNGLFGNLIALSLGLTPNFKSN FDLAEDAKLQLSKDTYDDDLDNLLAQIGDQYADLFLAAKNLSDAILLSDILRVNTEITKAPLSAS MIKRYDEHHQDLTLLKALVRQQLPEKYKEIFFDQSKNGYAGYIDGGASQEEFYKFIKPILEKM DGTEELLVKLNREDLLRKQRTFDNGSIPHQIHLGELHAILRRQEDFYPFLKDNREKIEKILTFRI PYYVGPLARGNSRFAWMTRKSEETITPWNFEEVVDKGASAQSFIERMTAFDKNLPNEKVLP KHSLLYEYFTVYNELTKVKYVTEGMRKPAFLSGEQKKAIVDLLFKTNRKVTVKQLKEDYFKKI ECFDSVEISGVEDRFNASLGTYHDLLKIIKDKDFLDNEENEDILEDIVLTLTLFEDREMIEERLK TYAHLFDDKVMKQLKRRRYTGWGRLSRKLINGIRDKQSGKTILDFLKSDGFANRAFAALIHD DSLTFKEDIQKAQVSGQGDSLHEHIANLAGSPAIKKGILQTVKVVDELVKVMGRHKPENIVIE MARENQTTQKGQKNSRERMKRIEEGIKELGSQILKEHPVENTQLQNEKLYLYYLQNGRDMY VDQELDINRLSDYDVDHIVPQSFLKDDSIDNKVLTRSDKNRGKSDNVPSEEVVKKMKNYWR QLLNAKLITQRKFDNLTKAERGGLSELDKAGFIKRQLVETRAITKHVAQILDSRMNTKYDEND KLIREVKVITLKSKLVSDFRKDFQFYKVREINNYHHAHDAYLNAVVGTALIKKYPKLESEFVYG DYKVYDVRKMIAKSEQEIGKATAKYFFYSNIMNFFKTEITLANGEIRKRPLIETNGETGEIVWD KGRDFATVRKVLSMPQVNIVKKTEVQTGGFSKESILPKRNSDKLIARKKDWDPKKYGGFESP TVAYSVLVVAKVEKGKSKKLKSVKELLGITIMERSSFEKNPIDFLEAKGYKEVKKDLIIKLPKYS LFELENGRKRMLASAGELQKGNELALPSKYVNFLYLASHYEKLKGSPEDNEQKQLFVEQHK HYLDEIIEQISEFSKRVILADANLDKVLSAYNKHRDKPIREQAENIIHLFTLTNLGAPAAFKYFDT TIDRKRYTSTKEVLDATLIHQSITGLYETRIDLSQLGGD*

\section{e-HF-Hypa-SpCas9 (K848A, K1003A, R1060A// I/N497A, R661A, Q695A, Q926A//N692A, M694A, Q695A, D1135E)}

MDKKYSIGLDIGTNSVGWAVITDEYKVPSKKFKVLGNTDRHSIKKNLIGALLFDSGETAEATRL KRTARRRYTRRKNRICYLQEIFSNEMAKVDDSFFHRLEESFLVEEDKKHERHPIFGNIVDEVA YHEKYPTIYHLRKKLVDSTDKADLRLIYLALAHMIKFRGHFLIEGDLNPDNSDVDKLFIQLVQT YNQLFEENPINASGVDAKAILSARLSKSRRLENLIAQLPGEKKNGLFGNLIALSLGLTPNFKSN FDLAEDAKLQLSKDTYDDDLDNLLAQIGDQYADLFLAAKNLSDAILLSDILRVNTEITKAPLSAS MIKRYDEHHQDLTLLKALVRQQLPEKYKEIFFDQSKNGYAGYIDGGASQEEFYKFIKPILEKM DGTEELLVKLNREDLLRKQRTFDNGSIPHQIHLGELHAILRRQEDFYPFLKDNREKIEKILTFRI PYYVGPLARGNSRFAWMTRKSEETITPWNFEEVVDKGASAQSFIERMTAFDKNLPNEKVLP KHSLLYEYFTVYNELTKVKYVTEGMRKPAFLSGEQKKAIVDLLFKTNRKVTVKQLKEDYFKKI ECFDSVEISGVEDRFNASLGTYHDLLKIIKDKDFLDNEENEDILEDIVLTLTLFEDREMIEERLK TYAHLFDDKVMKQLKRRRYTGWGRLSRKLINGIRDKQSGKTILDFLKSDGFANRAFAALIHD DSLTFKEDIQKAQVSGQGDSLHEHIANLAGSPAIKKGILQTVKVVDELVKVMGRHKPENIVIE MARENQTTQKGQKNSRERMKRIEEGIKELGSQILKEHPVENTQLQNEKLYLYYLQNGRDMY VDQELDINRLSDYDVDHIVPQSFLADDSIDNKVLTRSDKNRGKSDNVPSEEVVKKMKNYWR QLLNAKLITQRKFDNLTKAERGGLSELDKAGFIKRQLVETRAITKHVAQILDSRMNTKYDEND KLIREVKVITLKSKLVSDFRKDFQFYKVREINNYHHAHDAYLNAVVGTALIKKYPALESEFVYG DYKVYDVRKMIAKSEQEIGKATAKYFFYSNIMNFFKTEITLANGEIRKAPLIETNGETGEIVWD KGRDFATVRKVLSMPQVNIVKKTEVQTGGFSKESILPKRNSDKLIARKKDWDPKKYGGFESP TVAYSVLVVAKVEKGKSKKLKSVKELLGITIMERSSFEKNPIDFLEAKGYKEVKKDLIIKLPKYS LFELENGRKRMLASAGELQKGNELALPSKYVNFLYLASHYEKLKGSPEDNEQKQLFVEQHK HYLDEIIEQISEFSKRVILADANLDKVLSAYNKHRDKPIREQAENIIHLFTLTNLGAPAAFKYFDT TIDRKRYTSTKEVLDATLIHQSITGLYETRIDLSQLGGD*

\section{Sniper-Cas9 (F539S, M763I, K890N) ${ }^{30}$}

MDKKYSIGLDIGTNSVGWAVITDEYKVPSKKFKVLGNTDRHSIKKNLIGALLFDSGETAEATRL KRTARRRYTRRKNRICYLQEIFSNEMAKVDDSFFHRLEESFLVEEDKKHERHPIFGNIVDEVA YHEKYPTIYHLRKKLVDSTDKADLRLIYLALAHMIKFRGHFLIEGDLNPDNSDVDKLFIQLVQT 
YNQLFEENPINASGVDAKAILSARLSKSRRLENLIAQLPGEKKNGLFGNLIALSLGLTPNFKSN FDLAEDAKLQLSKDTYDDDLDNLLAQIGDQYADLFLAAKNLSDAILLSDILRVNTEITKAPLSAS MIKRYDEHHQDLTLLKALVRQQLPEKYKEIFFDQSKNGYAGYIDGGASQEEFYKFIKPILEKM DGTEELLVKLNREDLLRKQRTFDNGSIPHQIHLGELHAILRRQEDFYPFLKDNREKIEKILTFRI PYYVGPLARGNSRFAWMTRKSEETITPWNFEEVVDKGASAQSFIERMTNFDKNLPNEKVLP KHSLLYEYFTVYNELTKVKYVTEGMRKPASLSGEQKKAIVDLLFKTNRKVTVKQLKEDYFKKI ECFDSVEISGVEDRFNASLGTYHDLLKIIKDKDFLDNEENEDILEDIVLTLTLFEDREMIEERLK TYAHLFDDKVMKQLKRRRYTGWGRLSRKLINGIRDKQSGKTILDFLKSDGFANRNFMQLIHD DSLTFKEDIQKAQVSGQGDSLHEHIANLAGSPAIKKGILQTVKVVDELVKVMGRHKPENIVIEI ARENQTTQKGQKNSRERMKRIEEGIKELGSQILKEHPVENTQLQNEKLYLYYLQNGRDMYV DQELDINRLSDYDVDHIVPQSFLKDDSIDNKVLTRSDKNRGKSDNVPSEEVVKKMKNYWRQ LLNANLITQRKFDNLTKAERGGLSELDKAGFIKRQLVETRQITKHVAQILDSRMNTKYDENDK LIREVKVITLKSKLVSDFRKDFQFYKVREINNYHHAHDAYLNAVVGTALIKKYPKLESEFVYGD YKVYDVRKMIAKSEQEIGKATAKYFFYSNIMNFFKTEITLANGEIRKRPLIETNGETGEIVWDK GRDFATVRKVLSMPQVNIVKKTEVQTGGFSKESILPKRNSDKLIARKKDWDPKKYGGFDSPT VAYSVLVVAKVEKGKSKKLKSVKELLGITIMERSSFEKNPIDFLEAKGYKEVKKDLIIKLPKYSL FELENGRKRMLASAGELQKGNELALPSKYVNFLYLASHYEKLKGSPEDNEQKQLFVEQHKH YLDEIIEQISEFSKRVILADANLDKVLSAYNKHRDKPIREQAENIIHLFTLTNLGAPAAFKYFDTT IDRKRYTSTKEVLDATLIHQSITGLYETRIDLSQLGGD*

Cas9-NG (L111R, D1135V, G1218R, E1219F, A1322R, R1335V, T1337R) ${ }^{1}$

MDKKYSIGLDIGTNSVGWAVITDEYKVPSKKFKVLGNTDRHSIKKNLIGALLFDSGETAEATRL KRTARRRYTRRKNRICYLQEIFSNEMAKVDDSFFHRLEESFLVEEDKKHERHPIFGNIVDEVA YHEKYPTIYHLRKKLVDSTDKADLRLIYLALAHMIKFRGHFLIEGDLNPDNSDVDKLFIQLVQT YNQLFEENPINASGVDAKAILSARLSKSRRLENLIAQLPGEKKNGLFGNLIALSLGLTPNFKSN FDLAEDAKLQLSKDTYDDDLDNLLAQIGDQYADLFLAAKNLSDAILLSDILRVNTEITKAPLSAS MIKRYDEHHQDLTLLKALVRQQLPEKYKEIFFDQSKNGYAGYIDGGASQEEFYKFIKPILEKM DGTEELLVKLNREDLLRKQRTFDNGSIPHQIHLGELHAILRRQEDFYPFLKDNREKIEKILTFRI PYYVGPLARGNSRFAWMTRKSEETITPWNFEEVVDKGASAQSFIERMTNFDKNLPNEKVLP KHSLLYEYFTVYNELTKVKYVTEGMRKPAFLSGEQKKAIVDLLFKTNRKVTVKQLKEDYFKKI ECFDSVEISGVEDRFNASLGTYHDLLKIIKDKDFLDNEENEDILEDIVLTLTLFEDREMIEERLK TYAHLFDDKVMKQLKRRRYTGWGRLSRKLINGIRDKQSGKTILDFLKSDGFANRNFMQLIHD DSLTFKEDIQKAQVSGQGDSLHEHIANLAGSPAIKKGILQTVKVVDELVKVMGRHKPENIVIE MARENQTTQKGQKNSRERMKRIEEGIKELGSQILKEHPVENTQLQNEKLYLYYLQNGRDMY VDQELDINRLSDYDVDHIVPQSFLKDDSIDNKVLTRSDKNRGKSDNVPSEEVVKKMKNYWR QLLNAKLITQRKFDNLTKAERGGLSELDKAGFIKRQLVETRQITKHVAQILDSRMNTKYDEND KLIREVKVITLKSKLVSDFRKDFQFYKVREINNYHHAHDAYLNAVVGTALIKKYPKLESEFVYG DYKVYDVRKMIAKSEQEIGKATAKYFFYSNIMNFFKTEITLANGEIRKRPLIETNGETGEIVWD KGRDFATVRKVLSMPQVNIVKKTEVQTGGFSKESIRPKRNSDKLIARKKDWDPKKYGGFVS PTVAYSVLVVAKVEKGKSKKLKSVKELLGITIMERSSFEKNPIDFLEAKGYKEVKKDLIIKLPKY SLFELENGRKRMLASARFLQKGNELALPSKYVNFLYLASHYEKLKGSPEDNEQKQLFVEQH KHYLDEIIEQISEFSKRVILADANLDKVLSAYNKHRDKPIREQAENIIHLFTLTNLGAPRAFKYFD TTIDRKVYRSTKEVLDATLIHQSITGLYETRIDLSQLGGD*

HF Cas9-NG (N497A, R661A, Q695A, Q926A/L111R, D1135V, G1218R, E1219F, A1322R, R1335V, T1337R/I)

MDKKYSIGLDIGTNSVGWAVITDEYKVPSKKFKVLGNTDRHSIKKNLIGALLFDSGETAEATRL KRTARRRYTRRKNRICYLQEIFSNEMAKVDDSFFHRLEESFLVEEDKKHERHPIFGNIVDEVA YHEKYPTIYHLRKKLVDSTDKADLRLIYLALAHMIKFRGHFLIEGDLNPDNSDVDKLFIQLVQT YNQLFEENPINASGVDAKAILSARLSKSRRLENLIAQLPGEKKNGLFGNLIALSLGLTPNFKSN 
FDLAEDAKLQLSKDTYDDDLDNLLAQIGDQYADLFLAAKNLSDAILLSDILRVNTEITKAPLSAS MIKRYDEHHQDLTLLKALVRQQLPEKYKEIFFDQSKNGYAGYIDGGASQEEFYKFIKPILEKM DGTEELLVKLNREDLLRKQRTFDNGSIPHQIHLGELHAILRRQEDFYPFLKDNREKIEKILTFRI PYYVGPLARGNSRFAWMTRKSEETITPWNFEEVVDKGASAQSFIERMTAFDKNLPNEKVLP KHSLLYEYFTVYNELTKVKYVTEGMRKPAFLSGEQKKAIVDLLFKTNRKVTVKQLKEDYFKKI ECFDSVEISGVEDRFNASLGTYHDLLKIIKDKDFLDNEENEDILEDIVLTLTLFEDREMIEERLK TYAHLFDDKVMKQLKRRRYTGWGALSRKLINGIRDKQSGKTILDFLKSDGFANRNFMALIHD DSLTFKEDIQKAQVSGQGDSLHEHIANLAGSPAIKKGILQTVKVVDELVKVMGRHKPENIVIE MARENQTTQKGQKNSRERMKRIEEGIKELGSQILKEHPVENTQLQNEKLYLYYLQNGRDMY VDQELDINRLSDYDVDHIVPQSFLKDDSIDNKVLTRSDKNRGKSDNVPSEEVVKKMKNYWR QLLNAKLITQRKFDNLTKAERGGLSELDKAGFIKRQLVETRAITKHVAQILDSRMNTKYDEND KLIREVKVITLKSKLVSDFRKDFQFYKVREINNYHHAHDAYLNAVVGTALIKKYPKLESEFVYG DYKVYDVRKMIAKSEQEIGKATAKYFFYSNIMNFFKTEITLANGEIRKRPLIETNGETGEIVWD KGRDFATVRKVLSMPQVNIVKKTEVQTGGFSKESIRPKRNSDKLIARKKDWDPKKYGGFVS PTVAYSVLVVAKVEKGKSKKLKSVKELLGITIMERSSFEKNPIDFLEAKGYKEVKKDLIIKLPKY SLFELENGRKRMLASARFLQKGNELALPSKYVNFLYLASHYEKLKGSPEDNEQKQLFVEQH KHYLDEIIEQISEFSKRVILADANLDKVLSAYNKHRDKPIREQAENIIHLFTLTNLGAPRAFKYFD TTIDRKVYRSTKEVLDATLIHQSITGLYETRIDLSQLGGD*

Cellular DNA polymerase domains

PolB

MSKRKAPQETLNGGITDMLTELANFEKNVSQAIHKYNAYRKAASVIAKYPHKIKSGAEAKKLP GVGTKIAEKIDEFLATGKLRKLEKIRQDDTSSSINFLTRVSGIGPSAARKFVDEGIKTLEDLRKN EDKLNHHQRIGLKYFGDFEKRIPREEMLQMQDIVLNEVKKVDSEYIATVCGSFRRGAESSGD MDVLLTHPSFTSESTKQPKLLHQVVEQLQKVHFITDTLSKGETKFMGVCQLPSKNDEKEYPH RRIDIRLIPKDQYYCGVLYFTGSDIFNKNMRAHALEKGFTINEYTIRPLGVTGVAGEPLPVDSE KDIFDYIQWKYREPKDRSE*

\section{PoID1}

MDGKRRPGPGPGVPPKRARGGLWDDDDAPRPSQFEEDLALMEEMEAEHRLQEQEEEELQ SVLEGVADGQVPPSAIDPRWLRPTPPALDPQTEPLIFQQLEIDHYVGPAQPVPGGPPPSHG SVPVLRAFGVTDEGFSVCCHIHGFAPYFYTPAPPGFGPEHMGDLQRELNLAISRDSRGGRE LTGPAVLAVELCSRESMFGYHGHGPSPFLRITVALPRLVAPARRLLEQGIRVAGLGTPSFAP YEANVDFEIRFMVDTDIVGCNWLELPAGKYALRLKEKATQCQLEADVLWSDVVSHPPEGPW QRIAPLRVLSFDIECAGRKGIFPEPERDPVIQICSLGLRWGEPEPFLRLALTLRPCAPILGAKV QSYEKEEDLLQAWSTFIRIMDPDVITGYNIQNFDLPYLISRAQTLKVQTFPFLGRVAGLCSNIR DSSFQSKQTGRRDTKVVSMVGRVQMDMLQVLLREYKLRSYTLNAVSFHFLGEQKEDVQHS IITDLQNGNDQTRRRLAVYCLKDAYLPLRLLERLMVLVNAVEMARVTGVPLSYLLSRGQQVK VVSQLLRQAMHEGLLMPVVKSEGGEDYTGATVIEPLKGYYDVPIATLDFSSLYPSIMMAHNL CYTTLLRPGTAQKLGLTEDQFIRTPTGDEFVKTSVRKGLLPQILENLLSARKRAKAELAKETD PLRRQVLDGRQLALKVSANSVYGFTGAQVGKLPCLEISQSVTGFGRQMIEKTKQLVESKYTV ENGYSTSAKVVYGDTDSVMCRFGVSSVAEAMALGREAADWVSGHFPSPIRLEFEKVYFPYL LISKKRYAGLLFSSRPDAHDRMDCKGLEAVRRDNCPLVANLVTASLRRLLIDRDPEGAVAHA QDVISDLLCNRIDISQLVITKELTRAASDYAGKQAHVELAERMRKRDPGSAPSLGDRVPYVIIS AAKGVAAYMKSEDPLFVLEHSLPIDTQYYLEQQLAKPLLRIFEPILGEGRAEAVLLRGDHTRC KTVLTGKVGGLLAFAKRRNCCIGCRTVLSHQGAVCEFCQPRESELYQKEVSHLNALEERFS RLWTQCQRCQGSLHEDVICTSRDCPIFYMRKKVRKDLEDQEQLLRRFGPPGPEAW*

\section{PolH}


MATGQDRVVALVDMDCFFVQVEQRQNPHLRNKPCAVVQYKSWKGGGIIAVSYEARAFGVT RSMWADDAKKLCPDLLLAQVRESRGKANLTKYREASVEVMEIMSRFAVIERASIDEAYVDLT SAVQERLQKLQGQPISADLLPSTYIEGLPQGPTTAEETVQKEGMRKQGLFQWLDSLQIDNLT SPDLQLTVGAVIVEEMRAAIERETGFQCSAGISHNKVLAKLACGLNKPNRQTLVSHGSVPQL FSQMPIRKIRSLGGKLGASVIEILGIEYMGELTQFTESQLQSHFGEKNGSWLYAMCRGIEHDP VKPRQLPKTIGCSKNFPGKTALATREQVQWWLLQLAQELEERLTKDRNDNDRVATQLVVSI RVQGDKRLSSLRRCCALTRYDAHKMSHDAFTVIKNCNTSGIQTEWSPPLTMLFLCATKFSAS APSSSTDITSFLSSDPSSLPKVPVTSSEAKTQGSGPAVTATKKATTSLESFFQKAAERQKVK EASLSSLTAPTQAPMSNSPSKPSLPFQTSQSTGTEPFFKQKSLLLKQKQLNNSSVSSPQQN PWSNCKALPNSLPTEYPGCVPVCEGVSKLEESSKATPAEMDLAHNSQSMHASSASKSVLE VTQKATPNPSLLAAEDQVPCEKCGSLVPVWDMPEHMDYHFALELQKSFLQPHSSNPQVVS AVSHQGKRNPKSPLACTNKRPRPEGMQTLESFFKPLTH*

\section{Poll}

MEKLGVEPEEEGGGDDDEEDAEAWAMELADVGAAASSQGVHDQVLPTPNASSRVIVHVDL DCFYAQVEMISNPELKDKPLGVQQKYLVVTCNYEARKLGVKKLMNVRDAKEKCPQLVLVNG EDLTRYREMSYKVTELLEEFSPVVERLGFDENFVDLTEMVEKRLQQLQSDELSAVTVSGHV YNNQSINLLDVLHIRLLVGSQIAAEMREAMYNQLGLTGCAGVASNKLLAKLVSGVFKPNQQT VLLPESCQHLIHSLNHIKEIPGIGYKTAKCLEALGINSVRDLQTFSPKILEKELGISVAQRIQKLS FGEDNSPVILSGPPQSFSEEDSFKKCSSEVEAKNKIEELLASLLNRVCQDGRKPHTVRLIIRR YSSEKHYGRESRQCPIPSHVIQKLGTGNYDVMTPMVDILMKLFRNMVNVKMPFHLTLLSVCF CNLKALNTAKKGLIDYYLMPSLSTTSRSGKHSFKMKDTHMEDFPKDKETNRDFLPSGRIEST RTRESPLDTTNFSKEKDINEFPLCSLPEGVDQEVFKQLPVDIQEEILSGKSREKFQGKGSVSC PLHASRGVLSFFSKKQMQDIPINPRDHLSSSKQVSSVSPCEPGTSGFNSSSSSYMSSQKDY SYYLDNRLKDERISQGPKEPQGFHFTNSNPAVSAFHSFPNLQSEQLFSRNHTTDSHKQTVA TDSHEGLTENREPDSVDEKITFPSDIDPQVFYELPEAVQKELLAEWKRAGSDFHIGHK*

\section{PolK}

MDSTKEKCDSYKDDLLLRMGLNDNKAGMEGLDKEKINKIIMEATKGSRFYGNELKKEKQVN QRIENMMQQKAQITSQQLRKAQLQVDRFAMELEQSRNLSNTIVHIDMDAFYAAVEMRDNPE LKDKPIAVGSMSMLSTSNYHARRFGVRAAMPGFIAKRLCPQLIIVPPNFDKYRAVSKEVKEIL ADYDPNFMAMSLDEAYLNITKHLEERQNWPEDKRRYFIKMGSSVENDNPGKEVNKLSEHER SISPLLFEESPSDVQPPGDPFQVNFEEQNNPQILQNSVVFGTSAQEVVKEIRFRIEQKTTLTA SAGIAPNTMLAKVCSDKNKPNGQYQILPNRQAVMDFIKDLPIRKVSGIGKVTEKMLKALGIITC TELYQQRALLSLLFSETSWHYFLHISLGLGSTHLTRDGERKSMSVERTFSEINKAEEQYSLC QELCSELAQDLQKERLKGRTVTIKLKNVNFEVKTRASTVSSVVSTAEEIFAIAKELLKTEIDAD FPHPLRLRLMGVRISSFPNEEDRKHQQRSIIGFLQAGNQALSATECTLEKTDKDKFVKPLEM SHKKSFFDKKRSERKWSHQDTFKCEAVNKQSFQTSQPFQVLKKKMNENLEISENSDDCQIL TCPVCFRAQGCISLEALNKHVDECLDGPSISENFKMFSCSHVSATKVNKKENVPASSLCEKQ DYEAHPKIKEISSVDCIALVDTIDNSSKAESIDALSNKHSKEECSSLPSKSFNIEHCHQNSSST VSLENEDVGSFRQEYRQPYLCEVKTGQALVCPVCNVEQKTSDLTLFNVHVDVCLNKSFIQEL RKDKFNPVNQPKESSRSTGSSSGVQKAVTRTKRPGLMTKYSTSKKIKPNNPKHTLDIFFK*

\section{REV1}

MRRGGWRKRAENDGWETWGGYMAAKVQKLEEQFRSDAAMQKDGTSSTIFSGVAIYVNGY TDPSAEELRKLMMLHGGQYHVYYSRSKTTHIIATNLPNAKIKELKGEKVIRPEWIVESIKAGRL LSYIPYQLYTKQSSVQKGLSFNPVCRPEDPLPGPSNIAKQLNNRVNHIVKKIETENEVKVNGM NSWNEEDENNDFSFVDLEQTSPGRKQNGIPHPRGSTAIFNGHTPSSNGALKTQDCLVPMV NSVASRLSPAFSQEEDKAEKSSTDFRDCTLQQLQQSTRNTDALRNPHRTNSFSLSPLHSNT KINGAHHSTVQGPSSTKSTSSVSTFSKAAPSVPSKPSDCNFISNFYSHSRLHHISMWKCELT 
EFVNTLQRQSNGIFPGREKLKKMKTGRSALVVTDTGDMSVLNSPRHQSCIMHVDMDCFFVS VGIRNRPDLKGKPVAVTSNRGTGRAPLRPGANPQLEWQYYQNKILKGKAADIPDSSLWENP DSAQANGIDSVLSRAEIASCSYEARQLGIKNGMFFGHAKQLCPNLQAVPYDFHAYKEVAQTL YETLASYTHNIEAVSCDEALVDITEILAETKLTPDEFANAVRMEIKDQTKCAASVGIGSNILLAR MATRKAKPDGQYHLKPEEVDDFIRGQLVTNLPGVGHSMESKLASLGIKTCGDLQYMTMAKL QKEFGPKTGQMLYRFCRGLDDRPVRTEKERKSVSAEINYGIRFTQPKEAEAFLLSLSEEIQR RLEATGMKGKRLTLKIMVRKPGAPVETAKFGGHGICDNIARTVTLDQATDNAKIIGKAMLNMF HTMKLNISDMRGVGIHVNQLVPTNLNPSTCPSRPSVQSSHFPSGSYSVRDVFQVQKAKKST EEEHKEVFRAAVDLEISSASRTCTFLPPFPAHLPTSPDTNKAESSGKWNGLHTPVSVQSRLN LSIEVPSPSQLDQSVLEALPPDLREQVEQVCAVQQAESHGDKKKEPVNGCNTGILPQPVGT VLLQIPEPQESNSDAGINLIALPAFSQVDPEVFAALPAELQRELKAAYDQRQRQGENSTHQQ SASASVPKNPLLHLKAAVKEKKRNKKKKTIGSPKRIQSPLNNKLLNSPAKTLPGACGSPQKLI DGFLKHEGPPAEKPLEELSASTSGVPGLSSLQSDPAGCVRPPAPNLAGAVEFNDVKTLLRE WITTISDPMEEDILQVVKYCTDLIEEKDLEKLDLVIKYMKRLMQQSVESVWNMAFDFILDNVQ VVLQQTYGSTLKVT*

\section{REV3L (catalytic domain)}

MGLSPLSTEPKTQKLSNKKGSNTDTLRRVLLTQAKNQFAAVNTPQKETSQIDGPSLNNTYGF KVSIQNLQEAKALHEIQNLTLISVELHARTRRDLEPDPEFDPICALFYCISSDTPLPDTEKTELT GVIVIDKDKTVFSQDIRYQTPLLIRSGITGLEVTYAADEKALFHEIANIIKRYDPDILLGYEIQMHS WGYLLQRAAALSIDLCRMISRVPDDKIENRFAAERDEYGSYTMSEINIVGRITLNLWRIMRNE VALTNYTFENVSFHVLHQRFPLFTFRVLSDWFDNKTDLYRWKMVDHYVSRVRGNLQMLEQ LDLIGKTSEMARLFGIQFLHVLTRGSQYRVESMMLRIAKPMNYIPVTPSVQQRSQMRAPQCV PLIMEPESRFYSNSVLVLDFQSLYPSIVIAYNYCFSTCLGHVENLGKYDEFKFGCTSLRVPPD LLYQVRHDITVSPNGVAFVKPSVRKGVLPRMLEEILKTRFMVKQSMKAYKQDRALSRMLDA RQLGLKLIANVTFGYTSANFSGRMPCIEVGDSIVHKARETLERAIKLVNDTKKWGARVVYGD TDSMFVLLKGATKEQSFKIGQEIAEAVTATNPKPVKLKFEKVYLPCVLQTKKRYVGYMYETLD QKDPVFDAKGIETVRRDSCPAVSKILERSLKLLFETRDISLIKQYVQRQCMKLLEGKASIQDFI FAKEYRGSFSYKPGACVPALELTRKMLTYDRRSEPQVGERVPYVIIYGTPGVPLIQLVRRPV EVLQDPTLRLNATYYITKQILPPLARIFSLIGIDVFSWYHELPRIHKATSSSRSEPEGRKGTISQ YFTTLHCPVCDDLTQHGICSKCRSQPQHVAVILNQEIRELERQQEQLVKICKNCTGCFDRHIP CVSLNCPVLFKLSRVNRELSKAPYLRQLLDQF*

\section{PoIN}

MENYEALVGFDLCNTPLSSVAQKIMSAMHSGDLVDSKTWGKSTETMEVINKSSVKYSVQLE DRKTQSPEKKDLKSLRSQTSRGSAKLSPQSFSVRLTDQLSADQKQKSISSLTLSSCLIPQYN QEASVLQKKGHKRKHFLMENINNENKGSINLKRKHITYNNLSEKTSKQMALEEDTDDAEGYL NSGNSGALKKHFCDIRHLDDWAKSQLIEMLKQAAALVITVMYTDGSTQLGADQTPVSSVRGI VVLVKRQAEGGHGCPDAPACGPVLEGFVSDDPCIYIQIEHSAIWDQEQEAHQQFARNVLFQ TMKCKCPVICFNAKDFVRIVLQFFGNDGSWKHVADFIGLDPRIAAWLIDPSDATPSFEDLVEK YCEKSITVKVNSTYGNSSRNIVNQNVRENLKTLYRLTMDLCSKLKDYGLWQLFRTLELPLIPIL AVMESHAIQVNKEEMEKTSALLGARLKELEQEAHFVAGERFLITSNNQLREILFGKLKLHLLS QRNSLPRTGLQKYPSTSEAVLNALRDLHPLPKIILEYRQVHKIKSTFVDGLLACMKKGSISST WNQTGTVTGRLSAKHPNIQGISKHPIQITTPKNFKGKEDKILTISPRAMFVSSKGHTFLAADFS QIELRILTHLSGDPELLKLFQESERDDVFSTLTSQWKDVPVEQVTHADREQTKKVVYAVVYG AGKERLAACLGVPIQEAAQFLESFLQKYKKIKDFARAAIAQCHQTGCVVSIMGRRRPLPRIHA HDQQLRAQAERQAVNFVVQGSAADLCKLAMIHVFTAVAASHTLTARLVAQIHDELLFEVEDP QIPECAALVRRTMESLEQVQALELQLQVPLKVSLSAGRSWGHLVPLQEAWGPPPGPCRTE SPSNSLAAPGSPASTQPPPLHFSPSFCL* 


\section{PolL}

MDPRGILKAFPKRQKIHADASSKVLAKIPRREEGEEAEEWLSSLRAHVVRTGIGRARAELFE KQIVQHGGQLCPAQGPGVTHIVVDEGMDYERALRLLRLPQLPPGAQLVKSAWLSLCLQERR LVDVAGFSIFIPSRYLDHPQPSKAEQDASIPPGTHEALLQTALSPPPPPTRPVSPPQKAKEAP NTQAQPISDDEASDGEETQVSAADLEALISGHYPTSLEGDCEPSPAPAVLDKWVCAQPSSQ KATNHNLHITEKLEVLAKAYSVQGDKWRALGYAKAINALKSFHKPVTSYQEACSIPGIGKRMA EKIIEILESGHLRKLDHISESVPVLELFSNIWGAGTKTAQMWYQQGFRSLEDIRSQASLTTQQ AIGLKHYSDFLERMPREEATEIEQTVQKAAQAFNSGLLCVACGSYRRGKATCGDVDVLITHP DGRSHRGIFSRLLDSLRQEGFLTDDLVSQEENGQQQKYLGVCRLPGPGRRHRRLDIIVVPY SEFACALLYFTGSAHFNRSMRALAKTKGMSLSEHALSTAVVRNTHGCKVGPGRVLPTPTEK DVFRLLGLPYREPAERDW*

\section{Poll $3 M^{31}$}

MVQIPQNPLILVDGSSYLYRAYHAFPPLTNSAGEPTGAMYGVLNMLRSLIMQYKPTHAAVVF DAKGKTFRDELFEHYKSHRPPMPDDLRAQIEPLHAMVKAMGLPLLAVSGVEADDVIGTLARE AEKAGRPVLISTGDKDMAQLVTPNITLINTMTNTILGPEEVVNKYGVPPELIIDFLALMGDSSD NIPGVPGVGEKTAQALLQGLGGLDTLYAEPEKIAGLSFRGAKTMAAKLEQNKEVAYLSYQLA TIKTDVELELTCEQLEVQQPAAEELLGLFKKYEFKRWTADVEAGKWLQAKGAKPAAKPQET SVADEAPEVTATVISYDNYVTILDEETLKAWIAKLEKAPVFAFDTETDSLDNISANLVGLSFAIE PGVAAYIPVAHDYLDAPDQISRERALELLKPLLEDEKALKVGQNLKYDRGILANYGIELRGIAF DTMLESYILNSVAGRHDMDSLAERWLKHKTITFEEIAGKGKNQLTFNQIALEEAGRYAAEDA DVTLQLHLKMWPDLQKHKGPLNVFENIEMPLVPVLSRIERNGVKIDPKVLHNHSEELTLRLAE LEKKAHEIAGEEFNLSSTKQLQTILFEKQGIKPLKKTPGGAPSTSEEVLEELALDYPLPKVILEY RGLAKLKSTYTDKLPLMINPKTGRVHTSYHQAVTATGRLSSTDPNLQNIPVRNEEGRRIRQA FIAPEDYVIVSADYSQIELRIMAHLSRDKGLLTAFAEGKDIHRATAAEVFGLPLETVTSEQRRS AKAINFGLIYGMSAFGLARQLNIPRKEAQKYMDLYFERYPGVLEYMERTRAQAKEQGYVETL DGRRLYLPDIKSSNGARRAAAERAAINAPMQGTAADIIKRAMIAVDAWLQAEQPRVRMIMQV HDELVFEVHKDDVDAVAKQIHQLMENCTRLDVPLLVEVGSGENWDQAH*

Editor Architectures

BE4B constructs

Promoter-BPNLS-[Deaminase]-32 amino acid linker-[Cas9 effector domain]-BPNLS-

Terminator

\section{C-terminal glycosylase constructs}

Promoter-BPNLS-[Deaminase]-32 amino acid linker-[Cas9 effector domain]-SGGS-

[Glycosylase variant]-BPNLS-Terminator

\section{Glycosylase architecture constructs}

N-terminal: Promoter-BPNLS-[Glycosylase variant]-SGGS-[Deaminase]-32 amino acid linker-[Cas9 effector domain]-SGGS linker-BPNLS-Terminator Internal: Promoter-BPNLS-[Deaminase]-32 amino acid linker-[Glycosylase variant]-32 amino acid linker-[Cas9 effector domain]-BPNLS-Terminator C-terminal: Promoter-BPNLS-[Deaminase]-32 amino acid linker-[Cas9 effector domain]SGGS linker- [Glycosylase variant]-BPNLS-Terminator

\section{Single fusion screen hit architecture constructs}

N-terminal: Promoter-BPNLS-[Screen Hit]-32 amino acid linker-[Deaminase]-32 amino acid linker-UdgX-[Cas9 effector domain]-BPNLS-Terminator 
C-terminal: Promoter-BPNLS-[Deaminase]-32 amino acid linker-UdgX-[Cas9 effector domain] 32 amino acid linker-[Screen Hit]-BPNLS-Terminator

\section{Dual fusion screen hit architecture constructs}

Dual N- N- terminal: Promoter-BPNLS-[Screen Hit]-32 amino acid linker-[Screen Hit]-32 amino acid linker-[Deaminase]-32 amino acid linker-UdgX-[Cas9 effector domain]-BPNLSTerminator $\mathrm{N}$ - and C- terminal: Promoter-BPNLS-[Screen Hit]-32 amino acid linker-[Deaminase]-32 amino acid linker-UdgX-[Cas9 effector domain] 32 amino acid linker-[Screen Hit]-BPNLSTerminator 


\section{Supplementary References}

1. Nishimasu, $\mathrm{H}$. et al. Engineered CRISPR-Cas9 nuclease with expanded targeting space. Science 361, 1259-1262 (2018).

2. Kurt, I.C. et al. CRISPR C-to-G base editors for inducing targeted DNA transversions in human cells. Nature Biotechnology 39, 41-46 (2020).

3. Zhao, D. et al. Glycosylase base editors enable C-to-A and C-to-G base changes. Nature Biotechnology 39, 35-40 (2020).

4. Chen, L. et al. Programmable C:G to G:C genome editing with CRISPR-Cas9-directed base excision repair proteins. Nature Communications 12 (2021).

5. Komor, A.C. et al. Improved base excision repair inhibition and bacteriophage $\mathrm{Mu}$ Gam protein yields C:G-to-T:A base editors with higher efficiency and product purity. Science Advances 3, eaao4774 (2017).

6. Komor, A.C., Kim, Y.B., Packer, M.S., Zuris, J.A. \& Liu, D.R. Programmable editing of a target base in genomic DNA without double-stranded DNA cleavage. Nature 533, 420-424 (2016).

7. Liu, D.R. \& Koblan, L.W. Cytosine to Guanine Base Editor. World Intellectual Property Organization (2018).

8. Arbab, M. et al. Determinants of Base Editing Outcomes from Target Library Analysis and Machine Learning. Cell 182, 463-480.e430 (2020).

9. Sancar, A. DNA Excision Repair. Annual Review of Biochemistry 65, 43-81 (1996).

10. Wood, R.D. DNA Repair in Eukaryotes. Annual Review of Biochemistry 65, 135-167 (1996).

11. Modrich, P. \& Lahue, R. Mismatch Repair in Replication Fidelity, Genetic Recombination, and Cancer Biology. Annual Review of Biochemistry 65, 101-133 (1996).

12. Choi, J.-Y., Lim, S., Kim, E.-J., Jo, A. \& Guengerich, F.P. Translesion Synthesis across Abasic Lesions by Human B-Family and Y-Family DNA Polymerases $\alpha, \delta, \eta, \mathrm{l}$, $\mathrm{K}$, and REV1. Journal of Molecular Biology 404, 34-44 (2010).

13. Lin, W. et al. The human REV1 gene codes for a DNA template-dependent dCMP transferase. Nucleic Acids Res 27, 4468-4475 (1999).

14. Prindle, M.J. \& and molecular, L.-L.A. DNA polymerase delta in DNA replication and genome maintenance. Environmental and molecular mutagenesis 53, 666-682 (2012).

15. Anzalone, A.V. et al. Search-and-replace genome editing without double-strand breaks or donor DNA. Nature 576, 149-157 (2019).

16. Anzalone, A.V., Koblan, L.W. \& Liu, D.R. Genome editing with CRISPR-Cas nucleases, base editors, transposases and prime editors. Nature Biotechnology 38, 824-844 (2020).

17. Rees, H.A. et al. Improving the DNA specificity and applicability of base editing through protein engineering and protein delivery. Nature Communications 8, 15790 (2017).

18. Kim, Y.B. et al. Increasing the genome-targeting scope and precision of base editing with engineered Cas9-cytidine deaminase fusions. Nature Biotechnology 35, 371-376 (2017).

19. Koblan, L.W. et al. Improving cytidine and adenine base editors by expression optimization and ancestral reconstruction. Nature Biotechnology 36, 843-846 (2018).

20. Gehrke, J.M. et al. An APOBEC3A-Cas9 base editor with minimized bystander and off-target activities. Nature Biotechnology 36, 977-982 (2018). 
21. Sang, P.B., Srinath, T., Patil, A.G., Woo, E.-J. \& Varshney, U. A unique uracil-DNA binding protein of the uracil DNA glycosylase superfamily. Nucleic Acids Res 43, 84528463 (2015).

22. Jinek, M. et al. RNA-programmed genome editing in human cells. eLife 2, e00471 (2013).

23. Jinek, M. et al. A Programmable Dual-RNA-Guided DNA Endonuclease in Adaptive Bacterial Immunity. Science 337, 816-821 (2012).

24. Gasiunas, G., Barrangou, R., Horvath, P. \& Siksnys, V. Cas9-crRNA ribonucleoprotein complex mediates specific DNA cleavage for adaptive immunity in bacteria. PNAS 109, E2579-E2586 (2012).

25. Mali, P. et al. RNA-Guided Human Genome Engineering via Cas9. Science 339, 823826 (2013).

26. Cong, L. et al. Multiplex Genome Engineering Using CRISPR/Cas Systems. Science 339, 819-823 (2013).

27. Kleinstiver, B.P. et al. High-fidelity CRISPR-Cas9 nucleases with no detectable genome-wide off-target effects. Nature 529, 490-495 (2016).

28. Slaymaker, I.M. et al. Rationally engineered Cas9 nucleases with improved specificity. Science 351, 84-88 (2015).

29. Chen, J.S. et al. Enhanced proofreading governs CRISPR-Cas9 targeting accuracy. Nature 550, 407-410 (2017).

30. Lee, J.K. et al. Directed evolution of CRISPR-Cas9 to increase its specificity. Nature Communications 9, 3048 (2018).

31. Camps, M., Naukkarinen, J., Johnson, B.P. \& Loeb, L.A. Targeted gene evolution in Escherichia coli using a highly error-prone DNA polymerase I. PNAS 100, 9727-9732 (2003). 